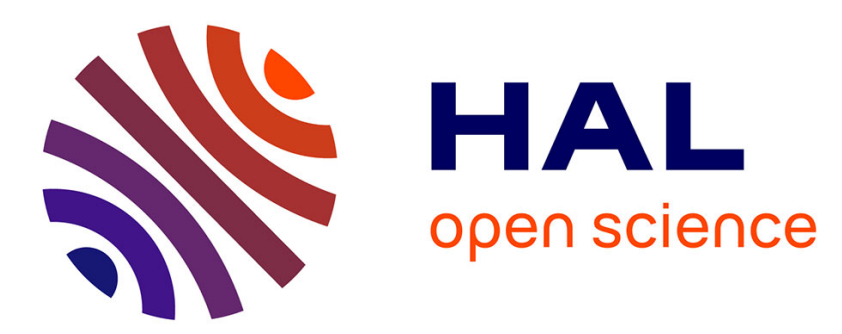

\title{
Philippe de La Hire, physicien : philosophie mécanique, mécanique rationnelle, mécanique appliquée
}

Christophe Schmit

\section{To cite this version:}

Christophe Schmit. Philippe de La Hire, physicien: philosophie mécanique, mécanique rationnelle, mécanique appliquée. Archives internationales d'histoire des sciences, 2020, 69 (182-183), pp.272-351. 10.1484/J.ARIHS.5.122117 . hal-03027681

\section{HAL Id: hal-03027681 \\ https://hal.science/hal-03027681}

Submitted on 27 Nov 2020

HAL is a multi-disciplinary open access archive for the deposit and dissemination of scientific research documents, whether they are published or not. The documents may come from teaching and research institutions in France or abroad, or from public or private research centers.
L'archive ouverte pluridisciplinaire HAL, est destinée au dépôt et à la diffusion de documents scientifiques de niveau recherche, publiés ou non, émanant des établissements d'enseignement et de recherche français ou étrangers, des laboratoires publics ou privés. 
Christophe Schmit, "Philippe de La Hire, physicien: philosophie mécanique, mécanique rationnelle, mécanique appliquée », Archives Internationales d'Histoire des Sciences, vol. 69, n 182-183, 2019 (2020), p. 272-351

\title{
Philippe de La Hire, physicien : philosophie mécanique, mécanique rationnelle, mécanique appliquée
}

\author{
Christophe Schmit \\ SYRTE, Observatoire de Paris, CNRS, Université PSL, Sorbonne Université, \\ 61 , avenue de l'Observatoire \\ F-75014 PARIS, France \\ christophe.schmit@obspm.fr
}

(33)140512203

\begin{abstract}
:
This article gives characteristics of Philippe de La Hire's mechanics. By "mechanics" we mean both mechanical philosophy and rational and applied mechanics, and for each we analyze La Hire's principles and methods. We show that his physics is in line with mechanical philosophy, especially that of Descartes, and he also seems to follow Gassendi and mechanistic chemists from the turn of the 17th and 18th centuries. Putting La Hire's rational mechanics in perspective with that of some members of the French Academy of science, we shows that he favours the "Ancients" over the "Moderns" according to his own terminology. Generally speaking, La Hire favours geometry and "synthesis" rather than analytical developments. In statics, the Archimedean axiomatic remains a model, and we also show that he seeks to perfect machines by applying statics and geometry to them but he doesn't propose tools quantifying their functioning and improvements, unlike some scholars of the time. Moreover, La Hire's kinematics and dynamics depend on the geometry of the problem studied and on the possibility of geometrically interpreting Galilean propositions. The concepts of dynamics and the procedures of infinitesimal geometry in La Hire's work echoed practices prior to the development of analytical mechanics.
\end{abstract}

Keywords :

Mechanical philosophy, Statics, Dynamics, Applied Mechanics, Principles, Methods, Geometry, Calculus

\section{Philippe de La Hire, physicist: mechanical philosophy, rational mechanics, applied mechanics}

\section{Résumé :}

Cet article donne les caractéristiques de la mécanique de Philippe de La Hire. Par «mécanique ", nous entendons à la fois la philosophie mécanique et la mécanique rationnelle et appliquée, et pour chacune nous analysons les principes et les méthodes de La Hire. Nous montrons que sa physique s'inscrit dans le cadre de la philosophie mécanique, en particulier celle de Descartes, et qu'il semble également suivre Gassendi et des chimistes mécanistes du tournant des XVII ${ }^{e}$ et XVIII ${ }^{e}$ siècles. En mettant en perspective la mécanique rationnelle de La Hire avec celle de certains membres de l'Académie royale des sciences de Paris, nous montrons qu'il privilégie les «Anciens » par rapport aux « Modernes » selon sa propre terminologie. D’une manière générale, La Hire privilégie la géométrie et la « synthèse » plutôt que les développements analytiques. En statique, l'axiomatique d'Archimède reste un modèle, et nous montrons aussi qu'il cherche à perfectionner les machines en leur appliquant la statique et la géométrie mais qu'il ne propose pas d'outils quantifiant leur fonctionnement et leurs améliorations, contrairement à certains savants de l'époque. De plus, la cinématique et la dynamique de La Hire dépendent de la géométrie du problème étudié et de la possibilité d'interpréter géométriquement les propositions galiléennes. Les concepts de dynamique et les procédures de la géométrie infinitésimale dans les travaux de La Hire font écho aux pratiques antérieures au développement de la mécanique analytique.

\section{Mots-clés :}

Statique, Dynamique, Mécanique appliquée, Principes, Méthodes, Géométrie, Calcul différentiel, Philosophie mécanique 


\section{Introduction}

Environ le tiers des exposés de Philippe de La Hire à l'Académie royale des sciences porte sur des questions de physique, et plus de la moitié de celles-ci sont soit strictement théoriques soit relèvent d'une science appliquée aux arts, autrement dit appartiennent à la catégorie "Physique (Théorie, Application)" définie dans l'Introduction de ce volume ${ }^{1}$. Parmi les 88 interventions académiques de La Hire dénombrées dans cette catégorie, 2 traitent d'acoustique, 17 d'optique et 69 de mécanique ${ }^{2}$; c'est dire la place importante qu'occupe cette dernière. Cet article se propose alors de dresser des caractéristiques essentielles de la physique de La Hire par l'examen des principes et des méthodes qu'il met en œuvre en mécanique en comparant notamment ses travaux à ceux de certains de ses confrères académiciens. Plus précisément, que regroupe-t-on ici sous l'appellation "mécanique" et quel est le corpus analysé ?

"Mécanique" s'emploie comme adjectif et peut-être associé à la "philosophie mécanique", synonyme de "mécanisme", laquelle explique les phénomènes naturels à partir de principes dits "mécaniques", à savoir aussi bien les corpuscules constitutifs de la matière que les lois qui régissent leurs mouvements. Bien que peu de recherches de La Hire ressortissent à un tel domaine ${ }^{3}$, nous l'évoquons ici pour deux raisons. D'une part, parce qu'adopter cette philosophie revêt un caractère fondamental puisque tous les phénomènes reposeraient sur ses principes. D’autre part, parce que nous verrons que ce "mécanisme" intervient à différentes périodes de la carrière de La Hire et ce jusque dans les années 1710 : il s'agirait donc pour lui d'un cadre théorique durable.

De par son étymologie, le mot "mécanique" renvoie à la science des machines, mais il prend aussi à partir de la deuxième moitié du $17^{\mathrm{c}}$ siècle le sens plus global de science du mouvement laquelle énonce des lois de la nature ${ }^{4}$. On distingue alors deux types de mécanique, l'une dite rationnelle et l'autre pratique dévolue aux arts ${ }^{5}$ l les 69 communications de La Hire appartiennent à chacune des $\operatorname{deux}^{6}$. En particulier, La Hire publie un long mémoire, le «Traité des Epicyclö̈des, et [...] leur usage dans les Méchaniques ${ }^{7} »$, et un livre, le Traité de mécanique, consacré à la théorie et d'après son sous-titre «à ce qui est nécessaire dans la pratique des Arts»; nous examinerons ces deux opus qui parmi les exposés académiques du savant occupent une place quantitativement

\footnotetext{
1 Nous renvoyons à cette Introduction qui contient ces données statistiques et dans laquelle nous regroupons la physique en "Physique (Théorie, Application)" et "Physique (Observations, Expériences, Pratique)" ; nous y renvoyons aussi en rappelant que les académiciens exposent leurs propres travaux devant leurs confrères.

2 Voir l'Introduction de ce volume. LA HIRE, Mémoires de mathématique et de physique, 1694 contient le "Traité des épicycloïdes » et la «Dissertation des differens accidens de la Vuë » qui totalisent ensemble plus de la moitié des interventions en optique : 2 interventions pour le premier traité (les 26 juin et 10 juillet 1688) et 10 pour le deuxième (en 1693, les 4 et 11 février, les 11 et 18 avril, les 2 et 23 mai, les 13 et 20 juin, le 8 août et, en 1694, le 4 septembre). Les 5 interventions restantes concernent des réfractions dans l'ellipse et l'hyperbole (4 décembre 1688), la lecture commentée d'un traité théorique (18 juillet 1696), de l'optique géométrique et la vision (16 et 20 mai 1699, 20 mars 1709). Celles en acoustique (le 23 février et le 8 mars 1692) portent sur l'« Explication des differences des sons de la Corde tenduë sur la Trompette marine » insérée dans LA HIRE, Mémoires de mathématique et de physique, 1694.

${ }^{3}$ L'« Explication des principaux effets de la Glace, et du Froid» dans LA HiRE, Mémoires de mathématique et de physique, 1694 contient des principes et des explications mécaniques, mais rien ne suggère qu'elle ait été lue à l'Académie. Par ailleurs, nous constaterons que des mémoires d'astronomie évoquent ponctuellement des principes du mécanisme.

${ }^{4}$ Pour une telle évolution, voir notamment GABBEY, "Newton’s Mathematical Principles», 1992 et GABBEY, "Between Ars and Philosophia Naturalis", 1993. Sur des définitions du mot "mécanique" dans des traités et dictionnaires des $17 \mathrm{e}$ et 18 siècles, voir SCHMIT, « Méchanique, statique, dynamique », 2014 et 2015.

${ }^{5}$ L'expression "mécanique rationnelle" et cette division figurent notamment dans la «Præfatio ad Lectorem » de Newton, Philosophice Naturalis Principia Mathematica, 1687 (non paginée) : "Mechanicam vero duplicem Veteres constituerunt: Rationalem qua per Demonstrationes accurate procedit, \& Practicam. Ad praticam spectant Artes omnes Manuales, a quibus utiq; Mechanica nomen mutuata est». Sur cette question, voir GABBEY, «Between Ars and Philosophia Naturalis », 1993. Notre usage de l'expression "mécanique rationnelle" reprend le sens donné par Newton dans cette préface.

${ }^{6}$ Nous n'avons pas trouvé de communications concernant la philosophie mécanique.

${ }^{7}$ Le mémoire figure dans LA HiRE, Mémoires de mathématique et de physique, 1694, p. 1-96.
} 
importante $^{8}$, opus auxquels, par ailleurs, il se réfère à d'autres moments de sa carrière 9 . Nous compléterons ces analyses par des mémoires académiques et des manuscrits tirés des Procèsverbaux de l'Académie qui paraissent représentatifs des recherches de La Hire ${ }^{10}$.

Dans un premier temps, nous soutiendrons que même si le corpus lié à la philosophie mécanique est limité, La Hire inscrit cependant sa physique dans ce cadre. Après avoir défini cette philosophie et en avoir rappelé des principes fondamentaux, nous constaterons que La Hire s'appuie sur ces derniers et qu'il s'inspire de Descartes, de Gassendi et de chimistes mécanistes tout en restant original. Au regard du statut d'"hypotheses", selon le mot de La Hire, que revêtent à ses yeux ces principes, nous interrogerons la valeur des explications du mécanisme pour le savant.

Nous montrerons ensuite que La Hire élabore la statique sur des principes et des méthodes des "Anciens", pour reprendre sa propre terminologie, et qu'il se positionne explicitement contre des "Modernes". Nous préciserons les sens de ces appellations et détaillerons les fondements qu’il donne à cette science ainsi que l'articulation entre un savoir théorique et son application aux arts. Nous avancerons notamment l'idée que La Hire perfectionne des machines sans mettre en place des outils théoriques ou expérimentaux permettant de quantifier leur fonctionnement et ces perfectionnements. En particulier, il ne recourt ni aux frottements solides ni à un antécédent du travail mécanique à l'œuvre chez certains de ses confrères académiciens.

Enfin, nous remarquerons que les contributions de La Hire en cinématique et en dynamique s’appuient essentiellement sur la géométrie infinitésimale au détriment du calcul différentiel et intégral leibnizien. La Hire participe aux recherches de la fin du $17^{\mathrm{e}}$ siècle sur la nature de courbes sur lesquelles se meuvent des corps soumis à des conditions particulières, mais sans pour sa part recourir au calcul différentiel à l'encontre duquel il se montre critique. Nous mettrons aussi en évidence que son analyse de ces questions est tributaire de la géométrie du problème et de la possibilité d'interpréter géométriquement des propositions de cinématique galiléenne. Enfin, nous constaterons que c'est sur cette cinématique que La Hire s'appuie pour formuler un concept de force, et que sa dynamique et les procédures mathématiques qu'il emploie font écho à des pratiques antérieures à l'essor d'une mécanique de type analytique.

\title{
2. Philosophie mécanique
}

\subsection{Aspects terminologiques}

Selon James E. McGuire «mechanical » signifie que :

\begin{abstract}
[the] nature is governed by immutable geometrical laws; contact action is the only mode of change ; first principles are to be integrated with the experimental investigations; regularities are to be explained in mathematical form ; that all phenomena arrive from matter in motion, or matter and motion ; that compound bodies are composed of vortices (Descartes), centers of force (Leibniz), or tiny bits of matter conceived as atoms or corpuscles; that changes in phenomena result from the way in which internal particles of motion alter their configurations; that the 'new science' [la philosophie mécanique] conceives nature dynamically in terms of motion, rather than statically in terms solely of the size and shape of internal particles ; that occult qualities are to be banished from explanations which must be based on sensory experience in terms of clear and distinct ideas; or that nature is to be conceived in analogy to the operations of mechanical activities [...] Thus, while they [les philosophes] all agreed that contact action was a necessary condition for a mechanical explanation, there was no settled agreement as to sufficient conditions ${ }^{11}$.
\end{abstract}

\footnotetext{
${ }^{8}$ Parmi les 69 interventions en mécanique, 51 sont réalisées avant 1699 et 25 concernent le Traité de mécanique. Le «Traité des épicycloïdes » ne donne lieu qu’à deux interventions (le 13 décembre 1692 et le 10 janvier 1693) sans savoir ce qui a été lu : il contient une partie strictement mathématique et une autre de mécanique appliquée.

${ }_{9}^{9}$ Voir en particulier des questions de statique des voûtes abordées dans LA HiRE, Traité de mécanique, 1695, p. 465-470, ainsi qu'à de l'Académie royale d'architecture en 1707 et 1711 et dans LA HIRE, "Sur la construction des voûtes ", 1714, p. 70-78. Sur ces questions, voir BECCHI, «Idées manuscrites, théories imprimées », 2013.

${ }^{10}$ Notons que nous ne possédons pas tous les manuscrits de ces 69 interventions.

${ }^{11}$ MCGuirE, « Boyle’s Conception of Nature », 1972, p. 523.
} 
Sophie Roux remarque que cette philosophie implique :

premièrement, l'idée que toute transformation naturelle résulte d'une redistribution des mouvements dans la matière ; en second lieu, l'idée que tout être naturel est une machine ${ }^{12}$.

A ces deux points, Alan Gabbey ajoute « the ideal of mathematizing the world picture », " the belief in necessary laws of nature and of motion», et "a theory in which the spiritual and the immaterial have been banished from the domain of investigation ${ }^{13}$. Pour Andrew Janiak, l'action des corps entre eux par le seul contact apparait comme une «norme» pour cette physique ${ }^{14}$.

L'expression «philosophie mécanique » apparait autour des années $1660^{15}$. Boyle utilise indifféremment " mechanical hypothesis or philosophy et "corpuscular philosophy » pour regrouper « the Atomical and Cartesian hypotheses » dont les explications des phénomènes naturels reposent sur le mouvement de corpuscules - «minutes bodies» ou «minutes particles » - de différentes formes. Il évoque indifféremment Gassendi et Descartes comme protagonistes d'une telle philosophie, en gommant ce qui pourrait distinguer leurs théories, en particulier leurs rapports respectifs à l'atomisme et au vide ${ }^{16}$. Par ailleurs, Boyle se voit lui-même identifié au $18^{\mathrm{e}}$ siècle à un philosophe mécaniste, notamment par Leibniz et Fontenelle, alors même qu'il s'appuie sur des corpuscules doués de certaines qualités pour expliquer des phénomènes chimiques qui sont alors non réductibles aux mouvements, aux chocs et aux formes prises par une matière passive ${ }^{17}$. On retrouve la définition de Boyle dans le Lexicon Technicum de Harris :

Mechanical Philosophy, is the same with the Corpuscular, which endeavours to explicate the Phenomena of Nature from Mechanical Principles ; i. e. from the Motion, Rest, Figure, Position, Magnitude, \&c. of the Minute Particles of Matter ${ }^{18}$.

Il semble qu'il faille attendre 1721 pour que de telles expressions et définitions apparaissent dans un dictionnaire continental ${ }^{19}$. Ainsi, le dictionnaire dit de Trévoux précise que :

la Philosophie méchanique est la même que la Philosophie corpusculaire, c'est-à-dire, celle qui explique tous les effets de la Nature par des principes de Méchanique, la figure, l'arrangement, la disposition, la grandeur ou la petitesse, le mouvement des parties qui composent les corps naturels ${ }^{20}$.

\footnotetext{
12 Roux, La philosophie mécanique, 1996, p. 32-33. L'auteur évoque aussi l'absence de «sympathie », d'« antipathie » et d'« action à distance».

13 GABBEY, « Newton, active powers », 2002, p. 337-338.

${ }^{14}$ JANIAK, Newton as Philosopher, 2008, p. 53.

15 Sur cette expression qui figure manifestement chez Henry More en 1659, mais qui est surtout pensée par Robert Boyle, voir ROUx, La philosophie mécanique, 1996, p. 19-26 et GARBER, « Remarks on the Pre-history », 2012, p. 3-26.

${ }^{16}$ BOYLE, Some Specimens of an Attempt, 1999-2000, p. 86-88. Sur cette élimination des différents courants distincts de la philosophie mécanique et leur regroupement autour de principes généraux chez Boyle, voir Roux, «La philosophie mécanique de Boyle », 2000, p. 119-133 ; GARBER, «Remarks on the Pre-history », 2012, p. 6-8.

17 Sur la construction de cette image de Boyle et la non réduction de la chimie au mécanisme, voir CLERICUZIO, «A redefinition of Boyle’s chemistry », 1990 et JOLY, « Chimie et mécanisme », 2008.

${ }^{18}$ HARRIS, Lexicon Technicum, 1704 (non paginé).

${ }^{19}$ On ne trouve pas d'entrées de ce type dans le Dictionnaire Universel de 1690 d'Antoine Furetière, dans Le Dictionnaire de l'Académie Françoise de 1694 et le Nouvean Dictionnaire de l'Académie Françoise de 1718, dans le Dictionnaire Universel de Henri Basnage de Beauval (1701, 1708), pas plus que dans le Dictionnaire Universel François et Latin de 1704. Cela ne signifie pas que ces expressions n'existent pas sur le continent. Ainsi, FONTENELLE, « Eloge de M. Guglielmini », 1712, p. 165 évoque des «fictions poëtiques» à propos de «raisonnemens de Chimie», auxquels il oppose «la saine Philosophie [...] qui a entrepris de réduire à la simple méchanique corpusculaire cette Chimie si mysterieuse ». Voir aussi FONTENELlE, «Eloge de M. Poli », 1716, p. 132 : «la Philosophie corpusculaire [...] celle qui n'admet que des idées claires, figures \& mouvements». Ces deux éloges sont mentionnés dans Roux, «La philosophie naturelle à l'époque de Le Nôtre », 2006. Selon l'auteur, les premières occurrences sur le continent de telles expressions figurent chez Leibniz dans les années 1670.

20 « Méchanique », Dictionnaire Universel François et Latin, 1721, t. IV, p. 273 a.
} 
L'Encyclopédie de Diderot et D'Alembert évoque « la Physique méchanique \& corpusculaire » dans des termes proches ${ }^{21}$, et l'entrée « Corpusculaire » stipule qu'une telle philosophie n'attribue rien d'autre aux corps «que ce qui est renfermé dans l'idée d'une chose impénétrable \& étendue, \& qui peut être conçu comme une de ses modifications, comme la grandeur, la divisibilité, la figure, la situation, le mouvement \& le repos » : les propriétés de la matière ne se déduisent que de ces «qualités » et non de "formes substantielles ", et les couleurs, la chaleur, le froid, les saveurs etc. des corps ne résultent que de la disposition des particules qui les composent; l'article mentionne notamment l'œuvre de Descartes pour illustrer ces « opinions » ${ }^{22}$. Pour sa part, l'entrée «Cartésianisme » livre un précis de la cosmogonie du philosophe qui fait naittre le monde d'une matière homogène qui remplit l'intégralité de l'espace car «l'étendue \& la matiere sont la même chose », et des mouvements et des chocs de celle-ci tous les deux régis par des lois divines ${ }^{23}$. "Cartésianisme » est par ailleurs un des renvois des articles « Matiere subtile » et « Ether » qui chez les :

Cartésiens non-seulement pénetre, mais encore remplit exactement [...] tous les vuides des corps, ensorte qu'il n'y a aucun espace dans l'Univers qui ne soit absolument plein ${ }^{24}$.

Cette catégorie historiographique et historique revêt donc de multiples formes ; la philosophie mécanique englobe divers courants comme l'exemplifient les philosophies naturelles de Boyle, Gassendi, Descartes et Leibniz pour ne s'en tenir ici qu'aux savants mentionnés ci-dessus. Quoi qu'il en soit, l'importance de Descartes et de Gassendi, l'absence de vide, l'usage de corpuscules dont les formes particulières, les mouvements et les chocs expliquent les phénomènes sont autant d'éléments qu'on rencontre dans l'œuvre de La Hire et qui nous permettent d'inscrire sa pratique dans le cadre de la philosophie mécanique. Ce qui suit examine l'éther, la nature des corpuscules et leurs usages dans les explications mécaniques de La Hire.

2.2 Ether et corpuscules dans l'œuvre de La Hire

\subsubsection{Ether}

La Hire mentionne l'éther notamment à l'occasion de travaux d'astronomie dans lesquels ce milieu n'est pas spécifiquement l'objet d'étude. Bien qu'évoqué ponctuellement, La Hire s'y réfère cependant jusque dans des mémoires publiés vers la fin de sa vie.

Il écrit que :

on entend ordinairement par le mot vuide un espace qui est vuide des particules de l'air, tel qu'il est sur la surface de la terre ; c'est aussi ce qu'on nomme Eter ou matiere subtile. Cette matiere subtile pénetre tous les corps \& même les plus durs, en passant au travers de tous leurs pores les plus déliés dans aucune difficulté. Il semble de-là que cette matiere doit être indifférente au mouvement des corps qui la traversent ${ }^{25}$.

La Hire évoque indifféremment le mouvement d'un corps « dans l’Eter ou dans le vuide ${ }^{26} »$. Il précise aussi concevoir l'éther «sans aucune pesanteur ${ }^{27}$ ».

Au sujet d'une éclipse lunaire, La Hire remarque que l'ombre de la Terre parait « quelquefois assez terminée, \& quelquefois fort confuse \& inégale », ce dernier cas de figure résultant de «l'inégalité de l'extrémité de l'Atmosphere » qui, «tantôt plus rare \& tantôt plus dense », donne « une ombre inégale » car elle détourne « un peu plus ou un peu moins les derniers rayons qui la rencontre »; ceci entraîne "une figure de l'ombre [qui] ne sera point terminée, comme si

${ }^{21}$ D’AlemberT, « Physique », Encyclopédie, 1765, p. 539a.

${ }^{22}$ FORMEY, « Corpusculaire », Encyclopédie, 1754, p. 269b-270b.

23 Pestre, D’Alembert, « Cartésianisme », Encyclopédie, 1752, p. 716a-726a.

${ }^{24}$ D'AlEmBERT, « Ether », Encyclopédie, 1756, p. 51 b.

${ }^{25}$ LA HIRE, « Remarques sur la cheute des corps », 1716, p. 337.

${ }^{26}$ Ibid., p. 344.

${ }^{27}$ LA HIRE, «Sur la hauteur de l'atmosphere », 1717, p. 64. 
l'Atmosphere étoit un corps fort différent de l'Ether ${ }^{28} »$. A contrario, l'éther ne produit pas de telles inégalités et il faut donc le voir comme le lieu de la régularité ; sans matières, il n'entraîne pas de modification de la direction d'un rayon lumineux et constitue ainsi un espace homogène : les rayons lumineux issus d'un astre se "détourn[ent] en venant de l'Eter» seulement lorsqu'ils pénètrent l'atmosphère ${ }^{29}$; la lumière n'est déviée qu'à cause de corps sensibles et hétérogènes à l'éther.

La Hire estime que l'anneau lumineux visible autour de la Lune lors d'une éclipse solaire totale observée à Londres en 1715 n'est pas dû à une atmosphère du satellite, et souligne que dans toutes les observations astronomiques effectuées «nous n'avons rien trouvé qui pût nous faire conjecturer qu'il y eût autour du corps de la Lune une matiére différente de celle de l'Eter ». On pourrait supposer cette atmosphère suffisamment rare pour ne pas modifier le cours des rayons des astres passant vers la Lune, et ça serait la raison pour laquelle les observations ne donnent pas "d'altération dans la figure de quelques Planetes [...] ni dans le mouvement de ces mêmes Planetes ou des Astres ». Mais, remarque La Hire, «s'il y avoit une Atmosphere, quelque rare qu'elle fût, il faudrait que ce fût un corps plus dense que l'Eter, puisqu'il pourroit nous renvoyer quelques rayons du Soleil, pour nous faire paroitre l'Anneau éclairé qu'on y remarque »; et « si c'est un corps, il faut que ces mêmes rayons y souffrent une réfraction, tant en entrant qu'en sortant, comme il arrive à l'Atmosphere de la Terre, \& c'est ce qu'on ne peut nier, \& ce qui est contre la supposition » d'une atmosphère rare ne causant pas de réfractions. La Hire dénonce donc une contradiction, l'atmosphère devrait être rare pour s'accorder avec les observations, mais en même temps il faudrait aussi la considérer dense car par hypothèse c'est elle qui rend cet anneau visible ${ }^{30}$. Le mot « dense » renvoie à la seule matière sensible ${ }^{31}:$ les observations indiquent une absence de réfraction donc de corps et par conséquent, selon La Hire, la présence du seul éther.

Ainsi, l'éther remplit tout l'espace et il est une matière dite "subtile" car il pénètre tous les corps. Il n'est pas pesant, autrement dit il diffère des corps sensibles, et l'absence de matière implique celle d'une résistance : c'est un milieu "indifférent" au mouvement des corps. C'est aussi manifestement un espace homogène où se propage la lumière. Il est dit "vuide" en ce sens qu'il est dépourvu de corps susceptibles de résister ou de causer une réfraction.

\subsubsection{La nature de l'air, de l'ean et des sels}

Dans un mémoire publié en 1704, La Hire suppose que :

l'air tel qu'est celui qui environne la terre, \& que nous appellons Atmosphére, est un corps pesant, \& que les
particules dont il est composé sont des ressorts, qui par leur nature, de quelque figure qu'on veuille les imaginer,
sont capables d'une très-grande extension, \& d'un resserrement ou d'une compression presqu'infinie par
accident, comme par un poids dont ils seroient chargés, \& que ces mêmes particules n'étant point accrochées
les unes aux autres, le corps qu'elles composent est un liquide, lequel est aussi transparent ${ }^{32}$.

En 1717, La Hire se réfère à «M. Mariotte dans son Essai de la Nature de l'Airr ${ }^{33}$ ». Mariotte et Boyle exemplifient ce contexte d'interprétations mécaniques de propriétés physiques de l'air découvertes au $17^{\mathrm{e}}$ siècle comme sa compressibilité ${ }^{34}$. Mariotte conçoit que «l'air est quelque chose de semblable à du cotton qui estant pressé, occupe un tres-petit espace, et qui peut s'estendre a un

\footnotetext{
${ }^{28}$ LA HIRE, « Observations de l'Eclipse de Lune », 1706, p. 355.

${ }^{29}$ LA HIRE, « Suite de l'examen de la ligne courbe », 1704, p. 183.

${ }^{30}$ LA HIRE, «Explication de l'anneau lumineux », 1718, p. 162-163.

${ }^{31}$ Notons que chez Descartes et des auteurs du premier 18 siècle tels que Joseph Privat de Molières et Laurent Béraud, l'éther est dit "dense" non pas au sens qu'il serait pesant mais parce qu'il occupe tout l'espace. Voir SCHMIT, «Les dissertations sur la calcination », 2017, p. 277-278 et p. 283-286.

32 LA HIRE, « Examen de la ligne courbe », 1704, p. 52-53.

${ }^{33}$ LA HiRE, « Sur la hauteur de l'atmosphere », 1717, p. 58.

${ }^{34}$ Sur les expériences pneumatiques chez Boyle et Mariotte et leurs conceptions de la nature de l'air, voir notamment ANSTEY, «Le ressort de l'air selon Boyle et Mariotte », 2009. Pour une analyse des expériences sur l'élasticité de l'air chez Boyle et au 17e siècle, voir WEBSTER, "The discovery of Boyle's law », 1965.
} 
beaucoup plus grand »; « comme le cotton peut se developer, \& occuper un bien plus grand espace qu'il n'occupe ordinairement, l'air tout de mesme developpe ses spires, \& les unes s'appuyant sur les autres, elles agissent en ressort ${ }^{35} »$. Pour sa part, Boyle considère l'air comme " a heap of little Bodies, lying one upon another, as may be resembled as Fleece of Wooll ${ }^{36} »$. Les «spires », le « ressort de l'air » et son action renvoient à un modèle mécaniste illustré par Fontenelle selon lequel « il faut concevoir l'Air comme composé d'une infinité de petites lames à ressort, soit spirale, soit de telle autre figure qu'on jugera plus convenable ${ }^{37} »$. En 1702, La Hire évoque :

l'effort du ressort de l'air comprimé » qui se dilate sous l'effet, par exemple, de l'inflammation de la poudre à canon, dilatation à l'origine de la projection du boulet ; il recourt à des ressorts proprement dits dont il examine les efforts pour illustrer le comportement de l'air dont « les particules [...] sont aussi des ressorts ${ }^{38}$.

C'est à Descartes que La Hire renvoie concernant la nature de l'eau dont il suppose « comme M. Descartes, que les particules [...] soient longues \& flexibles ${ }^{39} »$. Descartes suppose l'eau " composée de deux sortes de parties toutes longues \& unies, dont les unes sont molles \& pliantes, \& les autres roides \& inflexibles », et lorsqu'elles sont séparées les unes des autres, les premières forment l'eau douce aux parties flexibles « ainsi que des petites anguilles », les secondes les « sels » ${ }^{40}$. Lorsque La Hire évoque les particules des « sels », il les suppose « déliées, longues, roides \& aiguës » qui se «joignent» plus "aisément» avec les particules de l'eau qu'à d'autres particules, l'eau se joignant aussi plus «facilement» à ces sels qu'à d'autres corps ${ }^{41}$. Les opérations chimiques entre ces particules relèvent manifestement pour La Hire d'adéquations entre leurs figures, ainsi que de mouvements et de chocs comme nous le constaterons ci-dessous. Aussi, outre Descartes, les propos du savant évoquent aussi les mécanismes physico-chimiques du tournant des $17^{\mathrm{e}}$ et $18^{\mathrm{e}}$ siècles, ces particules « roides \& aiguës » pouvant faire notamment écho aux " pointes » des sels acides de Nicolas et Louis Lémery ou encore de Wilhelm Homberg ${ }^{42}$.

En effet, selon La Hire le tonnerre et les éclairs résultent de «matiéres [...] élevées de la terre jusqu'à la moyenne region de l'air » qui peuvent « s'enflammer » ou donner lieu à « une fermentation subite qui produit la chaleur $»^{43}$. Il mentionne alors un mémoire de Nicolas Lémery qui veut donner « une idée sensible » des éruptions volcaniques, des tremblements de terre, du tonnerre et des éclairs à l'aide «d'une Opération de Chymie » issue d'un mélange sous forme d'une pâte de fer et de soufre : il se produit une «fermentation», un «gonflement», un tremblement, un dégagement de chaleur voire de feu qui « procéde de la penétration \& du frotement violent que les pointes acides du soulfre font contre les parties du fer» en présence d'air, opérations qui produisent un «vent sulfureux ${ }^{44} »$. Ceci modélise les « feux souterrains » à l'œuvre dans la nature et les « vents » produits montent dans le ciel «ce qui fait la matiére \& la cause du Tonnerre»: ce «soulfre exalté, s'embarrasse dans les nues, \& y étant battu \& comprimé fortement, il acquiert un mouvement assez grand pour s'y enflâmer \& y former l'Eclair en fendant la nue, \& s'élançant avec une très-grande rapidité »; ce mouvement cause le « bruit du Tonnerre » car « ce vent sulfureux sortant violemment d'un lieu étroit où il étoit contraint, frappe l'air très-rudement »; le " nitre subtile » ou "salpêtre »

\footnotetext{
35 MARiotTe, Essays de physique. Second Essay, 1679, p. 171.

36 BOYLE, New Experiments Physico-Mechanical, 1999-2000, p. 141-301.

${ }^{37}$ FONTENELLE, «Sur une nouvelle proprieté de l'air», 1704, p. 2. Voir aussi FONTENELLE, « Sur les effets du ressort de l'Air », 1704, p. 9 pour l'évocation des «ressorts» des «masses d'air [qui] se dilatent \& se débandent tous ensemble »; ce texte de Fontenelle résume le mémoire non publié LA HIRE, «Explication des Effets du Ressort de l'air», 1702, p. 160v-171v.

${ }^{38}$ LA HIRE, «Explication des Effets du Ressort de l'air », 1702, f. 168v et f. 162v.

${ }^{39}$ LA HIRE, « Explication des principaux effets de la Glace », 1694, p. 97.

${ }^{40}$ Descartes, Principes, 1996, Partie IV, art. 35, 36 et 48 p. 219-220 et p. 227.

${ }^{41}$ LA HIRE, « Explication des principaux effets de la Glace », 1694, p. 97.

42 Sur la chimie et le mécanisme à cette période, voir notamment JolY, Descartes et la chimie, 2011, p. 175-215, Clericuzio, Principles and Corpuscles, 2000, p. 163-212, MeTZger, Les doctrines chimiques en France, 1923.

${ }^{43}$ LA HiRE, " Explication des Effets du Ressort de l'air », 1702, f. 170r.

${ }^{44}$ LEMERY, «Explication Physique \& Chymique des Feux souterrains », 1703, p. 101-105.
} 
répandu dans l'air « augmente la force de son mouvement $\&$ de son action ${ }^{45}$. La Hire écrit que la «fermentation subite » présentée par Lémery suffit " pour montrer que des matiéres liquides et mediocrement échauffées peuvent s'enflâmer facilement et produire un effet violent en dilatant les ressorts de l'air ${ }^{46} »$. Il suppose que le mélange « des matiéres de soufre et de salpétre et d'autres de même nature » dans l'air peuvent s'enflammer ; comme pour la poudre à canon dont l'inflammation permet la dilation de l'air, « l'air [...] se dilate par cette flamme subite » et s'il ne rencontre que de l'air contre lequel faire son effort, ceci produit un éclair sans bruit; s'il est proche de nuages comprenant des "petites particules d'eau glacée, l'opposition de ce feu avec l'eau froide fera un très grand bruit par les secousses violentes de l'air, comme il arrive quand on plonge un fer rouge dans l'eau ${ }^{47} »$. Enfin, ce «feu » peut pousser «les parties de l'air grossier» et en «bande $[\mathrm{r}]$ les ressorts avec assez de force pour lui résister et pour l'obliger de retourner en arriére » : le «feu » se « détourne » et forme " ces éclairs qui sont comme un trait de feu brisé en plusieurs parties ${ }^{48}$ ». La Hire ne donne pas ici le détail des opérations chimiques mais paraît faire siens les mécanismes de Lémery et ses conceptions des « sels ».

La Hire attribue une «figure » à ces « particules à ressort de l'air » pour justifier leur propriété et précise aussi qu'elles « peuvent se dilater dans l'éther » sans pour autant expliciter la manière dont ce dernier agit pour causer leur « ressort $^{49}$ ». Il conçoit manifestement l'air d'une manière que dénoncera notamment l'académicien Joseph Privat de Molières qui estime qu'un tel modèle ne justifie pas mécaniquement l'élasticité de ce milieu, car c'est transposer les propriétés du tout l'élasticité de l'air - au particulier - le « ressort» de ses parties -, sans donner la cause physique de ce « ressort $^{50} »$; Molières estime que l'air de ceux qu'il nomme « Cartésiens » est « une chimere ${ }^{51} »$. Il juge, tout d'abord, qu'un ressort finit au cours du temps par perdre son élasticité, puis que ce modèle mécanique ne rend pas compte d'autres propriétés de l'air, notamment son extrême «lubricité », à savoir «la facilité qu'ont ses parties à se séparer les unes des autres ${ }^{52}$ ", ce qui a manifestement à voir avec ce que La Hire nomme le caractère « liquide » de l'air qui vient de ce que ses parties ne s'accrochent $\mathrm{pas}^{53}$. En effet, sur ce dernier point, Molières, qui critique Descartes, estime impensable que l'air soit un assemblage de « parties branchuës, diversement entrelacées » et "prodigieusement bandées»: cet assemblage rend difficilement possible que ces "parties» puissent se « détacher si facilement les unes des autres, se mouvoir en tous sens avec une extrême liberté $^{54} »$ comme cela semble être le cas pour l'air ; une critique aussi valable à ses yeux à l'encontre

${ }^{45}$ Ibid., p. 105-106.

${ }^{46}$ LA HIRE, « Explication des Effets du Ressort de l'air », 1702, f. 170r.

${ }^{47}$ Ibid., f. 170v. Cette dernière image figure chez Lémery, «Explication Physique \& Chymique des Feux souterrains », 1703, p. 107. Lémery écrit que du soufre en rencontrant l'eau des nuages peut s'éteindre en faisant une « détonnation» comme « il arrive quand on jette dans de l'eau quelque matiére solide rougie au feu »; pour Lémery, « cette détonnation contribue peut-être à faire le bruit du Tonnerre ».

${ }^{48}$ LA HiRE, " Explication des Effets du Ressort de l'air », 1702, f. 170v.

${ }^{49}$ LA HIRE, «Sur la hauteur de l'atmosphere », 1717, p. 58.

${ }^{50}$ MOLIERES, Leçons de Phisique, 1736, t. 2, p. 144-145. Molières interprète ce « ressort » en concevant l'air composé de petits tourbillons de matière subtile dont les forces centrifuges s'équilibrent. Voir SCHMIT, «Les quatre prix académiques », 2017, p. 204-207.

51 Molieres, Leçons de Phisique, 1736, t. 2, p. 147. Remarquons que le « ressort» des corps reçoit une explication mécanique chez Descartes, la matière subtile tendant à suivre une circulation rectiligne dans les pores d'un corps fléchi fait recouvrer à ce dernier sa figure d'origine ; Descartes ne transpose donc pas au particulier les propriétés du tout et son explication du « ressort» vient de la circulation de la matière subtile dans laquelle les particules d'air se trouvent, voir DesCarTes, Principes, 1996, Partie IV, art. 46 et 47, p. 226-227.

52 Molieres, Leçons de Phisique, 1736, t. 2, p. 102.

${ }^{53}$ Sur une définition de la liquidité, voir MAIRAN, Dissertation sur la Glace, 1716, p. 5-6 et 1717, p. 8-10 : un « fluide en general est un corps dont les parties [...] ne sont pas liées ensemble, qui cede aisement au toucher, qui resiste peu à la division, \& qui se repand comme de lui-même»; «le mouvement des parties des liquides n'est pas visible, parce que ces parties sont trop petites pour être apperçuës, mais il n’est pas moins réel ».

${ }^{54}$ Molieres, Leçons de Phisique, 1736, t. 2, p. 146-147. DesCARTES, Principes, 1996, Partie IV, art. 33, p. 218, art. 45 et 46, p. 226 évoque les « parties [de l'air] presque toutes fort molles \& flexibles, ainsi que des petites plumes ou des bouts de cordes forts deliées ». L'air est « un amas des parties du troisiéme element, qui sont si deliées \& tellement destachées les unes des autres, qu'elles obeïssent à tous les mouvemens de la matiere du Ciel qui est parmy elles : ce qui est la 
de Mariotte ${ }^{55}$. Molières dénonce aussi la conception de l'eau de Descartes : il juge que ce dernier attribue une flexibilité aux particules d'eau sans en donner la cause mécanique ${ }^{56}$. Plus généralement, Molières critique ceux qu’ils nomment les "Chimistes Cartésiens » qui, selon lui, recourent à des corpuscules de «figures » particulières qu'ils ne justifient pas afin d'expliquer des propriétés physiques $^{57}$.

Ainsi, La Hire s'inspire explicitement de Descartes et semble faire siens des travaux de chimie de la fin du $17^{\mathrm{e}}$ siècle menés par des savants que certains qualifient de «Cartésiens »: La Hire appartiendrait alors à cette famille ${ }^{58}$. Mais La Hire est aussi dit «Gassendiste » dans l'Encyclopédie ${ }^{59}$. Ces appellations témoignent de la diversité des courants qui traversent l'œuvre de La Hire, comme l'illustre son interprétation du froid et du gel de l'eau.

\subsection{Explications mécaniques du gel de l'eau}

\subsubsection{Quelques interprétations du froid et de la formation de la glace au tournant des $17^{e}$ et $18^{e}$ siècles}

Lorsque dans l'Encyclopédie le physicien Etienne Hyacinthe de Ratte évoque ce que «les Philosophes ont imaginé» pour notamment rendre compte du gel de l'eau, il se réfère essentiellement à deux « systèmes ». Le premier est basé sur le « refroidissement » de l'eau liquide : la « chaleur» étant conçue comme une des «principales causes» de la « fluidité », le milieu devient solide si cette «chaleur » qui met en mouvement « ses parties» diminue «à un certain degré ». Le deuxième est basé sur l'introduction de «quelque chose de plus» dans l'eau qui cause sa solidification. Ratte mentionne notamment Descartes et les « Gassendistes » pour illustrer chacune de ces deux conceptions ${ }^{60}$. Nous donnons ci-dessous quelques conceptions du froid et de la glace afin de contextualiser les travaux de La Hire et d'en souligner l'originalité.

Selon Descartes, « le froid \& le chaud » s'interprètent par le mouvement des « petites parties des cors que nous touchons » qui sont « agitées plus ou moins fort que de coustume » par la " matiere subtile » ou «telle autre cause que ce puisse estre» et qui mettent en mouvement nos nerfs ; «lorsqu'elles les agitent moins fort, cela cause le sentiment de froideur » et inversement pour la chaleur $^{61}$. Descartes écrit ensuite qu'

il y a telle proportion entre la force de cete matiere subtile, \& la resistance des parties des autres corps, que, lorsqu'elle est autant agitée, \& qu'elle n'est pas plus subtile qu'elle a coustume d'estre en ces quartiers contre la terre, elle a la force d'agiter \& de faire mouvoir separement l'une de l'autre, \& mesme de plier la pluspart des petites parties de l'eau entre lesquelles elle se glisse, \& ainsi de la rendre liquide ; mais [...] lorsqu'elle n'est pas plus agitée, ny moins subtile, qu'elle a coustume d'estre en ces quartiers au haut de l'air, ou qu'elle y est

cause qu'il est... rare, liquide \& transparent, \& que les petites parties dont il est composé, peuvent estre de toutes sortes de figures »; les corpuscules du troisième élément qui composent l'air se répartissent en trois espèces dont la première est constituée de « figures fort empeschantes..., \& dont les extremitez s'estendent diversement çà \& là, ainsi que des branches d'arbres ou choses semblables ».

55 Molieres, Leçons de Phisique, 1736, t. 2, p. 120.

56 Ibid., p. 243-246.

57 Voir MOLIERES, Leçons de phisique, 1737, t. 3, p. 128-140 pour ses critiques contre une chimie corpusculaire, et SCHMIT, La philosophie naturelle de Malebranche au XVIII e siècle, 2020 pour leur analyse.

${ }^{58}$ Soulignons que Molières rassemble sous une même appellation des savants aussi différents que Descartes, N. et L. Lémery, Boyle ou encore Homberg. Pour un examen de leurs différences et cette terminologie « cartésiens » appliquée en chimie, voir CLERICUZIO, Principles and Corpuscles, 2000, p. 163-212, JOLY, "Chimie et mécanisme dans la nouvelle Académie ", 2008, JOLY, Descartes et la chimie, 2011, Schmit, La philosophie naturelle de Malebranche au XVIII siècle, 2020.

${ }^{59}$ RATTE, « Glace », Encyclopédie, 1757, t. VII, p. 679b : « Les Gassendistes supposent [...] des corpuscules frigorifiques salins ou nitreux, qui s'introduisant entre les pores d'un fluide, arrêtent le mouvement de ses parties, \& les fixent en un corps solide \& dur. Cette opinion a été adoptée par le célebre M. de la Hire».

60 Ibid. Pour RatTe, «Froid», Encyclopédie, 1757, t. VII, p. 313a, la chaleur s'oppose à la « force de cohésion» des particules qui composent des corps et les rendent solides. Dans ce dernier article, Ratte remarque que « le plus grand nombre » de physiciens pense que le feu est « un corps particulier distingué de tous les autres » et que "ce même feu mis en mouvement, constitue la chaleur $»$.

${ }^{61}$ DESCARTES, Les météores, 1996, p. 236-237. 
quelquefois en hyver contre la terre, elle n'a point assés de force pour les plier \& agiter en cete façon, ce qui est cause qu'elles s'arestent confusement iointes \& posées l'une sur l'autre, \& ainsi [...] elles composent un cors dur, a savoir de la glace ${ }^{62}$.

De même, pour Rohault, si le mouvement de «la matiere du premier \& du second Element » était «fort ralenty», ou si elle était «extraordinairement subtile», alors elle n'entretiendrait plus la liquidité et transformerait l'eau en glace ${ }^{63}$. Suivant Régis les «particules d'Eau » ont le mouvement que la «Matiere subtile » leur donne, et «il faut de nécessité que l'Eau soit d'autant plus liquide que la Matiere subtile est plus agitée, \& qu'au contraire l'Eau approche d'autant plus de la nature de la glace que la matiere subtile a moins d'agitation ${ }^{64} »$.

On retrouve le mouvement et la "subtilité" du milieu à l'origine de la chaleur et du froid par l'agitation de corpuscules chez Mariotte et Mairan, dont les publications se situent au commencement et à la fin de la carrière académique de La Hire, savants qui ne suivent pas précisément les mécanismes de Descartes ${ }^{65}$. Mariotte conçoit que les «qualités » qui paraissent contraires aux « qualités actives » ne sont en fait qu'une « privation » de celles-ci, comme l'obscurité l'est de la lumière et le repos du mouvement. Ce qui est contraire à la chaleur, à savoir le froid, « doit suivre la mesme regle ». Ainsi :

d'autant que le mouvement est le seul principe, ou du moins un des principes de la chaleur [...] si le mouvement a pour son contraire le repos, qui est une privation, le contraire de la chaleur qui est le froid, sera aussi une privation, \& si les corps ne sont chauds que par un mouvement violent de leurs particules, il s'ensuit nécessairement que lorsque leur mouvement cesse, ils demeurent froids \& sans chaleur ${ }^{66}$.

Mariotte estime que « la fluïdité des liqueurs aqueuses » vient du mouvement de « leurs parties » agitées par le mouvement d'une «matiére aërienne », distincte de l'éther, mouvement « entretenu par la chaleur ». Aussi, «lorsqu'il fait un très grand froid, ce mouvement devient si foible qu'il ne peut plus agiter les parties de l'eau, de maniére qu'elles s'attachent au vaisseau; \& puis elles se joignent les unes aux autres ; \& de là viennent les filets \& ces lames de glaces que l'on voit paroître lorsque l'eau commence à geler ${ }^{67} \gg$.

Pour sa part, Mairan conçoit l'éther composé de petits tourbillons de matière subtile qui s'équilibrent mutuellement par leurs forces centrifuges, et ce à l'instar de Malebranche, ces tourbillons expliquant notamment la cohésion des corps par la pression qu'ils exercent sur ceux$\mathrm{ci}^{68}$. Les «parties » d'un liquide « nagent», « glissent » dans l'éther et le « plus ou le moins de cette matiere [subtile] enfermée dans un liquide, selon qu'elle a plus ou moins d'agitation \& de ressort [...] fait principalement le plus ou le moins de liquidité $»^{69}$. Cette mobilité n'entraîne pas la dissipation du liquide car il y a un équilibre global entre ses «parties » que la matière subtile anime,

${ }^{62} \mathrm{Ibid}$. La matière subtile se meut plus vite vers la surface de la terre que dans l'air. Par ailleurs, elle est composée de parties de grandeurs différentes et « moins cette matiere est subtile, c'est-à-dire composée de parties moins petites, plus elle peut agiter les parties des autres cors », autrement dit plus elle a de « force ». Ibid., p. 234-235.

${ }^{63}$ ROHault, Traité de physique, 1671, p. 165.

${ }^{64}$ REGIS, Systême de philosophie, 1691, t. 3, p. 240-241.

${ }^{65}$ Mariotte et La Hire mènent des travaux en commun et rendent notamment compte à l'Académie d'observations et d'expériences d'optique (11 mars et 6 mai 1679), voir PICOLET, « Annexe IV. La Hire entre sciences et architecture », 2013, p. 263-264.

${ }^{66}$ MARIOTTE, Essays de physique. Troisieme Essay, 1679, p. 17-20.

${ }^{67}$ MARIOTTE, Expériences touchant les couleurs et la congélation de l'eau, 1717, p. 606. Cette « matiére aërienne » remplie l'eau. Elle donne naissance aux « bulles » lorsqu'on la fait bouillir, ou quand on la place un récipient d'eau dans une machine pneumatique ; Mariotte écrit « qu'elle se change en air ». Cette «matiére » est de l'air dissout et condensé dans l'eau. Voir MARIOTTE, Essays de physique. Second Essay, 1679, p. 107.

${ }^{68}$ MAIRAN, Dissertation sur la Glace, 1716, p. 8-10. Dans la quatrième édition de cet ouvrage, MAIRAN, Dissertation sur la Glace, 1749, p. xxvj et p. xvj-xvij écrit que « dès qu'on voudra attacher une idée claire \& distincte à ce fluide [la matière subtile], on tombera nécessairement dans l'hypothèse des petits tourbillons dont le P. Malebranche a composé sa matière éthérée » et rejette la matière subtile de Descartes. Sur Malebranche et cette théorie au $17^{e}$ siècle, voir SCHMIT, La philosophie naturelle de Malebranche au XVIII siècle, 2020.

${ }^{69}$ MAIRAN, Dissertation sur la Glace, 1716, p. 10-11. 
et l'éther à l'intérieur et à l'extérieur de ce liquide ${ }^{70}$. Pour obtenir de la glace, il faut alors selon Mairan «chasse[r] » ou «diminue[r] [le] mouvement ou [le] ressort» de l'éther situé dans les interstices de l'eau, et pour liquéfier il faut introduire « une quantité suffisante de matiere subtile dans [les] pores [de l'eau], ou augmente[r] assez le mouvement ou le ressort de celle qui s'y trouve enfermée, pour qu'elle puisse separer les parties qui s'unissent par leur surface». La chaleur provient du mouvement de l'éther communiqué aux corps, et le froid entraîne un moindre mouvement des tourbillons à l'origine d'une rupture de leur équilibre. Si un lieu se refroidit, la mobilité de la matière subtile extérieure à un récipient contenant un liquide diminue et "par consequent elle ne sçauroit se trouver en équilibre avec celle qui est dans le liquide»: moins comprimé du dehors, « elle doit s'échapper du côté où elle trouve moins de resistance que dans le liquide, c'est-à-dire vers les extremitez $\&$ hors du liquide ». Cette « effusion » continue « jusqu'à ce que le nombre, la tension \& la vitesse des molécules » diminuent « pour demeurer en équilibre avec la matiere subtile du dehors »; les «parties » solides du liquide perdent alors de leur mouvement et se rapprochent; elles "s'acrocheront ou s'entrelasseront, si elles sont crochües ou rameuses», l'intensification du froid augmentant ce processus qui conduit à la formation d'un corps solide ${ }^{71}$.

Contrairement à ces auteurs, Gassendi ne fait intervenir ni matière subtile, ni «matiére aërienne ». La chaleur provient d'« atomes calorifiques» qui «doüez de telle grandeur, de telle figure, \& de tel mouvement, s'insinuent, penetrent, remuent, \& separent, \& produisent les autres effets qu'on rapporte ordinairement à la chaleur». Ces atomes peuvent «entrer» dans les corps, " remuer", "d'inciser», « resoudre », et les corps qui les contiennent et qui peuvent les « envoyer en dehors » sont « estimez chauds ${ }^{72} »$. Ces atomes sont «tres petis », « spheriques, parce qu'ils se meuvent tres-facilement, \& qu'ils s'insinuent de tous costez », «tres rapides, parce que par la rapidité de leur mouvement ils choquent avec vehemence, ebranlent, ecartent, \& dissolvent ${ }^{73} »$. Il existe aussi des « atomes de froideur, ou atomes frigorifiques » qui, par opposition aux précédents, sont capables « d'assembler, \& de resserrer ». Comme le froid est opposé à la chaleur, la forme des «atomes de froideur» se construit aussi en opposition aux « atomes calorifiques » et Gassendi les considère « Pyramidaux ${ }^{74} »$.

En rapprochant des mains gelées du feu on sent « une certaine douleur aiguë » car « les atomes de chaleur» qui entrent dans les pores «poussent \& repoussent diversement les atomes de froideur » qui les occupent, et « il arrive que ces derniers sont contraints de se tourner pour sortir, \& [...] picquent diversement, percent, \& dechirent l'organe du sens, tant par leurs pointes que par leurs costez tranchants ». Aussi, avant d'approcher des membres gelés du feu, il faut les mettre dans l'eau froide ou la neige pour éviter qu'ils « ne se corrompent ou ne se pourrissent ». Il en est de même pour des fruits gelés. Ainsi, la gelée autour d'une pomme tient ses " pores fortement, \& etroitement serrez », et une fois dissoute par l'eau ou la neige dans laquelle on la place, les «atomes

\footnotetext{
${ }^{70}$ Ibid., p. 17-18. L'équilibre est issu d'une égalité entre les forces centrifuges. L'éther dans le liquide possède moins de vitesse que celui du dehors puisqu'il y rencontre des obstacles à son mouvement, « obstacles d'autant plus considerables que la densité du liquide est plus grande, que ses parties [...] sont plus grosses, qu'elles ont plus de surface, \& que ces surfaces sont moins glissantes ». Mais ce qu'il perd en vitesse « est compensé par une plus grande tension du ressort de ses [au liquide] molecules, lequel augmente sa force à mesure qu’il est plus comprimé ».

${ }^{71}$ Ibid., p. 19-24.

${ }_{72}$ Bernier, Abregé de la philosophie de Gassendi, 1678, t. II, p. 77-78. De manière générale, les «atomes » sont «les principes substantiels mesmes ausquels tout mouvement, \& par consequent toute action doit estre attribuée ». Dans les paragraphes consacrés à Gassendi, nous suivrons ici la traduction de Bernier de Gassendi, Opera omnia, 1658, t. I, Sect. I, Livre VI, Chap. VI « De calore, \& Frigore », p. 394-401.

${ }^{73}$ Bernier, Abregé de la philosophie de Gassendi, 1678, t. II, p. 79-80.

${ }^{74}$ Ibid., p. 98-100. À l'égard de « la grandeur de la masse », « les atomes pyramidaux peuvent estre plus grands que les spheriques de toutes leurs pointes »; concernant la «figure », « il n’y en a aucune qui soit plus opposée à la spherique que la pyramidale, en ce qu'elle a des angles, \& qu'elle s'eloigne plus qu'aucune autre de cette infinité de petis costez ou petites faces insensibles qui peuvent estre considerées dans la sphere »; concernant le mouvement, «il n'y a aucun corps qui ait plus d'inhabilité au mouvement que le pyramidal». Il est précisé que « cette inhabilité ne se doit [...] pas considerer en plein vuide où tous les atomes sont egalement vistes, mais entant qu'ils sont embarrassez dans les composez, \& que par leurs mouvemens instestins ils font effort pour se degager ».
} 
de chaleur» du fruit qui sont dans un «mouvement, \& dans une espece d'effort perpetuel» trouvent « les passages plus libres, \& à demi-débouchez » et « poussent [...] dehors par ces mesmes passages les atomes de froideur » lesquels gèlent cette eau et font cette " petite glace mince » autour de la pomme gelée. Par ailleurs, « les corps de froideur» de l'eau et la neige «attirent en quelque maniere » ceux dans les corps gelés car il y a entre eux « une certaine sympathie fondée sur la ressemblance, \& convenance, ou conformité reciproque de leurs figures, \& consequemment de leurs mouvemens ${ }^{75} »$.

$\mathrm{La}$ «face » des « atomes frigorifiques » permet de « pousser » et « resserrer » des particules telles que celles de l'eau : «ces faces [triangulaires dues à la forme pyramidale de ces atomes] peuvent d'autant plus presser, \& arrester les corps, qu'elles se touchent par un plus grand nombre de parties, $\&[\ldots]$ plus elles sont embarrassées avec leurs petits angles, plus il leur est difficile de se debarasser, $\&$ plus fortement elles demeurent adherantes \& attachées; d'où vient que les petis corps qui ont de ces sortes de faces \& angles sont d'eux-mesmes astringens, \& que se fourrants d'ailleurs entre les parties des corps fluides, ils les rendent fixes, compactes $\&$ solides $^{76} »$. Ainsi, pour la formation de la glace, « il doit survenir des corps etrangers, tels que sont principalement les sels de Nitre, qui resserrant les corpuscules d'eau, luy ostent sa fluidité, \& la rendent ferme $\&$ solide $^{77} \gg$.

\subsubsection{Le froid et le gel de l'eau selon La Hire}

La Hire « suppose » que le « sentiment de froid» vient du moindre mouvement des « particules de l'eau » qui nous environnent par rapport à celles de « l'eau qui est au dedans des parties de nostre corps qu'elles touchent»; le froid parait plus grand à proportion que le mouvement des particules extérieures est moindre. Il fait alors l'«hypothese » que seuls «certains sels» sont capables «d'arrester le mouvement des particules de l'eau». Les «particules» de ces sels sont « déliées, longues, roides \& aiguës », et donc capables de pénétrer les métaux et le verre, et elles sont « emportées facilement dans l'air par son mouvement»; «les particules de l'eau s'arrestent plus facilement à ces sels qu'à tout autre corps, sans en excepter les autres sels, \& réciproquement [...] ces sels se joignent plus aisément aux particules de l'eau, qu'à celles des autres corps ». En supposant, "comme M. Descartes », les particules d'eau «longues \& flexibles », elles pourront perdre leurs mouvements en "s'entortillant» autour de ces sels, ne possédant alors que les mouvements de ceux-ci, et en perdant leur mouvement elles cesseront de composer un «corps fluide ${ }^{78} »$.

En pénétrant dans l'eau, les sels arrêtent le mouvement de quelques-unes de ses particules, et " plusieurs de ces particules de sel \& d'eau jointes ensemble venant à s'assembler les unes avec les autres, forment des filets glacez par où l'eau commence à se geler ». La Hire précise que "ces premieres parties de glace doivent retenir la figure des parties du sel qui leur sert de principe, comme il arrive dans la formation des mixtes » d'où, probablement, ces « filets » dus aux formes " déliées, longues, roides \& aiguës » des sels. Les particules de glace ainsi formées dans l'eau tiennent plus de place que celles d'eau dont elles sont issues à cause de l'ajout des sels mais aussi surtout par «les differens assemblages de ses particules gelées qui étant devenuës roides \& ne pouvant plus s'accommoder les unes aux autres, forment des vuides par l'effort qu'elles font en se liant ensemble, \& en dilatant considérablement les espaces d'air qui sont mélez parmi les particules de l'eau ». La Hire propose une analogie mécanique pour comprendre comment se font ces dilatations : « une certaine quantité de petites aiguilles couchées suivant leur longueur les unes sur les autres, occuperont bien moins de place, que si une partie de ces aiguilles estoit entortillée autour des

\footnotetext{
${ }^{75}$ Ibid., p. 100-102.

${ }^{76}$ Ibid., p. 103.

${ }^{77}$ Ibid., p. 214. Ce n'est donc pas dans la «nature» de l'eau, de l'air, de la terre, des «mixtes » qu'il faut chercher «la qualité de froid» mais «dans la nature de ces petis corps tels que sont ceux de Nitre, ou autres semblables que nous pouvons appeller esprits frigorifiques » qui les rendent froids en s'introduisant dans leurs pores, ibid., p. 109.

${ }^{78}$ LA HIRE, « Explication des principaux effets de la Glace, », 1694, p. 97.
} 
autres ». Chaque assemblage de filets de glaces étant plus léger que le volume d'eau dont il occupe la place $^{79}$, «ces petites parties de glaces [...] s'éleve vers la superficie ». Lorsque cette dernière en est couverte, les particules de sels qui viennent encore dans l'eau « lient \& attachent tous ces filets ensemble, [et] il se forme une crouste de glace qui renferme toute l'eau »; le processus se poursuit, les sels qui sont capables de passer à travers cette glace entrent en contact avec l'eau en-dessous de la surface et forment d'autres filets qui, à leur tour, s'élèvent et font " un effort contre l'eau dans laquelle ils nagent \& contre ce qui la contient», effort par lequel l'eau peut rompre la croûte de glace par endroits. Ces petites particules de glace peuvent se briser mutuellement par leurs efforts, et « les fragmens avec le peu d'eau qui reste s'attachant ensemble, \& à toute la glace qui s'est formée, tant autour du vaisseau [contenant l'eau], qu'au dessus, laissent dans le milieu un espace vuide assez grand, qui est la bulle d'air qu'on y voit ordinairement ${ }^{80} »$.

Le mécanisme de formation de la glace est suivi d'une série d'interprétations de phénomènes à partir de l'« hypothese » posée par La Hire qui ont pour fonction de la justifier.

Lorsqu'il y a plus de particules d'eau que de sels, l'eau se refroidit sans geler, car les particules d'eau arrêtées autour des sels ne peuvent pas se joindre: «il se rencontre entre deux trop de particules d'eau qui détachent celles qui sont déjà jointes aux particules de sels, en les choquant continuellement ; \& la froideur de l'eau augmente seulement par la privation du mouvement de quelques-unes de ses parties ». Par ailleurs, La Hire considère l'eau dans son "état naturel sans aucun mouvement \& liquide tout ensemble, c'est-à-dire que toutes les parties puissent estre dérangées par une force aussi petite qu'on la voudra supposer; ce qui est possible, si ces parties sont infiniment polies »; dans cet état l'eau ferait une impression de froid comme la glace si elle était capable d'arrêter le mouvement des particules d'eau dans la peau, mais comme ses parties sont disposées «à se mouvoir tres-facilement » elles ne font que peu d'« impression » sur nos «sens »; a contrario les particules d'eau glacée nécessitent un effort pour être mises en mouvement ${ }^{81}$.

Selon La Hire, « les corps gras \& huileux, \& qui n'ont que tres-peu de parties d'eau, ne sont pas propres à retenir ces sels, \& par conséquent ils ne se gelent pas facilement »; c'est le cas du vin, des fruits, des huiles etc. Si on place un fruit gelé dans une eau " pas trop chaude », toutes les particules des sels « arrestées [...] en partie » aux « particules d'eau » du fruit «s'en détachent facilement pour s'insinuer dans l'eau qui environne le fruit, parce qu'elles trouvent plus de facilité à se joindre à ces parties d'eau, qu'à celle qui sont dans le fruit entremélées de parties huileuses ; ainsi il arrive que le fruit se dégele presque tout d'un coup, \& qu'il se fait tout autour une crouste de glace fort dure \& fort claire ». Si l'eau est trop chaude, ses particules ont un trop grand mouvement lequel empêche la formation de cette glace «à cause que les petites parties glacées de l'eau \& de ses [au fruit] sels ne pourroient pas se joindre ensemble »; le mouvement des particules de l'eau se communique à celles qui sont dans le fruit, ce qui rompt «le tissu » et le réduit « en une espéce de boüillie » lui faisant perdre son goût. De même, lorsque de la pluie tombe sur des corps après de fortes gelées, corps qui sont donc remplis de sels qui forment la glace, cette pluie se glace et donne du verglas car « les sels qui sont dans ces corps, \& qui y sont attachez au peu d'humide qu'il y a, le quittent facilement pour s'attacher à l'eau qui survient, dont ils forment une croute de glace »; si l'eau tombe trop abondamment, les sels ne la fixent plus, ils se mélangent avec l'eau et la glace se liquéfie ${ }^{82}$.

Lorsqu'il gèle, si on expose au vent un thermomètre qui était à l'air, «l'esprit de vin » descend considérablement, et si on couvre la boule de l'appareil de neige, «l'esprit de vin » remonte. Le vent transporte des sels et en fait passer à travers le verre plus que s'il ne soufflait pas; les particules d'eau dans «l'esprit de vin » s'en chargent, elles perdent du mouvement et occupent alors moins de volume, et «l'esprit de vin se condense ». Ensuite, sous l'action de la neige «l'esprit de vin»

\footnotetext{
${ }^{79}$ De par ces « assemblages » et ces « dilatations » il y aurait pour un même volume moins masse de glace que d'eau.

80 LA HiRE, «Explication des principaux effets de la Glace,», 1694, p. 98-99. La Hire précise qu' 'il se forme quelquefois plusieurs bulles par les differentes séparations qui se sont rencontrées dans l'eau avant qu'elle fust tout-àfait glacée, \& qui l'ont divisée comme en plusieurs vases qui contenoient de l'eau preste à se geler entierement ».

${ }^{81}$ Ibid., p. 200. Les p. 100 à 113 de ce mémoire sont notées par erreur 200 à 213.

82 Ibid., p. 201.
} 
remonte car les sels joints à ses particules d'eau « s'en détachent facilement pour se joindre à celle[s] de la nége, qui est plus propre à les retenir, en ayant une plus grande quantité ». C'est aussi pour cela que lorsque des parties d'un corps sont gelées on leur applique de la neige " qui retire à soy les particules des sels qui s'étoient insinuées dans les chairs ${ }^{83} »$. On protège les fruits du gel en les couvrant d'un peu de paille et d'un drap mouillé ; le drap empêche la gelée du fruit car les particules de sels rencontrent l'eau qu'il contient, s'y arrêtent, et ne passent pas outre à moins qu'il n'en survienne une trop grande quantité pour être retenue par l'eau ${ }^{84}$.

Les liqueurs peuvent geler l'été. Il suffit d'entourer le vase qui les contient de glace ou de neige mêlées avec beaucoup de sels ; sans ces sels il n'y a pas de gel, et le salpêtre fait plus d'effet que le sel commun, et le sel d'ammoniac (chlorure d'ammonium) davantage encore. Ainsi, ces trois sels contenant une grande quantité de sels servant à la congélation augmentent « la force de la nége en se mélant parmi ceux qui y sont retenus ; \& pénétrant ensemble le vase que contient l'eau, ils la font prendre facilement ». Sans eux, les particules de sels que la neige contient « auroient de la peine à s'en [l'eau de la neige] détacher ", il n'y en aurait pas en grande quantité, et le peu qui migrerait vers le liquide du vase ne le ferait pas prendre à cause du «tres-grand mouvement » de ses particules. Une rivière peut se glacer en été si les parties volatiles des sels dans les carrières de sels communs et de salpêtre emportées par l'air s'insinuent dans l'eau ${ }^{85}$. L'eau salée devrait geler plus facilement que l'eau douce car le sel commun contient d'autres sels aptes à fixer l'eau. Mais tel n'est pas le cas car «les autres sels [que ceux qui fixent l'eau] qui y sont [dans la mer] en grande quantité, empeschent les petites parties qui sont gelées de se joindre les unes aux autres en se mettant entre deux, ce qui n'arriveroit pas de méme s'il y avoit de l'eau, dont les particules se peuvent joindre à celles qui sont déjà gelées en s'y arrestant par differens endroits, \& former toutes ensemble un massif de glace ${ }^{86} »$.

Enfin, La Hire énonce une «observation » sur le froid qui "paroist tres-considerable » et qui « favorise beaucoup [son] hypothese » : des vents de même « force » dans une même saison peuvent être chauds ou froids; il y a donc dans les vents froids quelque chose qui fait une «impression differente » de celle des autres vents. Or, puisque les vents ne sont qu'une « agitation de l'air » ce sont « quelques parties » qu'il contient qui provoquent ces différences : «je dis que ces parties ne sont autre chose que les sels qui servent à fixer l'eau, qui penetrent la peau quand ils sont agitez, avec plus de violence que lorsqu'ils flottoient librement dans l'air ${ }^{87}{ }^{\prime}$.

\subsection{L’originalité d'un mécanisme et le statut des « hypotheses » en physique}

La Hire semble donc faire siennes les particules d'eau et de sels de Descartes. Par ailleurs, la référence à Lemery témoigne d'une connaissance de la chimie corpusculaire de la fin du $17^{\mathrm{e}}$ siècle dont ces sels peuvent être aussi issus. Enfin, à l'instar de Gassendi, il attribue la cause du froid et du gel à des corpuscules spécifiques. Malgré tout, son explication du gel et ses interprétations d'observations ne reproduisent pas à la lettre celles d'aînés et de contemporains. La physique de La Hire est corpusculaire, basée sur les formes et les propriétés spécifiques des corps - la flexibilité des particules d'eau rend possible qu'elles « entortillent» des sels « roides »-, sur les mouvements et sur les chocs de corpuscules - par exemple, lorsqu'il y a trop d'eau à proportion des sels, les particules d'eau qui choquent celles jointes aux sels empêchent le gel du liquide -, mais son « hypothese » et ses mécanismes lui sont propres.

Ainsi, il n'évoque pas le rôle joué par la matière subtile contrairement à Descartes, Rohault ou Régis. Ses propos semblent suggérer l'action d'un « air subtil », à l'instar de Mariotte mais, d'une

\footnotetext{
83 Ibid., p. 202-203.

${ }^{84}$ Ibid., p. 204.

${ }^{85}$ Ibid., p. 203-204.

${ }^{86}$ Ibid., p. 212.

${ }^{87}$ Ibid., p. 206.
} 
part, La Hire ne donne pas de détails ${ }^{88}$ et, d'autre part, nous avons remarqué ci-dessus qu'il se réfère à l'éther dans d'autres travaux : il ne se montre donc pas aussi critique que Mariotte à l'encontre de ce milieu ${ }^{89}$. L'« entortillement» de particules d'eau autour de sels se rencontre chez Descartes, Rohault et Régis. Selon Descartes les particules d'eau peuvent « se roller \& s'entortiller » autour des sels, et elles se meuvent alors plus vite que s'il n'y avait pas de sels : la matière subtile n'agit que pour les faire s'enrouler et les faire "passer » l'une sur l'autre « sans pour cela changer aucun de leurs plis »; a contrario, sans sels, puisque les corpuscules d'eau sont pliables une partie de la " force » de la matière subtile est employée à «les degager les unes des autres ». Ainsi, comme l'eau gèle "lorsque la matiere subtile, qui est entre ses parties, n'a pas la force de les agiter », l'eau salée ne gèle pas facilement. Concernant le gel de l'eau dans un vase placé dans un mélange de neige et de sel commun, la matière subtile qui est " plus grossiere, ou moins subtile », et qui par conséquent a " plus de force » dans l'eau du vase que dans la neige, "va prendre sa place à mesure que les parties de la neige se rollent autour du sel en se fondant : car elle trouve plus de facilité a se mouvoir dans les pores de l'eau salée qu'en ceux de l'eau douce »; elle contraint la matière " plus subtile » qui est dans la neige à prendre le chemin inverse et à migrer vers l'eau, mais « elle [cette matière dite plus subtile] n'a point assés de force pour y entretenir l'agitation de cette eau » qui devient alors glace ${ }^{90}$. Ce mécanisme d'« entortillement » est semblable chez Descartes et chez La Hire mais il répond à des fins différentes : pour l'un, il justifie le transfert de la matière subtile d'un lieu vers un autre, pour l'autre il explique directement la cessation du mouvement des particules d'eau et la solidification.

Descartes évoque le «plus de facilité » du mouvement de l'éther, tandis que La Hire mentionne celle des corpuscules frigorifiques qui migrent d'un membre humain ou d'un fruit gelés vers la neige ou l'eau où on les place. Gassendi fonde cette « facilité » sur la forme " pyramidale » des corpuscules frigorifiques: "la ressemblance, \& convenance, ou conformité reciproque » des «figures » et « consequemment [des] mouvemens » implique une migration de ces atomes. La Hire ne s'appuie pas sur cette dernière argumentation et, plus généralement, il ne fait pas siens les corpuscules de Gassendi et leur forme spécifique qui permet à ce dernier d'expliquer la formation de la glace ; au demeurant, La Hire ne semble pas adopter tous les principes gassendiens, et en particulier le vide ${ }^{91}$. Ainsi, concernant le gel de l'eau par l'action du sel commun ou du salpêtre, Gassendi précise qu'il ne s'agit pas d'une "privation de chaleur de l'eau ", mais d'« une veritable, positive, \& active qualité » et fait intervenir des " petis corps penetrant au travers du verre » ${ }^{92}$; interprétation que La Hire adopte sans pour autant recourir aux corpuscules et au mécanisme de son ainé. Une interprétation qui est a contrario critiquée par son collègue académicien Mariotte qui pour sa part estime qu'il n'existe « aucune cause positive du froid » et qui dénonce l'usage des « esprits nitreux » et des « esprits [...] frigorifiques » : les corps possèdent seulement différents degrés de « chaleur » jusqu’à la « privation » de celle-ci ${ }^{93}$.

\footnotetext{
${ }^{88} \mathrm{La}$ Hire écrit qu'on pourrait « supposer que tout le mouvement des particules de l'eau ne leur vient que ce celuy de l'air subtil, qui y est toujours mélé en grande quantité », mais il ne s'attache ici qu’à la manière dont «l'eau peut perdre son mouvement, pour rendre raison des principaux effets du froid, \& de la glace ». Ibid., p. 97.

89 MARiotTe, Essays de physique. Second Essay, 1679, p. 152-153 se montre critique à l'encontre des explications cartésiennes basées sur la matière subtile et sur l'existence même de cette dernière qu'il juge «nullement necessaire pour les effets naturels ».

${ }^{90}$ Descartes, Les météores, 1996, p. 251-253. Voir RoHault, Traité de physique, 1671, p. 164-165 et REGIS, Systême de philosophie, 1691, t. 2, p. 203-204. Rohault précise que la matière subtile qui est dans les pores de la neige doit être "plus subtile ou moins agitée » que celle dans les pores de l'eau car « autrement elle auroit la force de l'entretenir liquide »; il souligne aussi qu'il circule « autant » de matière subtile de l'eau vers la neige que de la neige vers l'eau.

${ }^{91}$ Voir ci-dessus à la partie sur l'éther chez La Hire.

92 Bernier, Abregé de la philosophie de Gassendi, 1678, t. II, p. 107 et p. 117.

${ }^{93}$ MARIOTTE, Essays de physique. Troisieme Essay, 1679, p. 20-22 sur les différents degrés de chaleur et p. 28 pour le rejet des « esprits ». Mariotte interprète le refroidissement de l'eau d'un vase placé dans un récipient contenant un mélange d'eau et de salpêtre par le fait que ce dernier est plus «condensé que l'eau » et qu'«il communique plus fortement sa froideur que l'eau ». Un mélange de neige et de sel commun placé en contact et en-dessous d'un plat contenant de l'eau l'a fait geler plus rapidement que s'il n'y avait pas de sel, car ce dernier fond en partie et le mélange d'eau salée « touche
} 
La Hire énonce ainsi sa propre « hypothese », mais que faut-il entendre par ce terme ? Fontenelle écrit que La Hire ne prenait son explication du froid que "pour un Sistême où un principe vraisemblable étant posé, tout le reste s'en déduisoit assés bien », que La Hire « se contentoit d'avoir bien raisonné, sans prétendre avoir bien deviné » et, plus généralement, «qu’il ne croyoit pas que dans les matieres de pure Physique le secret de la Nature soit aisé à attraper ${ }^{94}$ ». Le commentaire de La Hire sur sa propre explication paraît confirmer ces propos, mélangeant à la fois satisfaction et prudence, sinon doutes, sur la certitude qu'on peut avoir en physique :

pour ce qui regarde les effets de la glace \& du froid, il me semble que le systême que j'ay pris pour expliquer ce que j'ay pû remarquer de considerable sur ce sujet, satisfait si bien à tout, qu'on pourroit dire qu'il seroit vray si l’on pouvoit avoir quelque connoissance certaine dans la Physique ${ }^{95}$.

Il est ici question de la certitude d'un «systême », autrement dit du degré de « vrai-semblable » d'un "principe », pour reprendre les mots de Fontenelle. Que celui-ci satisfasse aux phénomènes à l'issue de déductions pourrait donc ne pas suffire à ce qu'il soit «vray ».

Dans un manuscrit de 1692 édité en Annexe de ce volume, La Hire écrit considérer le système des tourbillons de Descartes «comme une hypothese dont on peut se servir pour expliquer commodement plusieurs mouvemens qu'on observe dans la nature ». Il estime aussi que le «systeme» que Descartes propose dans son explication des marées, et qui est basé sur ces tourbillons, « semble le plus probable de tous ${ }^{96}$ ». Ces propos se placent dans le cadre d'une défense de l'explication des marées de la quatrième partie des Principes de la philosophie de Descartes. Pour Descartes, la « science certaine » de la deuxième partie de ce livre laisse place dans les deux suivantes qui concernent le «monde visible » et «la Terre » à « une hypothese [les tourbillons célestes] laquelle peut estre fort éloignée de la vérité », le philosophe ajoutant « mais encore que cela fust, je croiray avoir beaucoup fait, si toutes les choses qui en seront déduites, sont entierement conformes aux experiences ${ }^{97}$ ». Descartes illustre le sens du mot «hypothese» par la pratique des "Astronomes » : ils ont inventé «trois differentes hypotheses ou suppositions [celles de Ptolémée, de Copernic et de Tycho-Brahé] qu'ils ont seulement tasché de rendre propres à expliquer tous les phainomenes, sans s'arrester particulierement à examiner si elles estoient avec cela conformes à la verité $^{98}$ ». L'accord entre la déduction à partir des principes du mécanisme et les faits expérimentaux ou les observations ne garantit pas pour Descartes qu'il s'agisse réellement de la voie suivie par Dieu dans sa création de phénomènes ${ }^{99}$. S’Il ne nous trompe pas, la seule vérité réside dans la possibilité de la relation cause-effet proposée, d'où cette mention d'une « hypothese ${ }^{100}$ ». Pour des phénomènes particuliers dont les expériences manquent pour trouver les «vrayes causes » il faut se contenter d'explications à titre d'hypothèses ${ }^{101}$.

Pour Régis la physique, à cause des parties « insensibles » dans la nature dont on n’aperçoit «ni l'ordre ni l'arrangement », requiert une dimension « Pratique » consistant en l'observation des effets et une autre «Spéculative » pour découvrir les causes de ces effets, domaine d'où est exclu ce qui

le plat supérieur en beaucoup plus de parties que ne fait neige seule ». Ibid., p. 23-25. En somme, la masse - le salpêtre est plus « condensé »- et la surface de contact permettent d'interpréter ces phénomènes sans recourir à des corpuscules particuliers.

94 FONTENELLE, «Eloge de M. de La Hire », 1719, p. 89.

${ }^{95}$ LA HIRE, Mémoires de mathématique et de physique, 1694, Préface, non paginée.

${ }^{96}$ LA HiRE, «Défense du système de m. Descartes», 1692, p. 2.

${ }^{97}$ Descartes, Principes, 1996, Partie III, AT IX, art. 44, p. 123. Les "principes » énoncés dans la seconde partie des Principes - la matière est étendue, les phénomènes s'expliquent par la figure, la grandeur et le mouvement de la matière, le mouvement suit certaines lois - peuvent donner lieu à plusieurs explications d'un phénomène et les observations et l'expérience permettent de choisir entre les voies déductives. Ibid., art. 4 et 46, p. 104-105 et p. 124.

98 Ibid., art. 15, p. 108.

${ }^{99}$ Ibid., Partie IV, art. 204, p. 322-323.

100 Ibid., Partie III, art. 43 et 44, p. 123.

101 Ibid., art. 132, p. 185. 
est « démonstratif ${ }^{102}$ ». Pour Régis l'hypothèse parait alors consubstantielle au mécanisme et la cohérence de l'ensemble déductif, et donc du système physique, est un critère validant la réalité du raisonnement proposé. Ainsi, " pour rendre la Physique la plus parfaite qu'il est possible, il faut nécessairement joindre la partie speculative à la partie pratique », et la physique est "obligée de recevoir tout ce qui est probable, pourvû qu'il soit déduit d'un seul Système fondé sur les premieres vérités de la nature ", un "système » correspondant alors à "un amas de plusieurs hypothèses dépendantes les unes des autres, \& tellement liées avec les premieres vérités qu'elles en soient des suites \& des dépendances nécessaires ${ }^{103} »$. Descartes évoque la «certitude morale » en utilisant la métaphore du « chiffre » dont on sait l'avoir déchiffré lorsque l'interprétation d'ensemble donnée tient ${ }^{104}$. Autrement dit, l'hypothèse et son caractère probable devient cause moralement certaine en faisant système lequel résorbe une tension entre, d'un côté, une science certaine et, de l'autre, la physique hypothétique ${ }^{105}$.

La Hire écrit que son "systême » pour le froid « satisfait [...] bien à tout », autrement dit aux phénomènes, sans pour autant qu'on puisse le dire «vray», le savant se montrant manifestement sceptique sur la possibilité d'avoir des «certitudes » en physique. Il écrit aussi que les tourbillons expliquent « commodement plusieurs mouvemens qu'on observe dans la nature », ce qui signifie qu'à partir de cet unique principe il est possible d'interpréter divers phénomènes et de rendre compte d'observations et d'expériences, le tout formant un ensemble cohérent. Faut-il alors accorder à La Hire une conception de l'hypothèse, du système et de la «certitude morale » en cohérence avec Descartes ou Régis ? De fait, l'« hypothese » intervient dans des contextes où La Hire se réfère à Descartes, et notamment aux Principes de la philosophie, soit qu'il adopte la nature de ses corpuscules, soit qu'il prenne la défense d'une de ses explications tourbillonnaires, et donc de l'« hypothese » des tourbillons. Ceci suggère que La Hire fait siennes ces conceptions, tout en soulignant qu'il propose peu de mécanismes, qu'il n'est pas un auteur à systèmes, et que ses travaux n'exposent pas explicitement son épistémologie de la physique. A contrario, la question des fondements de la mécanique rationnelle parait être une préoccupation majeure où il est possible d'énoncer des certitudes.

\section{Mécanique rationnelle, mécanique appliquée}

\subsection{La statique : principes et démonstrations}

D’après la «Préface » de son Traité de mécanique de 1695, La Hire conçoit que la «mécanique » relève de la science des machines, mais il évoque aussi d'autres "parties de cette Science » et en particulier de «nouvelles découvertes» qui n'ont pas «de si grands usages dans la vie civile que celles des Anciens en avoient dans leurs temps", mais qui les «surpassent» étant "des « explications, \& même des démonstrations exactes des effets les plus admirables de la nature ${ }^{106}$. Le propos semble se rapporter à cette évolution du sens du mot "mécanique » évoquée dans l'introduction, qui englobe le domaine des machines mais aussi des lois de la nature plus générales ; de fait, outre le levier, les moufles etc., La Hire traite dans ce livre de lois du choc ou encore de cinématique galiléenne. Ce traité aborde ainsi :

\footnotetext{
102 REgIS, Systême de Philosophie, 1691, t. II, «Avertissement», p. 3-5. Cet exemple de Régis est tiré de Roux, «Le scepticisme et les hypothèses », 1998, p. 237.

103 REGIS, Systême de Philosophie, 1691, t. II, «Avertissement », p. 5. Ces " premieres vérités » sont celles du mécanisme cartésien et sont énoncées dans la Partie II des Principes de la philosophie. Il s'agit de l'identification de la matière à l'étendue et des lois du mouvement des corps.

104 Une métaphore très présente dans la seconde moitié du 17e siècle, voir RouX, « Le scepticisme et les hypothèses ", 1998, p. 227-228.

105 Sur ces questions, nous renvoyons à Roux, « Le scepticisme et les hypothèses ", 1998.

106 LA Hire, Traité de mecanique, 1695, « Préface » (non paginée).
} 
a) les cinq machines simples de la statique (le levier, le treuil, la poulie, le coin, le plan incliné avec la vis) et la description de « quelques machines composées » de celles-ci (p. $1-381)^{107}$

b) le choc et ses règles (p. 382-403);

c) les centres d'oscillation et de percussion (p. 404-407);

d) la chute libre (mouvements sur un plan incliné, une cycloïde, jet de projectiles) (p. 408448) et un instrument pour le jet des bombes (p. 449-455) ;

e) des questions liées à la courbe caténaire et à la charge des voussoirs (p. 455-470) ;

f) la résistance des solides (p. 471-492) ${ }^{108}$.

L'opus est donc essentiellement consacré à la statique et aux machines, son sous-titre stipulant qu'« on explique tout [dans ce livre] ce qui est nécessaire dans la pratique des Arts, \& les propriétés des corps pesants lesquelles ont un plus grand usage dans la Physique ». Les parties b), c), d), f) s'inscrivent dans la lignée des travaux de Galilée, Mariotte et Huygens sans apporter de réelles nouveautés ${ }^{109}$. La section e) est étudiée en détail par A. Becchi ${ }^{110}$; P. Radelet-De-Grave donne un bref aperçu du contenu de l'œuvre, et met en regard quelques propositions de la partie a) avec les travaux de Varignon ${ }^{111}$. Pour notre part, nous examinerons dans la partie a) ce qui nous semble être deux aspects essentiels de l'ouvrage, à savoir son axiomatique et l'articulation d'un savoir théorique avec « la pratique des Arts ». Nous montrerons l'originalité de La Hire et comment elle le singularise de travaux de certains de ses collègues académiciens.

La Hire énonce des «premiers éléments » de statique et estime que parmi tous les ouvrages de mécanique des «Anciens », seuls ceux d'Archimède donnent «les principes de cette Science [...] traités à fond $»^{112}$. Il considère le «levier [...] comme la proposition fondamentale de toute la Mécanique, puisque toutes les autres parties peuvent s'y réduire facilement ${ }^{113} »$; les machines "peuvent toutes se rapporter au levier ${ }^{114}$ ». Il dénonce ceux qui, parmi les "Modernes", ont «abandonné les principes dont Archiméde s'est servi », et qui ont fait « des suppositions toutes nouvelles dont ils se servent pour principes dans leurs démonstrations ». La Hire estime ces « suppositions » insuffisantes pour « une bonne démonstration » et juge que ces auteurs emploient «sans en avertir» des principes supplémentaires qui mériteraient démonstrations ou au moins explications. Ceci est alors contraire « aux démonstrations rigoureuses de la Geométrie » et conduit à deux conséquences : ceux qui sont habitués à ces dernières ne sont pas convaincus « de quelques propositions où la Physique se trouve mêlée », et d'autres préfèrent «faire une supposition de la principale proposition [la loi du levier], que de n'en donner qu'une démonstration douteuse ou fondée sur des principes qui n’ont pas une tres-grande évidence ${ }^{115} »$; nous verrons ci-dessous que La Hire critique aussi des "principes d'expériences». Il entend démontrer la loi du levier dans la première proposition de son livre à l'aide de considération de symétrie. Il écrit :

\footnotetext{
107 Ibid., p. 331.

${ }^{108}$ Ce découpage et ces répartitions sont les nôtres, et non ceux de La Hire.

109 GALILEI, Le Mecaniche, 1891 et GALILEI, Discorsi e dimostrazioni matematiche, 1898 pour la chute libre ; MARIOTTE, Traitté de la percussion, 1673 pour les chocs, les centres d'oscillation et de percussion et la résistance des matériaux, HUYGENS, Horologium oscillatorium, 1673 pour la chute libre sur la cycloïde.

110 BECCHI, «Idées manuscrites, théories imprimées », 2013.

111 RADELET-DE-GRAVE, «La mécanique », 2013. Nous évoquerons cette comparaison ci-dessous.

112 LA HiRE, Traité de mecanique, 1695, « Préface » (non paginée).

113 BECCHI, «Idées manuscrites, théories imprimées », 2013, p. 178-179 souligne la référence à l'Antiquité et le silence au sujet d'auteurs de la Renaissance qui réhabilitent Archimède, en particulier Guidobaldo del Monte. Del Monte réduit l'étude de l'équilibre des machines simples à celui du levier, lui-même réduit à l'équilibre de la balance qui est la première «Demande » qui apparait dans ARCHIMEDE, De l'équilibre des plans, 1807, p. 275. Voir PALMIERI, « Breaking the circle », 2008. Sur Del Monte, voir Bertoloni Meli, « Guidobaldo dal Monte », 1992.

${ }^{114}$ LA HIRE, Traité de mecanique, 1695, p. 6.

115 Ibid., "Préface », non paginée. Nous donnerons, ci-dessous, des aspects de la critique de La Hire en examinant sa démonstration de la loi du levier.
} 
j’ay tâché [...] de démontrer toutes les propositions à la maniére des anciens Geométres, sans me servir d'autre Axiome ou proposition fondamentale que de celle que tous ceux qui ont écrit de Mécanique ont supposée d'abord [la loi du levier] ; \& pour la rendre encore plus évidente je la démontre dans ma premiére proposition par une autre qui est plus universelle \& dont on ne fait aucun doute dans la Physique, qui est que dans l'effort des puissances toutes choses étant égales d'un côté \& d'autre, les efforts sont égaux. C'est-pourquoy je n'ay pas crû qu'il fust nécessaire de rapporter ce principe, puisque c'est un des plus simples de toute la Physique ${ }^{116}$.

Au sujet des sens des termes "puissances» et " efforts", La Hire évoque «l'effort des puissances », les «cinq puissances ou machines principales ${ }^{117}$ ", les « efforts que peuvent faire les forces mouvantes par le moyen des machines ${ }^{118}$ ». Il précise aussi que dans une machine " on peut toûjours considerer trois puissances »dont une seule résiste à «l'effort» des deux autres : la "puissance monvante », «le poids ou la puissance qui est meuë », "l'appui » ou " puissance qui soutient» appelée aussi «Hypomocblion ${ }^{119}$ »; par ailleurs, « il peut aussi y avoir équilibre entre deux puissances qui seront directement opposées ${ }^{120} »$. Quand La Hire écrit que «l'effort d'une puissance peut être augmenté à l'infini ${ }^{121}$ ", il s'agit de dire qu'une " puissance » étant donnée, il est possible qu'elle produise davantage d'effet suivant sa disposition sur une machine; la « Mécanique » permet de connaitre comment son « effort » peut être « augmenté » par le biais d'un dispositif. La « puissance » renvoie donc à une « force » ainsi qu'à la machine elle-même. L'« effort » peut simplement renvoyer à un poids - « la pesanteur est l'effort que fait un corps pour descendre ${ }^{122} »-$, mais il s'identifie aussi à l'effet des «puissances » sur une machine. Selon La Hire « on appelle moment d'un corps pesant l'effort avec lequel il peut agir sur un autre corps lorsqu'il est appliqué à la machine ${ }^{123} \%$. Ainsi, «l'effort d'une puissance dépend de la distance entre l'appui \& l'endroit où elle est appliquée sur le levier [...] l'effort augmentera à proportion que cette distance sera plus grande »; il n'y aura pas, dans ce cas, davantage de charge sur l'appui, lequel n'est pas chargé « de l'effort des puissances ou poids [variable en fonction des distances à l'appui], ce qui est la puissance ou la pesanteur relative », mais seulement de leur « puissance ou pesanteur absoluë ${ }^{124} »$. Ainsi, pour reprendre les formules du savant, «l'effort des puissances » renvoie à cette notion de " puissance relative » laquelle s'identifie manifestement à un moment statique. Aussi, lorsque La Hire énonce la proposition " universelle » stipulant que « dans l'effort des puissances toutes choses étant égales d'un côté \& d'autre, les efforts sont égaux » il entend manifestement une égalité de moments. Par ailleurs, dans son "Traité des épicycloïdes », nous verrons qu'il recourt au principe statique de Descartes : dans ce cas, l'« effort» correspond au déplacement d'une «puissance » le long de la verticale.

Comment s'articulent la «Proposition [...] universelle» et l'«Axiome ou proposition fondamentale », et quelles méthodes La Hire met-il en œuvre pour établir la loi du levier ?

La Proposition I, ou "proposition fondamentale», stipule qu'avec les poids $D$ et $E$ égaux (Fig. 1), dont les droites d'action sont parallèles entre elles et sont perpendiculaires au levier, et avec une "puissance » $H$, selon la terminologie de La Hire, parallèle aux deux autres et égale à leur somme, alors $D, E, H$ s'équilibrent. Dans sa "démonstration», La Hire écrit que $D$ et $E$ étant égaux, ayant des directions parallèles, et étant " également éloignés de leur point d'appui $B$ », « il est certain que l'un des poids ne peut surmonter l'autre, puisque toutes choses sont égales des deux côtés ; c'est pourquoy ces deux poids demeureront en équilibre entr'eux ». La Hire établit ensuite l'intensité de «la puissance $H$ qui soutient le levier en $B$ » : elle « pousse le point $B$ avec un effort double de chacun des poids ${ }^{125}$ ». La symétrie de la configuration fonde donc l'équilibre ; La Hire

\footnotetext{
116 Ibid.

117 Ibid., p. 5.

118 Ibid., p. 1.

119 Ibid., p. 6.

${ }^{120}$ Ibid., p. 6-7.

${ }^{121}$ Ibid., p. 1-2.

122 Ibid., p. 7.

${ }^{123}$ Ibid., p. 9.

124 Ibid., p. 42-43.

125 Ibid., p. 14-16.
} 
s'appuie finalement sur une proposition « universelle » identifiable à la première « demande » de De l'équilibre des plans d'Archimède ${ }^{126}$.

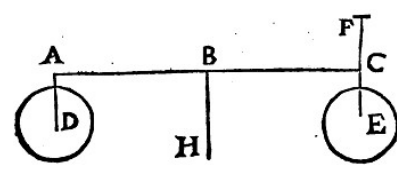

Fig. 1: LA HiRE, Traité de mecanique, 1695, p. 15

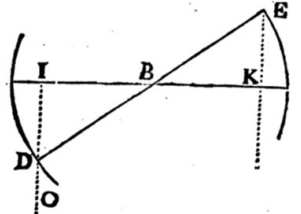

Fig. 2 : LA HiRE, Traité de mecanique, 1695, p. 18

La Proposition II établit la loi du levier pour des «puissances » $E$ et $D$ (Fig. 2) parallèles entre elles mais non orthogonales au levier. La Hire trace l'horizontale $I B K$ et les directions normales $I D$ et $E K$ des forces : par la «troisième supposition» qui énonce que «les corps pesans pesent egalement dans tous les points de leur ligne », et par la géométrie du problème, il se ramène au levier de la Proposition I ${ }^{127}$.

Les Propositions I et II servent à établir la Proposition III qui énonce que si deux poids « en raison de nombre à nombre» sont placés de part et d'autre du point d'appui d'un levier en proportion inverse des longueurs des bras, alors ces derniers s'équilibrent et l'appui est chargé de ces deux poids. Le ressort de la démonstration repose sur deux leviers identiques $A B C$ et $D E F$ (Fig. 3). Le premier, de point d'appui $B$, chargé de deux poids $A$ et $C$ de 2 livres, avec $A B=$ $B C$, et le deuxième, de point d'appui $E$, avec $D$ et $F$ de 1 livre et $D E=E F(=A B=B C)$. La Hire place $E$ du deuxième levier en $A-D E F$ devient def -, ce qui donne la configuration de la Figure II pour $d$ et $f$, puis il place $d e f$ comme représenté dans la Fig. 4 ; dans les deux cas, l'équilibre demeure. Dans la Fig. 4, les deux leviers n'en font qu'un d'appui $B, 1$ livre en $d$ équilibrant 2 livres en $C$. En fixant $f$ en $B, d$ fait un « effort» de 1 livre pour élever $f$, tandis que $B$ agit d'un « effort» de 4 livres dans le levier $A B C$ : ainsi l'« effort» de 1 livre en $d$ «soulage » le poids de 4 livres en $B$ " en élevant ce mesme point $B$ qui est joint au point $f$, avec un effort de 1 livre ». Par conséquent, $d$ de 1 livre et $C$ de 2 livres qui s'équilibrent sont dans le rapport de $B C$ à $B d$, et la « puissance » en $B$ qui « soutient $~ C$ et $d$ est de 3 livres ${ }^{128}$.

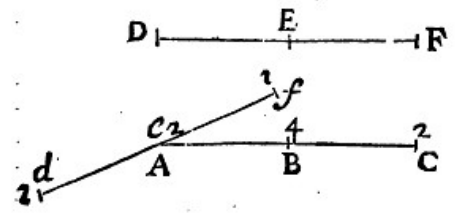

Fig. 3 : LA HIRE, Traité de mecanique, 1695, p. 20

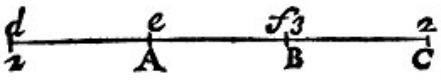

Fig. 4 : LA HIRE, Traité de mecanique, 1695, p. 20

La Proposition IV s'attache à l'équilibre dans «quelque rapport que les poids puissent avoir entr'eux » en s'appuyant notamment sur la Proposition III, et donc la Proposition I ${ }^{129}$. Elle débute par un Lemme qui stipule qu'un poids plus éloigné d'un point d'appui qu'un autre poids égal fait plus d'« effort » que ce dernier. La démonstration du Lemme s'appuie sur le même ressort que la Proposition III en recourant à deux leviers. La Hire considère le levier $D H E$ d'appui $H$ avec les poids $A$ et $B$ (Fig. 5). Il démontre par l'absurde que $A$ fait plus d'effort en $C$ qu'en $D$. Il suppose que $A$ en $D$ fait autant ou plus d'effort sur $B$ que s'il était en $C$. Il place en $D$ l'appui $D$ du levier $I D F$, avec $I D=C D$ et $D F=A H$, chargé de $G$ et de $L$, de telle sorte que ce levier fasse «le même effet » que $A$. La Hire remarque que le poids $G$ pèse moins que $A$, puis il place $F$ en $H$ et $I$ en $C$.

\footnotetext{
126 Pour cette «demande », voir ARCHIMEDE, De l'équilibre des plans, 1807, p. 275 : «Des graves égaux suspendus à des longueurs égales sont en équilibre ».

127 LA HiRe, Traité de mecanique, 1695, p. 17-19 et p. 11. La première «supposition » considère les droites d'action des poids parallèles entre elles « quoy qu'on croye qu'elles tendent vers le centre de la terre ».

128 Ibid., p. 19-30.

129 Ibid., p. 33-38.
} 
Si $A$ en $D$ faisait équilibre avec $B$ en $E$, il en serait de même avec $G$ appliqué en $C$. Par hypothèse, $A$ en $C$ « ne fait qu'autant ou même moins » que $A$ en $D$. Mais $G$ placé en $C$ fait « autant ou plus d'effort » que $A$ en $C$ car il en fait autant que $A$ en $D$ dans le cas où $A$ équilibre $B$ : ceci est absurde car $G$ moins pesant que $A$ placés tous les deux en $C$ ne saurait faire plus d'effort ou un effort égal à $A$. Ainsi $A$ en $D$ ne peut pas faire un effort égal ou plus grand que $A$ en $C$. La Hire ajoute en commentaire que "cette proposition a esté mise en axiome par quelques Geométres » et que "d'autres l'ont supposée sans en rien dire »; il précise que « ce n'est pas une chose si claire qu'elle puisse estre receuë sans difficulté, \& l'on ne pourroit tout au plus la supposer que comme un principe d'experience ${ }^{130} \%$. Archimède et Huygens prennent cet énoncé comme une demande ${ }^{131}$; l'enjeu est donc bien d'établir une axiomatique «claire » et si possible indépendante de « principes d'experiences ».

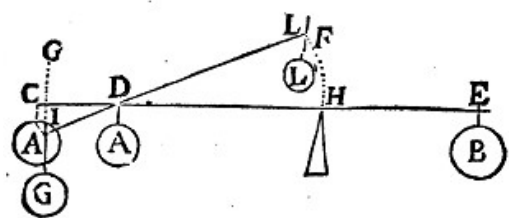

Fig. 5 : LA HIRE, Traité de mecanique, 1695, p. 20

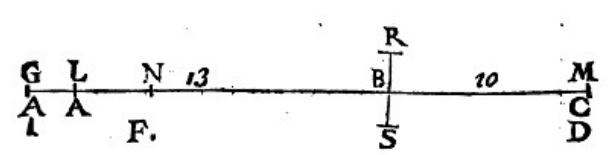

Fig. 6 : LA HIRE, Traité de mecanique, 1695, p. 34

La Proposition IV établit que si le levier $L M$ (Fig. 6) d'appui B est tel que $A . B L=C . B M$ alors il y a équilibre. S'il n'y a pas équilibre, $A$ l'« emporte » $\operatorname{sur} C$. Il faut donc ajouter $D$ à $C$ de telle sorte que l'équilibre ait lieu. La Hire suppose que $A$ soit divisé en 10 poids égaux à $F$ de telle sorte que $13 F>C$ et $13 F<C+D$ et divise $B M$ en 10 parties égales entre elles. $B L$ ne " contient » pas 13 de ces parties $\operatorname{car} \frac{13}{10} A(=13 F)>C$, or $\frac{A}{C}=\frac{B M}{B L}$, donc $\frac{13}{10} B M>B L$. Ces 13 parties de $\frac{B M}{10}$ donnent la distance $B G$. D'après la Proposition III, $13 F$ en $M$ équilibrent $A=10 F$ en $G$. Mais par construction $13 F<C+D$, donc $A$ en $G$ ferait équilibre avec un poids moindre que $C+D$ et il faut donc ajouter un poids $I$ à $A$ pour avoir l'équilibre avec $C+D$. Ainsi, le seul poids $A$ qui en $L$ équilibrait par hypothèse $C+D$ en $M$ ne peut équilibrer $C+D$ qu'avec le secours d'un poids supplémentaire $I$ lorsqu'il est plus éloigné que $B L$. Or, d'après le Lemme précédent, $A$ fait un effort moindre en $L$ que dans cette deuxième configuration. Ainsi, $A$ et $C+D$ ne s'équilibrent pas, ou encore $A$ n'« emporte » pas $C$, suivant le mot de La Hire, lorsque $A . B L=C . B M$, mais les deux poids s'équilibrent.

Ainsi La Hire s'appuie sur un principe "universel" semblable à la première "demande" de De l'équilibre des plans d'Archimède. La loi du levier est établie pour des grandeurs commensurables puis incommensurables chez les deux auteurs, et en particulier par des procédés de réduction par l'absurde pour des poids incommensurables chez l'un comme chez l'autre. La Hire remplace aussi des poids par des leviers équilibrés, ce qui pourrait être rapproché de la technique d'Archimède à l'œuvre dans la Proposition VI lorsqu'il décompose une charge en plusieurs autres de part et d'autre de son centre de gravité ${ }^{132}$. La Hire ne suit pas à la lettre les démonstrations d'Archimède mais il nourrit le même projet de fonder géométriquement la statique, au point d'aller plus loin que le maitre en cherchant à la rendre indépendante de certains "principes d'expériences" qu'il juge pour sa part démontrables.

\footnotetext{
130 Ibid., p. 30-32.

131 ARCHIMEDE, De l'équilibre des plans, 1807, p. 275. HuyGENS, « Démonstration de l'équilibre de la balance », 1693, p. 313-316. Ce mémoire fait manifestement partie des manuscrits envoyés par Huygens à La Hire pour la publication de ce volume académique. Voir HuYGENS, Mécanique théorique et physique de 1666 à 1695, 1937, p. 17-18, et les lettres de La Hire à Huygens du 8 septembre 1686, p. 91-92, de Huygens à La Hire, du 26 septembre 1686, p. 95-97 et de Huygens à D’Alencé du 20 juin 1687, p. 167, dans HuYGENS, Correspondance 1685-1690, 1901.

132 ARCHIMEDE, De l'équilibre des plans, Proposition VI et VII, p. 280-281.
} 
La Hire n'adopte pas les principes de statique de Descartes, Galilée, Wallis ou encore Mariotte ${ }^{133}$. Il ne suit pas davantage l'axiomatique développée par son confère académicien Pierre Varignon qui base l'étude des machines simples sur la composition de forces : l'équilibre du levier requiert que la réaction du point d'appui soit dans la direction de la résultante des forces appliquées aux bras et d'intensité égale à cette résultante ${ }^{134}$; composition et équilibre de la résultante figurent bien chez La Hire mais elles résultent d'applications de la loi du levier qui a donc axiomatiquement la prévalence ${ }^{135}$. La Hire dénonce les «Modernes » ayant «abandonné les principes » d'Archimède vis-à-vis duquel Varignon prend justement explicitement ses distances. Ainsi, Varignon estime non « raisonnable » l'étude de l'équilibre des machines simples à l'aide du levier car il juge qu'elles sont toutes aussi « simples » les unes que les autres : les machines ne doivent pas avoir de « dépendance » à l'égard du levier et « ceux qui les y rapportoient, n'y étoient forcez que parce que leurs principes n'avoient pas assez d'étenduë ». De manière générale, Varignon critique les auteurs qui « sont plus attachés à prouver la nécessité de l'équilibre, qu'à prouver la maniére dont il se fait », et cherche $a$ contrario cette «maniére » en s'appuyant sur le concept de force ${ }^{136}$. Ce type de critique pourrait être notamment adressé à l'encontre d'un discours de nature synthétique et des procédures de réduction par l'absurde qui énoncent qu'une égalité de moments statiques correspond à un équilibre, et que supposer un déséquilibre dans ce cas implique contradiction; avec sa méthode, Varignon montre que l'équilibre implique une égalité de moments et il établit ainsi l'origine de cette relation ${ }^{137}$. En somme, les approches de La Hire et Varignon diffèrent tant au regard des principes que des méthodes.

La Hire n'utilise pas des principes dans lesquels interviennent le temps ou les vitesses, un choix qu'il faut peut-être lier à sa dénonciation de la "Physique" ou de principes "d'expériences" dans ce qui ne devrait reposer que sur des "démonstrations rigoureuses de la Géométrie », en reprenant ses expressions. Parmi les "modernes", Galilée énonce un principe de statique renfermant le temps et les vitesses ${ }^{138}$, et des successeurs de Descartes remplacent la dimension spatiale de son principe par une vitesse ${ }^{139}$. Mais Descartes récuse l'utilisation de cette dernière car il estime qu'il faudrait alors prendre en compte le milieu et la résistance qu'il oppose, et expliquer aussi ce qu'est « la pesanteur \& ensemble tout le système du monde » puisque la vitesse est liée à la pesanteur et à ses variations ${ }^{140}$. Réduisant la statique à un principe en insistant sur son évidence et son caractère apriorique, Descartes axiomatise cette science $^{141}$. Bien que se plaçant dans une tradition archimédienne, La Hire partage sans doute ce projet avec Descartes : il axiomatise la statique, il la fonde sur des principes aprioriques "évidents" et "clairs" pour reprendre sa terminologie et il vise

\footnotetext{
${ }^{133}$ Sur ces principes, voir SCHMIT, « Rapports entre équilibre et dynamique », 2014. Au regard des grandeurs physiques de ces principes, ils sont assimilables à ceux des travaux ou des vitesses virtuelles.

134 VARIGNON, Projet d'une nouvelle mechanique, 1687.

135 Pour une comparaison avec Varignon, voir RADELET-DE-GRAVE, « La mécanique », 2013.

136 Pour ces critiques, voir VARIGNON, Projet d'une nouvelle mechanique, 1687, « Préface » (non paginée). Sur cette genèse de l'équilibre et la force voir SCHMIT, « Rapports entre équilibre et dynamique », 2014, p. 531-533.

137 Nous verrons ci-dessous des critiques, notamment de Leibniz, à l'encontre La Hire pour son usage du style "synthétique" des "anciens". Soulignons que Varignon critique aussi les principes de Descartes et de Wallis qui ne montreraient pas comment « se fait » l'équilibre.

${ }^{138}$ GALILEI, Le Mecaniche, 1891, p. 164 et Dialogo sopra i due massimi sistemi del mondo, 1897, p. 241. Le Dialogo énonce que « la velocità del mobile meno grave compensa la gravità del mobile più grave e meno veloce ", et Galilée recourt à un principe de compensation entre un poids et une vitesse pour justifier l'équilibre d'un levier, voir sur cet aspect notamment GALluZZI, Momento. Studi galileiani, 1979, p. 207-212, Clavelin, La philosophie naturelle de Galilée, 1968, p. 162-167. LAGRANGE, Méchanique analitique, 1788, p. 8 attribue à la découverte du principe des vitesses virtuelles à Galilée.

${ }^{139}$ Le principe de Descartes repose sur l'idée que « la mesme force » pour élever un poids sur une certaine hauteur que permet d'élever un poids double sur une hauteur deux fois moindre. Voir la lettre de Descartes à Huygens du 5 octobre 1637, CEuvres de Descartes, vol. I, p. 435-436. Parmi ces successeurs, voir en particulier POISSON, Traité de la méchanique, 1668 , p. 22 , et p. $44-45$.

${ }^{140}$ Lettre de Descartes à Mersenne du 12 septembre 1638, Euvres de Descartes, vol. II, p. 355. Sur ces questions, voir les analyses de Roux, «Cartesian Mechanics », 2004.

${ }^{141}$ Sur ces aspects voir RoUX, « Cartesian Mechanics », 2004, p. 42-52.
} 
à lui conférer la même certitude que la géométrie ; par ailleurs, outre la loi du levier, La Hire recourt aussi dans son «Traité des Epicycloïdes » au principe de Descartes.

\subsection{Mécanique appliquée}

\subsubsection{Epicyclö̈des et roues dentées}

La Hire écrit que la dernière partie de son Traité de mécanique contient « ce qu'on a trouvé de plus curieux dans la Physique par rapport à la Mécanique », et il évoque alors notamment ses travaux sur « la forme des bras des moulins qui font jouer des pistons \& qui sont d'un tres-grand-usage » et « la construction d'une rouë qui sert à élever de l'eau où le frotement n'est pas sensible, [...] comme je l'ay fait éxécuter dans le Château de Beaulieu proche de Paris, \& dont la premiére invention étoit deuë à $\mathrm{M}$. Desargues ${ }^{142} »$. Il ne donne dans ce livre qu'un résumé du "Traité des Epicycloïdes » qui contient le détail des travaux théoriques qui conduisent à la «construction » de ces « bras » des moulins et de cette « rouë». C'est dans ce dernier traité qu'il rapporte que Desargues n'a pas « expliqué » le profil des dents de la roue, et qu'il en aurait seulement « déterminé la figure méchaniquement ». La Hire précise aussi que le propriétaire du château se serait adressé à Auzout et Mariotte "pour avoir le trait des dents ou ondes de cette rouë» et que ces derniers «me le renvoyèrent» car il les aurait informés environ vingt ans avant la publication du «Traité des Epicycloïdes » avoir « déterminé d'une manière tres-simple, que les dents des rouës devoient avoir la figure d'une [...] Epicycloïde ${ }^{143} »$. À quel objectif répond le profil de ces dents et comment La Hire parvient-il à leur attribuer cette « figure »?

La Hire écrit que les traités des « Mechaniques » ont plus abordé l'aspect « geometrique » de cette science que «l'execution» et, en particulier, «la figure des dents des rouës» a été «abandonnée » à la « pratique » et à «l'ouvrier»; il se propose d'« examiner avec un tres-grand soin » cette figure. Il entend remédier à l'à-peu-près par le recours à la géométrie et aux propositions premières de la mécanique. Il s'agirait de pallier «les frottemens » dus à des engrenages qui n'ont pas la figure convenable, frottements qu'on cherche habituellement à surmonter en donnant plus de «force » que nécessaire au fonctionnement normal de la machine. L'enjeu est donc l'efficience de la machine et la réduction de la dépense nécessaire - et en partie perdue - à son bon fonctionnement ${ }^{144}$. Mais, plus qu'à éliminer les frottements, ce que recherche véritablement La Hire est « l'application du mouvement égal aux machines », de « rendre le mouvement égal », ou encore qu'une "puissance $[. .$.$] agisse toûjours également \& non par sauts, comme il arrive à la pluspart$ des machines ${ }^{145}$ ». La nouvelle forme déterminée pour cet effet n'annule pas les frottements des dents entre elles. Au sujet de ceux-ci, et avec de telles dents, La Hire précise que les dents de deux roues doivent se «rencontr[er] en s'écartant» et non en "rentr[ant] l'une sur l'autre»: dans le premier cas, elles « ne se frottent qu'en échappant, ce qui n’apporte pas un empeschement sensible dans le mouvement », dans le deuxième, « l'empeschement au mouvement est fort considerable ${ }^{146} »$. Ces propos montrent que dans l'esprit du savant l'élimination de frottements repose sur la manière dont les pièces s'encastrent et agissent et n'est pas liée à leur forme épicycloïdale. Lorsque l'académicien Camus reprend cette question en vue d'une application à l'horlogerie, il précise bien

142 LA HiRE, Traité de mécanique, 1695, « Préface » (non paginée).

${ }^{143}$ LA HIRE, «Traité des Epicyloïdes », 1694, «Preface » (non paginée). Séris évoque cette histoire ainsi que la question de l'invention et de l'application des épicycloïdes aux dents des roues, en rapportant que, selon Leibniz, Roemer fit en premier cette application. Mais l'historien rapporte aussi qu'il n'est pas certain que Roemer et La Hire aient eu le même projet, celui-là visant à donner une forme de roues produisant un roulement sans frottements, quand celui-ci entendait que la forme assure une transmission continue du mouvement comme nous le verrons ci-dessous. SERIS, Machine et communication, 1987, p. 178-192. Sur la priorité de Roemer selon Leibniz, voir sa lettre à Jean I Bernoulli du 18 (28) janvier 1698 dans LEIBNIZ, Sämtliche Schriften und Briefe, 2011, p. 729-730.

${ }^{144}$ LA HiRE, « Traité des Epicyloïdes », 1694, «Preface » (non paginée).

145 Ibid., p. 55, p. 73 , et p. 69.

146 Ibid., p. 67, p. 70, et p. 78. 
que l'enjeu est de donner pour forme aux dents des roues et des pignons d'une horloge « celle qui tend à la faire marcher avec une force \& une vitesse uniforme, \& qui fait que les piéces font toûjours les unes sur les autres des efforts égaux ${ }^{147}$ ». Une « égalité de force » doublement nécessaire pour, à la fois, faire fonctionner l'horloge uniformément, et ce avec «la moindre puissance motrice qu'il est possible ». En effet, si le fonctionnement n'est pas « uniforme », autrement dit si les pièces agissent les unes sur les autres avec des « forces » variables, on a besoin de la " puissance motrice » nécessaire dans la situation la plus désavantageuse de ces pièces pour faire fonctionner le mécanisme ${ }^{148}$. Camus évoque ensuite le travail de La Hire qui consiste à étudier les formes à donner "aux dents des rouës pour qu'elles meuvent un pignon avec une vitesse toûjours égale à celle qu'elles ont-elles-mêmes ${ }^{149}$.

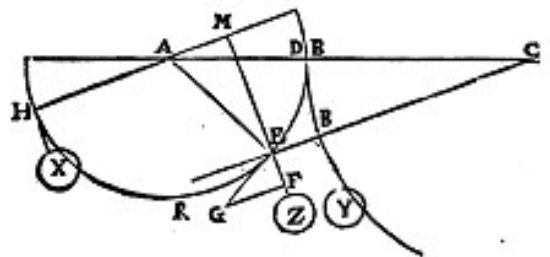

Fig. 7 : LA HiRe, Traité des Epicyloïdes, 1694, p. 52

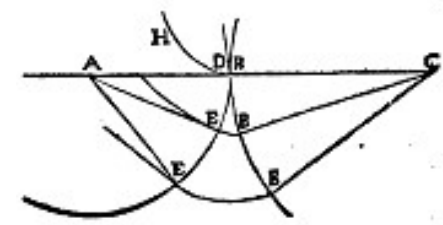

Fig. 8 : LA HiRE, Traité des Epicylö̈des, 1694, p. 53

Comment La Hire détermine le profil des dents ? La transmission d'un effort égal au cours du temps des engrenages sur les pignons implique qu'il faut un même contrepoids appliqué au pignon pour équilibrer l'action de l'engrenage; telle est la méthode mise en œuvre en recourant essentiellement au principe du levier.

Dans un premier temps, il suppose dans un même plan une ligne « déterminée » $A D$ (Fig. 7) qui peut tourner autour de $A$ de $D$ vers $E$, et une autre « indéterminée » $C B$ tournant autour de $C$, avec $B$ qui agit sur $D$ par son mouvement autour de $C$ vers le bas. Dans la position $A D C$, s'il y a équilibre entre ces lignes, les mêmes "puissances » $X$ agissent en $D$ et $B$. Mais si les lignes changent de direction, $A D$ devenant $A E$ et $C B$ prenant la direction $C B E$, La Hire montre que l'équilibre requiert en $B$ une «puissance » $Y>X$. Il considère deux leviers, $C E$ chargé de $Z$ en $E$ dont la direction d'action est $E F$, et $H A E$ réductible au levier droit $H A M$ dans lequel $X$ en $H$ équilibre une même «puissance » $X$ en $E$ de direction $E G$. L'équilibre entre $X$ et $Z$ conduit à $\frac{X}{Z}=\frac{A M}{A H(=A)}$. Si au lieu de $Z$ en $E$ on applique un poids $Y$ en $B$ qui fasse le même effet, on aura $\frac{Y}{Z}=\frac{C E}{C B}$. La combinaison des deux proportions donne $\frac{X}{Y}=\frac{A M}{A E} \cdot \frac{C B}{C E}$. Ainsi, lorsque $E$ est en $D$, on trouve $X=Y$ pour l'équilibre et, dans l'autre configuration $C B E$, puisque $A M<A E$ et $C B<C E$, alors $X<Y$. Ainsi, la

\footnotetext{
${ }^{147}$ Camus, « Des Dents des Roues », 1735, p. 117.

${ }^{148}$ L'horloger LE ROY dans «Dent», Encyclopédie, 1754, t. IV, p. 840b écrit que « la figure des dents des roues est une chose essentielle, \& à laquelle on doit faire beaucoup d'attention dans l'exécution des machines. On peut avoir parfaitement calculé le rapport des roues aux pignons, \& en conséquence l'effet que doit faire telle ou telle puissance dans une machine ; mais si la figure des dents des roues \& des ailes des pignons sur lesquelles elles agissent, n'est pas telle qu'il en résulte un mouvement uniforme de ces pignons, c'est-à-dire que l'effort que font les roues pour les faire tourner, ne soit pas constamment le même, un pareil calcul n'apprendra rien du véritable effet de la machine : car l'effort des roues étant tantôt plus grand, tantôt plus petit, on ne pourra tabler que sur l'effet de la machine dans le cas le plus desavantageux ; effet qui sera souvent très-difficile à connoître ». Voir aussi l'évocation des travaux de La Hire sur ce sujet dans l'article « Pignon », Encyclopédie, 1765, t. XII, p. 615b-616b.

149 CAmus, «Des Dents des Roues », 1735, p. 117-118. Camus remarque La Hire détermine la forme des dents d'une roue qui agit sur les fuseaux cylindriques d'une lanterne. En supposant des profils droits pour les dents des pignons, La Hire se sert d'une épicycloïde pour, par construction, obtenir les profils des dents des engrenages qui agissent sur ces pignons : Camus estime la méthode « sujette à plusieurs inconvénients » et, en particulier, La Hire ne donne pas la nature de la courbe des profils des engrenages mais l'obtient seulement « graphiquement». Voir aussi le reproche de LE RoY, «Dent », Encyclopédie, 1754, t. IV, p. 841a. Pour la méthode de La Hire, voir "Traité des Epicyloïdes », 1694, p. 63-65.
} 
«puissance » en $\mathrm{B}$ n'agit pas « également» en $D$ et en $E$ : les positions différentes requièrent des forces d'intensités différentes pour produire les mêmes effets ${ }^{150}$.

Ceci posé, La Hire décrit une épicycloïde $B H$ (Fig. 8), en quelque endroit que se trouve $B$ dans la rotation de $C B$ autour de $C$, avec la circonférence $B B$ du cercle centre $C$ et de rayon $C B$ et $D E R$ pour cercle générateur celui de centre $A$ et de rayon $A D$. $D$ prend les positions successives $E, E$, sous l'action de $C B, E$ étant un point de l'épicycloïde tirée de $B$. La Hire montre alors que «la puissance $X$ qui est appliquée en $E$ à l'extrémité de $A E$, comme elle estoit dans la Proposition précédente, sera en équilibre avec la mesme puissance $X$, qui est appliquée en $B$ à l'extrémité $B$ de la ligne $C B, \&$ qui agit sur le point $E$ par le moyen de la courbe de l'Epicyclö̈de $B E$ ». Il remarque que de par la formation de l'épicycloïde, en quelque position que se trouve $C B$, les arcs $B B$ et $D E$ seront égaux et, par conséquent, «la puissance appliquée en $B$, ne pourra se mouvoir par un arc $B B$ sans faire mouvoir l'autre puissance appliquée en $E$ à l'extrémité du levier $A E$ par un arc $E E$ égal en longueur à l'arc $B B$; d'où il suit que ces puissances estant égales, elles demeureront en équilibre ${ }^{151} »$. Manifestement, La Hire recourt ici à un autre principe de statique que celui de l'équilibre du levier et qui peut être rapproché de celui de Descartes. On retrouve ce principe dans une variante à cette démonstration basée sur le fait que $X$ (Fig. 9) qui agit le long de $E G$ sur le bras $A E$ équilibre $V$ de direction $E F$ qui agit sur $C E:$ «il faut que $X$ soit à $V$, comme $E F$ à $E G$, c'est à dire en raison reciproque des chemins parcourus ». Par construction, avec $N$ sur le diamètre $D A N$ du cercle générateur, $E N$ est la tangente en $E$ de l'épicycloïde ${ }^{152}$, et ainsi quand $E$ de l'extrémité de $C E$ parcourt « un espace $E F$ indéfiniment petit », $E$ à l'extrémité du levier $A E$ parcourt $E G$ avec «la petite portion $F G$ de la touchante $E N$ » qui «peut estre considerée comme la courbe elle mesme ». La Hire prend donc en compte ici des espaces « indéfiniment petits » franchis par l'action de forces et dans leur direction ${ }^{153}$.

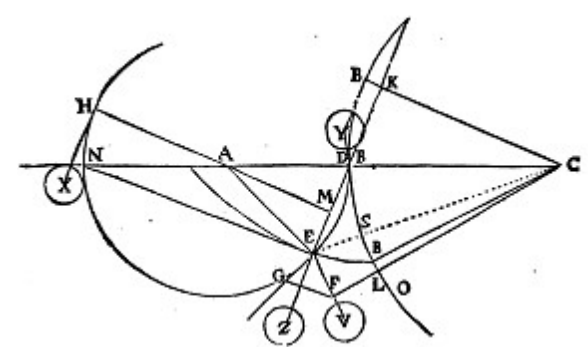

Fig. 9 : LA HiRE, Traité des Epicylö̈des, 1694, p. 54

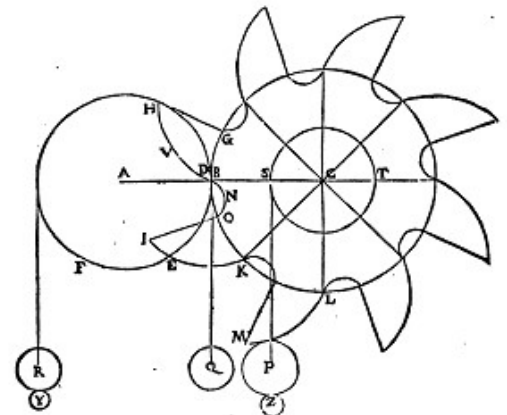

Fig. 10 : LA HiRE, Traité des Epicylö̈des, 1694, p. 56

La Hire propose aussi une autre démonstration qui, cette fois-ci, repose sur les mêmes ressorts que la première proposition ci-dessus. Ainsi, l'équilibre sur le levier $H A E$ (Fig. 9) requiert en $H$ et $E$ une puissance $X$ d'une même intensité avec les directions $H X$ et $E G$ normales à $A H$ et à $A E$. Selon la direction $E D$ (avec $D$ et $B$ confondus) normale à la tangente en $E$ de l'épicyclö̈de, $Z$ qui agit dans une direction normale à l'épicycloïde en $E$ équilibre $X$ sur le levier coudé $H A E$, réductible au levier droit $H A M$, si $\frac{X}{Z}=\frac{A M}{A H}$. Avec $C B$ parallèle à la tangente $E N$, et donc la « puissance » $Y$ en $B$ agissant dans la même direction que $Z$, cette dernière appliquée en $K$ et $Y$ en B s'équilibrent

${ }^{150}$ LA HiRE, « Traité des Epicyloïdes », 1694, p. 51-53.

151 Ibid., p. 53.

152 Ceci est établi dans la section mathématique du traité qui concerne les courbes épicycloïdes, ibid., p. 13-16.

153 Ibid., p. 54. Pour son principe, Descartes évoque la prise en compte du « commencement du mouvement » dans la direction des forces, autrement dit un petit espace sans doute à rapprocher de l'espace «indéfiniment petit» de La Hire. Voir sur ce point Roux, « Cartesians Mechanics », 2004. 
lorsque $\frac{Z}{Y}=\frac{C B}{C K}$. La combinaison des deux proportions donne $\frac{X}{Y}=\frac{A M}{A E} \cdot \frac{C B}{C K}$ avec $A H=A E$, et puisque les triangles $A M E$ (ou $A M D$ ) et $C K B$ sont semblables, il vient $X=Y^{154}$.

La Hire applique alors une même forme épicycloïdale $B V H$ (Fig. 10) aux dents d'une roue $B O L$ de centre $C$ en chaque point $B, K, L$ etc., $B O L$ étant le cercle de base et $D E F$ le cercle générateur de l'épicycloïde. Il prend une roue $D E F$ de centre $A$ avec des chevilles en $D, E, F$ «indéfiniment petites, \& perpendiculaires » au plan : d'après la démonstration précédente, « dans quelque position qu'on mettre la rouë $B O L$ dont les dents rencontrent les chevilles de l'autre rouë $D E F$, il y aura équilibre si les forces mouvantes appliquées aux circonférences de ces deux rouës sont égales ${ }^{155}$ ».

Il montre ensuite que la force appliquée à la roue $D E F$ est indépendante du nombre de dents qui agissent simultanément ${ }^{156}$. Puis il attribue à chaque cheville en $D, E, F$ etc. (Fig. 11) une forme cylindrique de rayon fini : ainsi, si $B V H$ est l'épicycloïde due au cercle générateur $D G M$, et si $B O P$ de centre $D$ est la coupe d'une cheville c'est-à-dire d'une dent de la roue $D G M$, chaque point de $B V H$ sera le centre d'un cercle (identique à $B O P$ ) et « $O N L$ qui touchera tous ces cercles, \& qui sera paralléle à l'Epicycloïde $[B V H]$, formera la figure de la dent de la rouë $B K^{157} »$.

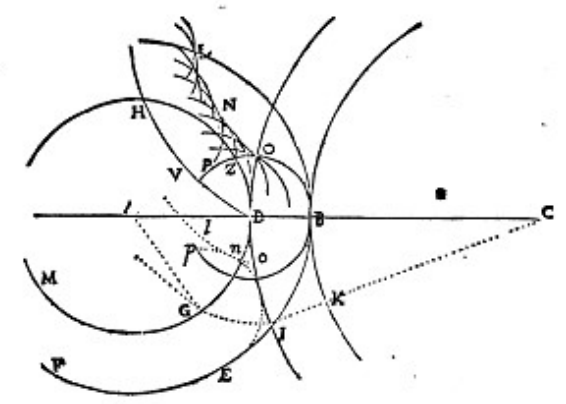

Fig. 11 : LA HiRE, Traité des Epicylö̈des, 1694, p. 59

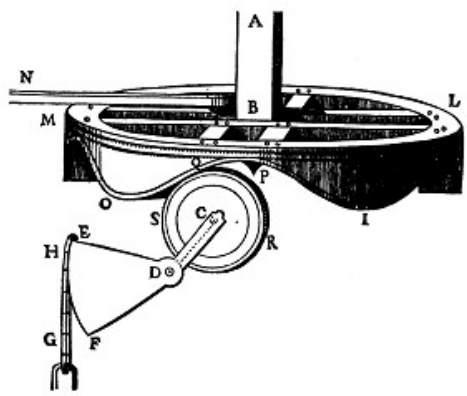

Fig. 12 : LA Hire, Traité des Epicylö̈des, 1694, p. 68

Par la suite, La Hire remplace la dent fixe $B O P$ ou $B Z P$ (Fig. 11) par « une roulette qui est mobile au tour de son centre $D$ sur un aissieu ou cheville » : il n'y aurait donc « d'autre frottement que celuy de la roulette $B Z P$ sur son aissieu ; car sa circonference s'appliquera par tout sans frotter sur la courbe de l'autre dent $O N L^{158}$ ». Enfin, il donne le mécanisme de la machine du château de Beaulieu ${ }^{159}$. LMOI (Fig. 12) est une roue actionnée par un levier en $N$ qui agit sur l'axe $A B$. La roue

${ }^{154}$ LA HiRE, «Traité des Epicyloïdes », 1694, p. 55.

155 Ibid., p. 56-57.

156 Ibid., p. 57.

157 Ibid., p. 58-60. La Hire précise que les dents de l'engrenage et du pignon ne doivent commencer à se rencontrer qu'en-dessous de la droite $A C$ (Fig. 11), autrement dit que le profil $O N L$ de la dent de $B K$ ne commence à agir contre la dent de la roue $D G M$ qu'au point $Z$ (lorsque $Z$ occupe dans le plan la place que $D$ occupait, les roues tournant respectivement de $B$ vers $K$ et de $D$ vers $G$ ) et il continue à agir sur la partie $Z P$ de la dent. La Hire écrit que si les dents «travaillent » au-dessus de $A C$ joignant les centres des roues alors « le frotement y est fort grand » et davantage que si la rencontre se fait en-dessous de $A C$. Il ajoute, qualitativement, que « les faces des dents qui se rencontrent en s'écartant l'une de l'autre ne se frottent qu'en s'échappant, ce qui n'apporte pas un empeschement sensible dans le mouvement; au lieu que lorsque le frottement se fait par la rencontre des parties qui rentrent l'une sur l'autre, l'empeschement au mouvement est fort considerable, \& c'est ce que l'on doit principalement éviter dans les machines ». Ibid., p. 60 et p. 67

${ }^{158}$ Ibid., p. 68.

159 FontenelLe, «Eloge de M. de La Hire», 1719, p. 84 rapporte que : «M. de la Hire trouva que ces dents [des roues] pour avoir toute la perfection possible devoient être en figure d'ondes formées par un arc d'Epicycloïde. Il fit executer son idée avec succés au Château de Beaulieu à 8 lieües de Paris dans une Machine à élever de l'eau. Il faut avoüer que cette idée n'a été executée que cette fois-là, une certaine fatalité veut qu'entre les inventions il y en ait peu d'utiles, \& entre les utiles peu de suivies ». 
a cinq dents ${ }^{160}$ qui agissent en passant sur deux « roulettes » $R S$ diamétralement opposées, chacune mobile autour de son essieu $C$, lequel tient à la pièce $C D E F$ mobile autour d'un essieu en $D$, essieu qui est fixé. Si la roue tourne de $O$ vers $I$, « la roulette descendra dans la rencontre de la partie $O Q$ de l'onde, \& elle remontra dans l'autre »; «lorsque [...] une [roulette] se trouve dans le fond ou creux de l'onde l'autre se trouvera sur le haut ». L'abaissement provoque la remontée d'un piston d'une pompe refoulante joint à la chaîne $H G$ qui permet une élévation d'eau, tandis que la roulette opposée qui remonte « ne fait aucun effort contre la rouë, car elle suit seulement la sinuosité de la dent» et n'est élevée que par le poids du piston et du système $D E F$ joint à la chaîne ${ }^{161}$. La détermination du profil des dents ou « ondes » repose alors sur des méthodes similaires à celles exposées ci-dessus ${ }^{162}$. La Hire applique ensuite ces constructions « aux ailes ou bras de l'arbre de moulins qui ont leur rouë verticale » qui actionnent des pistons ou des pilons, et montre que les profils de ces ailes sont épicycloïdaux ${ }^{163}$.

Du point de vue des principes mécaniques, La Hire s'appuie donc sur la loi du levier et sur un principe de statique semblable à celui de Descartes. Que La Hire recourt à ce dernier principe et non à un autre où interviendrait le temps ou les vitesses est peut-être lié à ce refus de faire entrer la «Physique » dans une science qui à ses yeux doit être fondée géométriquement; La Hire rejoindrait Descartes sur ce point. Sa méthode générale repose sur l'équilibre de deux roues qui agissent l'une sur l'autre, roues réduites à des leviers. L'équilibre revient à signifier une égalité entre les «efforts» transmis de la roue motrice à réceptrice. Dans la circonstance où les dents de la première roue ont un profil épicycloïdal, la force $X$ appliquée à la deuxième roue, et requise pour l'équilibre, égale celle exercée par la dent de la première ${ }^{164}$. Dans la partie mathématique du «Traité des Epicycloïdes », La Hire écrit donner « les quadratures des Epicycloïdes, tant de l'espace que de la ligne à la maniere des Anciens ${ }^{165}$ ", et Fontenelle remarque que La Hire «dans tous ses ouvrages de Mathematique [...] ne s'est presque jamais servi que de la Synthese, ou de la manière de démontrer des Anciens par des lignes \& des proportions de lignes ${ }^{166}$ ». La Hire exhibe l'épicycloïde comme la courbe qui répond à la condition requise d'un «mouvement égal » comme il l'écrit, autrement dit d'une force égale, mais il ne dévoile pas comment il parvient à cette courbe : plutôt qu'une analyse permettant à partir de cette condition de la déterminer, il livre une synthèse sans expliciter la voie prise pour son invention. C'est le reproche, entaché d'une suspicion de plagiat, qu'adresse Bernoulli à l'encontre de La Hire qui estime qu'il ne donne pas l'origine de sa découverte de la forme des dents, La Hire ne recourant pas, qui plus est, au calcul différentiel ${ }^{167}$.

${ }^{160}$ LA HIRE, «Traité des Epicyloïdes », 1694, p. 69 écrit qu’il faut un nombre de dents impair « afin qu'il y ait toûjours une des deux roulettes opposées qui travaille ».

${ }^{161}$ Ibid.

162 Ibid., p. 70-72. Ici, la construction de l'«onde » repose sur le mouvement de la roulette $R S$ (Fig. 12) sur une ligne horizontale (un diamètre de la roue $L M O I$ ) ce qui donne un profil cycloïdal.

163 Ibid., p. 72-77. La Hire montre que l'aile fixée à l'arbre du moulin qui par sa rotation la fait agir sur une roulette liée au bras rectiligne d'un piston ou d'un pilon a un profil épicycloïdal ; lorsque l'aile fixée à l'arbre est droite et qu'à son extrémité on place une roulette, cette dernière doit rouler sur une cyclö̈de liée au piston ou au pilon. La Hire, Traité de mécanique, 1695, p. 364-379 traite aussi de la machine du château de Beaulieu et du profil des ailes des moulins; il examine une machine supplémentaire, "qui sert à faire mouvoir plusieurs scies pour scier des pierres » en s'appuyant sur le même type de construction de courbes afin d'obtenir des actions continues de même intensité.

${ }^{164} \mathrm{Ou}$ la somme des forces exercées si plusieurs dents épicyclö̈dales agissent simultanément sur les dents du pignon.

165 LA Hire, «Traité des Epicyloïdes », 1694, « Préface » (non paginée).

166 Fontenelle, «Eloge de M. de La Hire », 1719, p. 88.

167 Voir Jean I Bernoulli à Leibniz du 8 (18) février 1698 dans LEIBNIZ, Sämtliche Schriften und Briefe, 2011, p. 734 et SERIS, Machine et communication, 1987, p. 187 : «Dissertationem de Epicycloidibus nondum vidi, sed aliquid de ipsarum usu ad figuras dentium etiam in ispo tractatu mechanico habet, inter alia constructionem alicujus rotae hoc modo dentatae omni notabili frictione carentis, quam se ipsum executione dedisse ait propre Lutetiam, cujus tamen primam inventionem $\mathrm{D}^{\text {no }}$ Desargues tribuit ; unde vero figuram dentium ad procurandum motum aequabilem didicerit, altum et silentium. Credebam equidem primo, nonnisi conjecturando voluisse divinare figuram debere esse cycloidalem, quod forsan hæc ipsi præ alia visa fuerit aptior, etenim ut modo dixi dissertationem de Epicycloidibus non vidi, neque in tractatu mechanico demonstrationem addit; postea vero ut rei certior fierem figuram debitam ex me ipso quæsivi, atque ex calculo comperi cycloidem vulgarem satisfacere, sed illam non solam, mamque (quod La Hirius non habet) et 


\subsubsection{Frottements solides, «force 》 des hommes et des animaux}

La Hire participe aux recherches de son temps sur les frottements solides et la quantification de la «force» des hommes et des animaux ${ }^{168}$. Nous soulignons ci-dessous que sa contribution n'aboutit pas à l'application aux machines de lois de frottements solides ou d'un antécédent du concept de travail, contrairement à ses confrères académiciens Parent et Amontons ${ }^{169}$.

Bien que le «Traité des Epicyclö̈des » évoque les frottements, il s'attache davantage à trouver la forme des dents des engrenages afin d'obtenir des mouvements réguliers. La Hire aborde les frottements solides en vérifiant des lois établies expérimentalement et en proposant un modèle mécanique pour celles-ci lors de la séance à l'Académie des sciences du 19 décembre 1699. Sur une table en bois «non polie », il dispose des morceaux de bois «non polis » dont « la superficie qui touchoit sur la table étoit de differente grandeur dans chacun comme dans la proportion 1. 2. 3. 4. 5. 6.7 et 8 ». Ces morceaux sont reliés à une « ficelle » horizontale parallèle à la table qui passe par une poulie "très bien tournée avec son petit axe», ficelle dont l'autre extrémité qui pend verticalement est attachée à un poids. La Hire charge chaque morceau de bois d'un poids de 2 livres $1 / 4$ et constate qu'il faut «le même poids ataché [1 livre 3/4] a l'autre extremité de la ficelle pour commencer a les faire couler sur la table ». Il réitère l'expérience avec deux morceaux de marbre non polis de 20 pouces et de 6 pouces de superficies, chacun pesant 4 livres $1 / 2$, qu'il pose sur une table en marbre non polie : «ils ont commencé a se mouvoir en coulant horisontalement sur la table » avec un même poids de 1 livre 5 once. La même expérience avec ces mêmes marbres sur une table «couverte d'eau » de telle sorte qu' «il y en avoit autant entre les deux Marbres que la Charge en pouvoit soutenir » montre que chaque marbre requiert le même poids pour être mis en mouvement, "mais que ce pois devoit étre un peu plus pesant que lors qu'il ny avoit rien entre deux [les morceaux de marbre et la table en marbre] comme dans l'experience precedente ${ }^{170} »$.

La Hire souligne que « ces experiences sont contre le sentiment commun » car « on a toujours cru que les frotemens étoient dans la raison des superficies touchantes la charge des superficies étant égales (sic) ${ }^{171} »$; il ajoute que lui-même conçoit, dans son Traité de mécanique, les frottements « dans la raison » des superficies de contact ${ }^{172}$. Dans ce Traité, La Hire écrit considérer les machines «sans avoir de corps, dont les parties peuvent empêcher l'effet, soit par leur pesanteur propre ou par le frotement qu'elles font les unes sur les autres », et qu'on peut « faire voir » comment diminuer le frottement dans les machines «quoy qu'on ne puisse pas donner les régles tres-certaines pour déterminer la quantité de frotement ${ }^{173} »$. Les travaux d'Amontons infléchissent vraisemblablement sa pensée : « $\mathrm{M}^{\mathrm{r}}$ Amontons aïant reconnu dans l'experience du poliment des Glaces de miroir que le frottement augmentoit dans la raison des charges et non pas des superficies, aussi tôt qu'il m'en eut averti j'en fus pleinement convaincu par la raisonnement Phisique et les experiences que j'ay

\footnotetext{
protracta et contracta idem præstant. Mirabar itaque quis Genius hunc hominem calculi nostri aliusve novae methodi omnino rudem et osorem in cognitionem harum figurarum deduxisset. Ast postquam Romeri inventum esse ex Te cognovi, cesso mirari ». Voir aussi LEIBNIZ, Sämtliche Schriften und Briefe, 2004, p. 343, p. 365 : L’Hospital écrit à Leibniz le 25 avril 1695 que dans le «Traité des Epicycloïdes » il n’y a « rien de nouveau, sinon les demonstrations qui sont à la maniere des anciens »; Leibniz écrit à L’Hospital le 13 (23) mai 1695 trouver " fort bon que Monsieur de la Hire demonstre les nouvelles découvertes à la façon des anciens Geometres et on luy en aura de l'obligation, parce qu'il rend ainsi temoignage de la verité. Mais il aura souvent besoin de beaucoup de paroles ».

168 Pour une contextualisation de ces recherches et leur importance en particulier au sein de l'Académie royale des sciences, voir notamment SERIS, Machine et communication, 1987, p. 159-210 et FONTENEAU, Développements précoces du concept de travail, 2011, p. 35-260.

${ }_{169}$ Pour cette conclusion concernant le travail chez Parent et Amontons, nous nous appuierons essentiellement sur les analyses approfondies de FONTENEAU, Développements précoces du concept de travail, 2011.

${ }^{170}$ LA HIRE, «Experiences et Observations », 1699, f. 570r-570v.

${ }^{171}$ Ibid., f. 571 r.

${ }^{172}$ C'est manifestement le cas pour le contact entre le pas d'un écrou et celui d'une vis, voir La Hire, Traité de mécanique, 1695 , p. 325.

173 Ibid., p. 7.
} 
faites ensuite et que je viens de rapporter le font manifestement connoitre ${ }^{174} »$. Les Procès-verbaux de l'Académie des sciences contiennent après l'intervention de La Hire un mémoire d'Amontons portant sur ce sujet, avec cette précision que « $\mathrm{M}^{\mathrm{r}}$ Amontons [...] le premier avoit donné occasion d'examiner particulierement cette Matiere ${ }^{175} »$. Le mémoire lu par La Hire ne sera pas publié contrairement à celui d'Amontons ${ }^{176}$; l'Histoire de l'Académie pour l'année 1699 donne un résumé des réflexions de La Hire sur les causes physiques des frottements tirées de ses « Experiences et Observations $^{177}{ }^{1}$.

Ainsi, pour deux corps en contact par des "parties molles et flexibles », comme c'est le cas pour des morceaux de bois, «ces parties » peuvent être assimilées à des "petits Ressorts » qui ploient par la charge et qui seront d'autant plus bandés que « la charge sera plus grande ou bien à proportion de la quantité qu'il y en aura qui supportera la même charge » : "1000 Ressorts pour soutenir le poids d'une livre [...] seroient une fois plus bandés que s'il y en avoir deux mile (sic) ce qui est dans la raison des superficies, supposant qu'il y en a egalement dans des parties egales de la meme superficie du même corps». Ces 1000 ressorts feront «autant de resistance» que les 2000, résistance qui est donc « en raison des poids » et non du nombre de «Ressorts », autrement dit des superficies ${ }^{178}$. Pour ce qui est des corps dont « les parties qui touchent sont dures et incapables de Ressort » comme les marbres, «leurs parties [...] sont engagées l'une dans l'autre ». Si elles sont dures au point de ne pas rompre, elles peuvent «se dégager les unes des autres en s'elevant elles ne se rompront tous au plus que dans leurs extremitez, et ce degagement des parties deppendant seulement de la pesanteur du corps et non pas du nombre des parties engagées ou des superficies ce qui est la méme chose, puisque, le corps doit s'elever pour se dégager la resistance qui viendra de ce frotement sera toujours dans la raison du poids et non dans la raison des parties engagées ou des superficies». Toutes choses égales par ailleurs, l'eau modifie le degré d'engagement des « inégalités» du corps du dessous sur celui du dessous, ces «inégalités » n'entrant "pas si avant»; ceci ne change pas le fait que «l'Effort pour le [le corps du dessus] faire marcher sera toujours dans la raison de sa pesanteur ${ }^{179} »$.

A partir de ses travaux expérimentaux, Amontons détermine, à travers un exemple, un effort de traction pour mettre en mouvement un corps compte-tenu des frottements, puis livre une série de « règles" pour le calcul des frottements et des forces mouvantes applicables à des machines «composées » en mouvement ${ }^{180}$. Par ailleurs, dans le cadre de quatre mémoires portant sur une "Nouvelle Statique avec frotemens », Parent établit des « regles» pour trouver les forces qui équilibrent des charges sur des machines dont les parties frottent les unes sur les autres ; ces travaux concernent avant tout la statique et les machines simples, Parent se plaçant à la limite de la rupture de leur équilibre ${ }^{181}$. Pour sa part, La Hire ne propose pas d'applications de « règles » de frottements

${ }^{174}$ LA HiRE, « Experiences et Observations », 1699, f. 571r.

175 AMONTONS, «De la resistance causée dans les Machines », 1699, f. 573 r.

176 Ibid., 1701.

177 Voir FOnTENELLE, «Sur le frottemens des machines », 1701, p. 104-109. NOLLET, Leçons de physique expérimentale, 1743, p. 235-236 évoque ce résumé lorsqu'il aborde la question des frottements.

178 LA HIRE, «Experiences et Observations », 1699, f. 571r-571v.

${ }^{179} \mathrm{Ibid}$., f. 571v-572r. Voir le résumé de FONTENELLE, «Sur le frottemens des machines », 1701, p. 104-105. La HIRE, "Experiences et Observations », 1699, f. 572v, précise qu’il est des cas où les surfaces de contact entrent en ligne de compte dans la mesure des frottements. Ainsi, en huilant ces surfaces, l'air est chassé d'entre les pièces qui se touchent et il ne s'y en insinue pas car «ses particules sont plus Grossieres que celle de l'eau ou de l'huile ». Par conséquent, il n'y a pas d'air pour contrebalancer l'action de l'air extérieur à ces pièces, et « par Consequent la charge des Corps touchans ou coulans l'un sur l'autre dans ce cas doit etre considerée par rapport a leur Superficie, qui est celle de l'atmosphere sur chacun en particulier jointe avec la propre pesanteur du Corps ce qui fera le frotement a tres peu pres dans la Raison de ces Superficies ».

180 Amontons, «De la resistance causée dans les Machines », 1701, p. 207-217. Voir SERIS, Machine et communication, 1987, p. 192-210.

181 Voir PARENT, « Nouvelle Statique avec frotemens », 1706, « Second mémoire », 1706, «Troisieme Mémoire », 1706, et «Quatiéme Mémoire », 1714. Parent ne s'attache pas dans ces études aux charges et aux agents en mouvement. 
aux machines et ses travaux sur ce sujet consistent essentiellement à vérifier expérimentalement des lois établis par d'autres et à leur donner une interprétation physique.

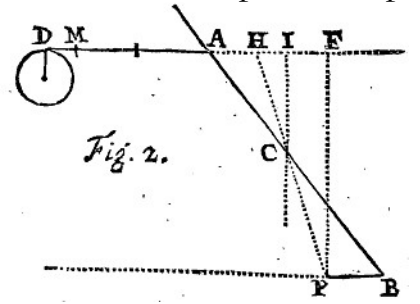

Fig. 13 : LA HIRE, «Examen de la force de l'homme», 1702, p. 159

La contribution de La Hire à la détermination de "la force de l'homme » repose sur des considérations statiques. Pour évaluer «l'effort de l'homme pour tirer ou pour pousser horizontalement", il suppose une force appliquée à la manivelle d'un rouleau $D$ (Fig. 13) autour duquel s'enroule une corde qui soutient un poids sans avoir " égard aux frottemens de l'axe du rouleau, ni à la difficulté que la corde peut avoir à se ploïer ${ }^{182}{ }$. $A M$ représente la ligne allant des épaules aux mains et $H P$ le corps de l'homme assimilable à « un levier ou verge droite $P C H$ »de point d'appui $P$ où se trouvent les pieds. La force exercée sur la manivelle « dépend de la seule pesanteur du corps » appliquée au point $C$ «le centre de gravité qui est a peu près la hauteur du nombril au-dedans du corps »; cette pesanteur est prise égale à 140 livres. La Hire cherche alors « quel effort la pésanteur du corps en $C$ avec sa direction naturelle, peut faire sur la manivelle selon la direction horizontale $D H »$. Avec $P H$ de $« 140$ parties » ${ }^{183}$ et $P C$ de 80 , puisque le poids en $C$ vaut 140 livres, son « effort» sera en $H$ de 80 livres dirigées verticalement d'après la loi du levier. En traçant $P F$ normale à $A M$, le rapport du poids en $H$ à « l'effort » sur la manivelle égale $\frac{P F}{H F}$. Avec $\widehat{P H F}=70^{\circ}, \frac{P F}{H F} \cong 3$ et «l'effort» sur la manivelle vaut environ 27 livres. Cet «effort » est identique pour pousser horizontalement avec les bras ou pour tirer horizontalement avec une corde fixée aux épaules ou à la taille pour une même inclinaison du corps ${ }^{184}$. Lorsque La Hire compare la « force » des chevaux à celle des hommes lors de la traction, il précise que « comme elle ne dépend pas entierement de leur pesanteur, comme celle des hommes, mais principalement des muscles de leur corps \& de la disposition générale de ses parties qui ont un très-grand avantage pour pousser en avant, on doit se contenter de l'expérience commune qu'on a, qu'un cheval tire horizontalement autant que sept hommes ; \& ainsi un cheval ne peut tirer horizontalement qu'un peu moins de 200. 1. », soit sept fois 27 livres ${ }^{185}$. Cette proportion de 7 à 1 est le fruit d'observations et d'expérimentations menées en 1668 par des académiciens des sciences lors de la construction de l'Observatoire de Paris ${ }^{186}$.

Paul Joseph Barthez, auteur de l'article « Force des animaux » de l’Encyclopédie considère que « le premier auteur qui ait examiné la force de l'homme avec quelque précision, \& qui l'ait comparée avec celle des autres animaux, c'est sans doute M. de la Hire, dont l'écrit sur ce sujet est imprimé parmi les mémoires de l'académie des Sciences, année $1699^{187} »$. Barthez résume le mémoire de La Hire ainsi que des passages du Course of Experimental Philosophy de John Théophilus Desaguliers, lequel

${ }^{182}$ LA HIRE, «Examen de la force de l'homme », 1702, p. 157. La Hire précise que la distance à l'axe du rouleau jusqu'au coude de la manivelle égale le rayon du rouleau « afin de comparer la force appliquée sans aucune augmentation de la part de la machine ». La quantification de l'effort à fournir pour "plö̈er » des cordages fait partie des recherches expérimentales menées par AMONTONS, «De la resistance causée dans les machines », 1701, lequel, comme pour les frottements solides, donne des « regles pour le calcul de la roideur des Cordes dans les Machines ».

${ }^{183}$ LA HIRE, «Examen de la force de l'homme », 1702, p. 159 indique de manière erronée « 240 parties ».

184 Ibid., p. $157-160$

185 Ibid., p. 161.

186 Voir les Procès-verbaux de l'Académie royale des sciences, t. 3 , 3 juillet 1668, f. 77r-78r pour cette proportion établie sur le chantier de l'Observatoire.

187 BARTHEZ, «Force des animaux », 1757, p. 120 b. 
traduit intégralement et commente le texte de La Hire ${ }^{188}$. Avec un mécanisme semblable à celui cidessus de La Hire, il est notable que, pour sa part, Desaguliers quantifie la « force » en prenant en compte la durée du travail et de la vitesse d'élévation du fardeau : " he shall not have above 30lb Weight acting against him, if $\mathrm{h}$ is to work ten Hours a Day, and raise the Weight about 3 Foot and an half in a Second, which is the common Velocity that a Horse draws with ${ }^{189} »$. Dès 1699, ce type de quantification figure chez Amontons qui, contrairement à La Hire, ne recourt pas seulement à un équivalent statique, à savoir le poids qu'est capable de soutenir un agent, mais intègre le temps d'exercice de l'effort et la distance parcourue lors de celui-ci. La notion introduite dénommée par Amontons "puissance continuelle» est à même d'entrer dans une comparaison des effets réalisables par des machines, des animaux et des hommes en considérant pour ces derniers la durée journalière du travail effectif. Pour Amontons, «l'effet ça n'est plus ce qu'on peut soutenir ; ça n'est plus ce qu'on peut soutenir en le déplaçant tout en croyant que la statique puisse donner l'exacte mesure de la force nécessaire [...] ; l'effet c'est désormais, et dans une double évolution, non seulement la force dynamique nécessaire mais la comptabilisation de ce mouvement même, distance ou vitesse »; ainsi l'effet n'est pas simplement un poids à soutenir mais «intègre la considération de la vitesse d'exécution » et cette force n'est plus « une force-pour-soutenir » mais une «force-pour-mouvoir ${ }^{190} »$. Dans un autre mémoire, La Hire quantifie l'effort de chevaux qui tirent un bateau en considérant la durée pendant laquelle ils meuvent cette charge sur une certaine distance, mais il semble qu'il s'agit là de la seule publication développant une telle mesure ${ }^{191}$.

Evaluer un effort est avant tout, pour La Hire, déterminer la charge capable d'équilibrer ; mesurer est avant tout peser par le biais de la loi du levier et La Hire ne développe pas d'antécédent du concept de travail mécanique contrairement à Amontons. Par ailleurs, il travaille aux perfectionnements de machines comme l'illustrent ses recherches sur les profils des dents des engrenages, mais il n'élabore pas les instruments permettant de quantifier ce que Parent nomme « la plus grande perfection possible des machines » : Parent calcule ce qu'il appelle « le plus grand effet possible » d'une machine en fonction d'un travail moteur, le savant tenant compte des contraintes et de la structure de l'engin qui imposent des limites à son efficacité. Le perfectionnement auquel vise La Hire consiste essentiellement à appliquer la géométrie aux arts et la solution apportée à un problème de nature physique - avoir un mécanisme au mouvement continu - ressortit avant toute chose à la géométrie : d'une part, La Hire ne prend pas en compte les frottements solides de ses engrenages et, d'autre part, contrairement à Parent, il ne développe pas une réflexion qui le conduirait, à partir de la quantification de l'effet de sa machine à s'interroger sur l'optimisation de son fonctionnement ${ }^{192}$. En ce sens, la science appliquée de La Hire semblerait à la fois s'enraciner dans une science de l'équilibre, et son approche pour améliorer le fonctionnement de machines se réduire avant tout à l'usage/l'application de la géométrie.

${ }^{188}$ Desaguliers, A Course of Experimental Philosophy, 1745, p. 253-257 et p. 276-283.

189 Ibid., p. 254. Avec 1 1. en France entre 1/11 e et 1/12 e plus grande qu'en Angleterre, voir DeSAGULIERS, Cours de physique expérimentale, 1751, p. 285.

${ }^{190}$ FONTENEAU, Développements précoces du concept de travail, 2011, p. 166-168. Les paramètres utilisés par Amontons pour évaluer un effet sont "représentatifs du travail accompli» et "également physiologiquement représentatifs de la fatigue " de l'ouvrier ou des animaux (p. 168). Pour une analyse détaillée des écrits d'Amontons en lien avec l'élaboration d'un antécédent d'un concept de travail ou de puissance mécanique, voir ibid., et SERIS, Macbine et communication, 1987.

191 Voir LA HiRE, «Examen de la force necessaire », 1704 et FONTENEAU, Développements précoces du concept de travail, 2011, p. 128-137 pour un examen de ce mémoire.

192 Ce que fait Parent pour une machine hydraulique. Il quantifie un effet par le produit d'un poids avec sa vitesse. Parent obtient une expression entre le poids équilibré $(\mathrm{P})$ par le fluide via cette machine en prenant en compte la vitesse du fluide - ce qui correspond à la force motrice disponible - et le poids soulevé (p) à une certaine vitesse. En considérant que ce dernier poids est une variable, l'annulation de sa différentielle conduit à une relation entre $\mathrm{p}$ et $\mathrm{P}$ qui donne ainsi le plus grand effet dont est susceptible la machine en fonction de P et de la vitesse du fluide. Voir PARENT, «Sur la plus grande perfection possible des Machines », 1706. Sur ce mémoire, voir SERIS, Machines et communication, 1987, p. 285-298 et FONTENEAU, Développements précoces du concept de travail, 2011, p. 203-211. 


\subsection{Cinématique et dynamique}

\subsubsection{Cinématique galiléenne et dynamique}

Le Traité de mécanique de La Hire aborde le choc au moins de deux manières et, dans les deux cas, en se référant essentiellement à Mariotte. Ainsi, ce livre contient des règles du choc pour les corps parfaitement durs et parfaitement élastiques, en énonçant une « regle générale » pour ces derniers qui revient celle de Mariotte qui est mentionné dans cet exposée ${ }^{193}$. C'est aussi manifestement sur Mariotte que La Hire s'appuie concernant les déterminations des centres de percussion et d'oscillation de pendules composés ${ }^{194}$.

La Hire énonce la loi de chute libre de Galilée et la démontre à l'occasion d'un examen du mouvement sur des plans inclinés ; il dépasse alors le cadre des propositions cinématiques telles qu'on les rencontre chez Galilée en faisant intervenir des forces ou «puissances » productrices de vitesses ${ }^{195}$. Galilée cherche comment évolue ce qu'il nomme l'« impeto » d'un corps sur un plan incliné, sachant que «les moments » où «les vitesses » d'un mobile varient selon les différentes inclinaisons du plan et, ainsi, que "l'impeto, la puissance, l'énergie ou [...] le moment de descente sont diminués dans le mobile par le plan sur lequel il prend appui et descend ${ }^{196} »$. Ce «moment de descente » égale la « résistance ou plus petite force » nécessaire pour arrêter le mobile. Soit $G$ sur un plan incliné de longueur $F A$ de hauteur $F C$ retenu par un contrepoids $H$, la statique donne $\frac{H}{G}=$ $\frac{F C}{F A}$ et $H$ mesure le «moment de descente partiel ${ }^{197} »$ de $G$ sur le plan et on a une relation du type $\frac{\text { Impeto ou moment de descente partiel }(G)}{\text { Impeto ou moment de descente total }(G)}=\frac{H}{G}=\frac{F C}{F A} 98$. La proportion vaut pour des mobiles et Galilée écrit que «tels auront été les impeto dans les débuts du mouvement, tels seront proportionnellement les degrés de vitesse acquis pendant le même temps, puisque les uns et les autres croissent en même temps avec la même proportion ${ }^{199} »$. Manifestement, le savant s'en tient ici à une mise en parallèle entre d'un côté le rapport des "moments" et, de l'autre, celui des vitesses,

193 LA HIRE, Traité de mécanique, 1695, p. 384-397. Cette « regle » consiste à considérer les corps dits «à ressorts » parfaitement durs lors de la phase de compression de telle sorte qu'ils soient animés d'une vitesse commune. Il faut ensuite diviser la «somme » des vitesses initiales (en comptées algébriquement) en proportion inverse de poids pour trouver les vitesses acquises par chaque corps du seul fait de l'action de leur « ressort» propre. Ces deux vitesses additionnées ou soustraites donnent celles des mobiles après le choc. La Hire écrit que «M. Mariotte donne la maniére de trouver ces vitesses par les divisions d'une ligne » et résume la construction géométrique des vitesses finales des corps. Pour cette règle, voir MARIOTTE, Traitté de la percussion, 1673, p. 115-119, et p. 90-97 pour la justification du partage de la vitesse respective en proportion inverse des masses.

${ }^{194}$ La Hire note que deux poids qui rencontrent les plateaux d'une balance de telle sorte que leurs quantités de mouvement soient en proportion inverse des bras s'équilibrent : en supposant ces poids liés entre eux par une verge, le point d'appui de la balance définit le lieu du centre de percussion du système composé par les deux corps; comme l'écrit Mariotte, il s'agit du point au niveau duquel il se fait le « plus d'impression par le chocq » ou encore celui de part et d'autre duquel les quantités de mouvements des corps sont égales. Cette dernière proportion permet de déterminer le centre de percussion identique au centre d'oscillation pour un pendule chargé de poids ; la distance entre le point de suspension d'un tel pendule composé et ce centre d'oscillation définit la longueur d'un pendule simple qui lui est isochrone. Voir LA HIRE, Traité de mécanique, 1695, p. 404-407 et MARIOTTE, Traitté de la percussion, 1673, p. 258-281. La Hire mentionne aussi Huygens pour l'identité des centres d'oscillation et de percussion pour un pendule composé, mais sa démonstration suit davantage les raisonnements de Mariotte. Sur les travaux sur les centres d'oscillation aux $17^{\mathrm{e}}$ et $18^{\mathrm{e}}$ siècles et les démonstrations de Huygens et de Mariotte, voir VILAIN, «La question du centre d'oscillation », 2000, p. 21-51 et p. 439-466.

${ }^{195}$ LA HiRe, Traité de mécanique, 1695, p. 382-384 et p. 408-421.

196 GALILEE, Discours concernant deux sciences nouvelles, 1995, p. 146 ; GALILEI, Discorsi e dimostrazioni matematiche, 1898, p. 215.

${ }^{197}$ Pour le « momento parziale », voir GALILEI, Discorsi e dimostrazioni matematiche, 1898, p. 217. Pour les différents sens du mot «momento » chez Galilée, voir GALLuZZI, Momento. Studi galileiani, 1979.

198 GALILEI, Discorsi e dimostrazioni matematiche, 1898, p. 217.

199 GALILEE, Discours concernant deux sciences nowvelles, 1995, p. 148 ; GALILEI, Discorsi e dimostrazioni matematiche, 1898, p. 218. 
sans établir de lien causal ${ }^{200}$. Un tel lien est manifeste chez Evangelista Torricelli et, par la suite, dans la deuxième moitié du $17^{\mathrm{e}}$ siècle où le "moment" de Galilée se voit assimilé à une force produisant une variation de vitesse au cours du temps ${ }^{201}$.

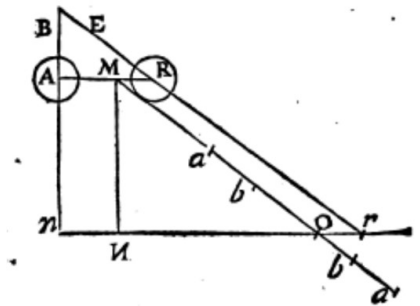

Fig. 14 : LA HiRE, Traité de mécanique, 1695, p. 408

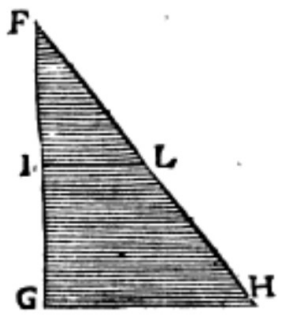

Fig. 15 : LA HiRE, Traité de mécanique, 1695, p. 410

La Hire, qui à l'occasion de propositions de chute libre sur les plans inclinés examine «ce que Galilée a expliqué fort au long dans son troisiéme dialogue du mouvement ${ }^{202}$ », s'inscrit dans un tel contexte de naissance de la dynamique. Il considère un même poids $A$ (Fig. 14) en $A$ et posé sur $M O$ en $R$ : avec la « puissance $B$ » qui « soutient» $A$ et la « puissance $E$ » qui le « retient» sur $M O$, il obtient à l'instar de Galilée ci-dessus $\frac{E}{B}=\frac{M N}{M O} 203$. Il considère ensuite $A$ et $R$ comme « deux points [...] sans aucune pesanteur \& [...] poussés par les puissances $B \& E$ ». En des temps égaux, ces puissances font parcourir à $A$ et $R$ « des espaces qui sont entr'eux [...] comme ces puissances » et ce en agissant " par des coups répétés dans des instans égaux \& indéfiniment petits 》. Dans $F G H$ (Fig. 15) «les parties égales \& indéfiniment petites du côté $F G$ du triangle, représenteront les instans égaux ». Dans «un premier instant» un «corps parcourt la premiere ordonnée avec un degré de vitesse uniforme», dans un deuxième instant «il reçoit un nouveau degré de vitesse pardessus le premier » et égal au premier, et « il parcourt la seconde ordonnée avec deux degrez de vitesse » etc. ; les ordonnées correspondant aux lignes parallèles à $I L$ et le rapport de deux d'entre elles égale le rapport des vitesses auxquelles elles sont associées, car « on suppose que dans chaque instant le corps se meut d'un mouvement uniforme avec sa vitesse particuliere ». Puis, si $F I$ et $F G$ représentent cents et deux cents instants, « il est évident que la somme des espaces parcourus dans les cent premiers instans sera à la somme des espaces parcourus dans les deux cens premiers instans, comme l'espace du triangle FIL à l'espace du triangle FGH »; ces « espaces » sont « dans la raison des quarrés des temps » car les vitesses acquises qui correspondent aux lignes $I L$ sont aussi proportionnelles aux durées.

Comme chez Galilée, on retrouve le passage d'une proportion statique à une proportion cinématique : le rapport de ce que La Hire nomme les « puissances » $E$ et $B$ qui équilibrent est celui des « degrés de vitesses » acquis le long de $M N$ et $M O$ par les deux mobiles ${ }^{204}$. Mais la « pesanteur» chez La Hire n'est ici qu'un cas particulier de force ou de "puissance ${ }^{205 "}$ et ce que La Hire nomme

\footnotetext{
${ }^{200}$ Sur cette absence de lien causal, voir GALLuZZI, Momento. Studi galileiani, 1979, p. 403 et p. 405, et DE GANDT, Force and Geometry, 1995, p. 105-107. Concernant Torricelli et le principe fondamental de la dynamique, voir WeSTFALL, Force in Newton's physics, 1971, p. 125-138, DE GANDT, Force and Geometry, 1995, p. 102-105 et DE GANDT, "L'analyse de la percussion », 1989, p. 57-77.

${ }^{201}$ Mentionnons en particulier des travaux de John Wallis de 1670-1671, de Claude François Milliet Dechales de 1682 et de Pierre Varignon de 1692-1693. Voir pour une analyse de ceux-ci, voir SCHMIT, «Rapports entre équilibre et dynamique », 2014.

${ }^{202}$ LA HiRe, Traité de mécanique, 1695, p. 409. La Hire se réfère ici aux Discorsi de Galilée, ce «troisiéme dialogue » correspondant à la troisième journée portant sur le «mouvement local». LA HiRE, Traité de mécanique, 1695, p. 418 montre notamment que les temps mis par un corps à parcourir des plans de même hauteur mais différemment inclinés sont proportionnels aux longueurs, que le corps en mouvement sur un plan ou en chute libre acquiert pour une même hauteur la même vitesse. Pour ces propositions, voir GALILEI, Discorsi e dimostrazioni matematiche, 1898, p. 215-219.

${ }^{203}$ LA HIRE, Traité de mécanique, 1695, p. 296-297 et p. 408-421.

204 Ibid., p. 412.

${ }^{205}$ Cette généralité se trouve déjà chez Wallis et chez Varignon, voir SCHMIT, « Rapports entre équilibre et dynamique », 2014.
} 
ici «points » correspondent à des points matériels voire des masses ${ }^{206}$. Figure aussi un lien causal entre une "puissance" et l'acquisition par un corps d'un "degré de vitesse", cette action étant instantanée : les "instants égaux \& indéfiniment petits » le long de $F G$ ne représentent pas tant les durées d'exercice des puissances que la fréquence régulière des « $\operatorname{coups}^{207}$ ». Par ailleurs, pour La Hire à « chaque instant le corps se meut d'un mouvement uniforme avec sa vitesse particuliere » et c'est comme si, au lieu d'un même « coup » d'intensité $P$ et donnant une vitesse $V$ lors du premier instant et répété à chaque instant, une nouvelle force $2 P$ agissait au deuxième instant donnant la vitesse $2 V$ etc. et à l'intant $n$, comme si $n P$ agissait donnant $n V$. Or, en considérant « les instans égaux », d'un instant à l'autre, le rapport des « vitesses particulieres » dite " uniformes » est comme celui des espaces franchis par ces vitesses à savoir des longueurs $I L$. Le procédé permet de tracer des lignes dont l'agrégat dessine un triangle assimilé à l'espace total franchi lors d'une chute : pour La Hire, il semble « évident» que la hauteur de chute s'identifie à la surface d'un triangle, mais il n'explicite pas cette identification ${ }^{208}$. Ces procédés basés sur une action instantanée et la sommation de "vitesses particulieres" identifiées à des segments sont identiques à ceux à l'œuvre chez Varignon, Nicolas Guisnée et Isaac Barrow ${ }^{209}$.

Tous ces éléments inscrivent alors la pratique de La Hire dans le prolongement des travaux de Galilée, en leur donnant indéniablement une teneur dynamique, mais aussi dans un moment antérieur à une conceptualisation des vitesses et des accélérations instantanées, et à la formulation analytique de celles-ci ${ }^{210}$. À la suite de ces propositions, La Hire démontre que le temps de chute mis par un corps le long d'une "cycloïde renversée» pour arriver au sommet de la courbe ne dépend pas de l'endroit où le corps est lâché, le savant mentionnant alors les travaux de Huygens sur la question ${ }^{211}$. L'examen de recherches de La Hire sur les cyclö̈des et leur comparaison avec celles de Varignon montre qu'il participe aux travaux de cinématique de la fin du $17^{\mathrm{e}}$ siècle qui, souvent sous la forme de problèmes que se posent des savants dans des journaux, consistent à déterminer la nature de courbes le long desquelles se meuvent des mobiles soumis à certaines conditions $^{212}$. Malgré tout, La Hire garde une approche qui lui est propre et, en particulier, ne fait pas usage du calcul différentiel et intégral.

\subsubsection{La Hire, Varignon: calcul différentiel et chute libre le long d'une cycloüde}

Dans les années 1693 à 1696, des travaux du Marquis de L’Hospital, de Pierre Varignon, de Joseph Sauveur ou encore de Thomas Frantet de Lagny contribuent à l'essor du calcul leibnizien au sein de l'Académie royale des sciences ${ }^{213}$. Le mémoire du 23 février 1697 de La Hire est une réponse à cette évolution. L'auteur remarque que « ces derniers temps on a commencé a considerer les courbes comme des figures Polygones dont les côtez estoient si petits qu'ils ne pouvoit y avoir aucune difference sensible entre l'arc de la courbe et sa corde ou côté Polygone », moyen par lequel « on a fait une infinité de decouvertes tres curieuses dans ce genre de Geometrie ». Mais il évoque

\footnotetext{
206 Varignon appelle «masses» ce que La Hire nomme ici "points». Voir SCHMIT, «Rapports entre équilibre et dynamique », 2014.

207 Voir SCHMIT, «Rapports entre équilibre et dynamique », 2014 pour une telle conception de l'action chez Nicolas Guisnée, élève de Varignon, et chez ce dernier.

${ }^{208}$ Galilée n'explicite pas davantage cette identification, voir GALILEI, Discorsi e dimostrazioni matematiche, 1898, p. 208209 et les analyses de DE GANDT, Force and Geometry, 1995, p. 107-109.

209 Voir SCHMIT, « Rapports entre équilibre et dynamique », 2014.

${ }^{210}$ Pour ces concepts et l'algorithmisation chez Varignon avec le calcul différentiel et intégral leibnizien en 1698-1700, voir BLAY, La naissance de la mécanique analytique, 1992, p. 153-221.

${ }^{211}$ LA HIRE, Traité de mécanique, 1695, p. 421-424.

212 Ainsi, il s'agit de trouver la courbe sur laquelle un corps pesant s'approche uniformément de l'horizon (courbe isochrone) ou d'un point (isochrone paracentrique), celle de plus rapide descente (brachystochrone), ou encore celle sur laquelle un corps presse à chaque instant également la surface. Voir BLAY, La naissance de la mécanique analytique, 1992, p. 63-109.

213 Pour ce contexte, voir BLAY, La naissance de la mécanique analytique, 1992.
} 
aussi les « suppositions » faites dans ce calcul « qui laissent toujours quelque doute dans l'Esprit de ceux qui ne veulent rien accorder que ce qui est démontré dans la rigueur Geometrique », en particulier que « les tangentes des courbes sont aussi bien reputées égales à l'arc de la courbe que la corde de cet arc ». Pour sa part, La Hire donne un exemple illustrant qu'« il est difficile de ne pas tomber dans l'erreur », à savoir la supposition admise par ceux qui recourent au calcul que la corde d'un arc de cercle « indéfiniment petit ou aussi petit qu'on le puisse imaginer » égale cet $\operatorname{arc}^{214}$.

Ainsi, un corps qui tombe verticalement de $A$ vers $B$ (Fig. 16) sans vitesse initiale, avec $A B=$ 15 pieds en 1 seconde, met le même temps s'il se meut sur la corde $E B$ en allant de $E$ sans vitesse vers $B$. En prenant la corde $B D$ «indéfiniment petite », le mobile met encore le même temps et, par hypothèse, puisque « il n'y a point de difference sensible entre cette corde $B D$, et son arc », il parcourt aussi l'arc $B D$ en 1 seconde. En supposant le corps accroché à un pendule attaché en $C$, il emploie 1 seconde à parcourir $B D$. Autrement dit, un pendule de 7 pieds $1 / 2$ de longueur fait une «vibration» en 2 secondes de temps or l'expérience montre qu'un pendule qui employe deux seconde pour une « vibration » est long de 12 pieds et environ 3 pouces ${ }^{215}$ : « une difference trop grande pour l'attribuer a quelqu'autre chose qu'a la supposition qu'on a faite, que le corps tombe par l'arc infiniment petit et par sa corde dans un même espace de temps ${ }^{216} »$.

La question du manque de rigueur des fondements du calcul différentiel et le fait qu'il puisse conduire à des erreurs sont les deux angles d'attaque de Michel Rolle dans ses polémiques avec Varignon en 1700-1701 et par la suite avec Joseph Saurin en 1702-1705 $5^{217}$. La Hire est nommé en 1701 et 1705 membre de deux commissions devant donner un jugement officiel aux débats RolleVarignon et Rolle-Saurin ${ }^{218}$; le résumé de son intervention de février 1697 pourrait suggérer qu'il est à la fois juge et partie.

De fait, ceux qui promeuvent le calcul différentiel et intégral leibnizien voient en La Hire un opposant à celui-ci. Selon L'Hospital, La Hire donne dans son traité des épicycloïdes des « demonstrations [...] à la manière des anciens, ce qui les rend longues et ennuyeuses ${ }^{219}$ » et pour Leibniz, il prend la peine dans ce livre de « reduire en demonstrations à la façon des anciens, ce que nous découvrons aisement par nos Methodes ${ }^{220}$ ", ou bien encore il « adjoute à des méditations de nostre Analyse une Synthese à la façon des anciens $»^{221}$. Varignon écrit à Jean Bernoulli avoir « desja soutenu d'assauts pour eux [les infiniment petits] contre certains mathématiciens du vieux stile, qui chagrins de voir que par ce calcul les jeunes gens les attrapent et mêmes les passent, font tout ce qu'ils peuvent pour le décrier ${ }^{222}$ », des propos que Bernoulli rapporte à Leibniz en précisant qu'il

${ }^{214}$ LA HiRE, « Remarque sur l’usage », 1697, f. 23r-24r.

${ }^{215} \mathrm{La}$ « vibration » correspond ici à une demi-période d'oscillation du pendule.

${ }^{216}$ LA HIRE, «Remarque sur l'usage », 1697, f. 25r-25v. BLAY, La naissance de la mécanique analytique, 1992, p. 38-41 analyse une partie de ce mémoire. Comme il le remarque, le problème revient à chercher la courbe telle qu'un corps sans vitesse initiale partant de $O$ arrive en $P$ (Fig. 16) dans le même temps qu'il parcourait la corde $O P$; l'arc de cercle n'est pas la solution, mais il s'agit d'un arc de lemniscate de Bernoulli. Le raisonnement et l'expérience mécaniques de La Hire ne mettent pas en lumière que l'identité entre corde et l'arc résulte d'un passage à la limite.

217 Voir notamment BLAY, «Deux moments de la critique » 1986, MANCUSO, "The Metaphysics of the Calculus », 1989, et BLAY, La naissance de la mécanique analytique, 1992, p. 42-62.

218 Voir Procès-verbaux de l'Académie royale des sciences, 1701, séance du 3 septembre, f. 335v : «comme la dispute des Infinimt. Petits trainoit trop en longueur, $\mathrm{M}^{\mathrm{r}}$. l'Abbé Bignon à nommé pour commission devant qui tout se passera le P. Goüye, M ${ }^{\text {rs }}$. Cassini et de la Hire ». Voir Procès-verbaux de l'Académie royale des sciences, 1705, séance du 8 août, f. $263 \mathrm{v}-$ $264 \mathrm{r}$ : « sur la contestation qui est depuis long tems dans les Journaux entre $\mathrm{M}^{\mathrm{rs}}$. Rolle et Saurin sur la Methode des Infiniment petits, $\mathrm{M}^{\mathrm{r}}$. Saurin ayant demandé à $\mathrm{M}^{\mathrm{r}}$. l'Abbé Bignon des Commissaires de l'Académie, il a nommé le P. Goüye, $\mathrm{M}^{\mathrm{rs}}$. Cassini, de la Hire, Gallois, et moy [Fontenelle] ».

${ }^{219}$ L'Hospital à Huygens le 4 mars 1695, dans HuYGENS, Correspondance 1691-1695, 1905, p. 711-712.

${ }^{220}$ Leibniz à l'Hospital le 18 mars 1695, dans LEIBNIZ, Sämtliche Schriften und Briefe, 2004, p. 317-318. Voir aussi 13/23 mai 1695, p. 365 : « je trouve fort bon que Monsieur de la Hire demonstre les nouvelles découvertes à la façon des anciens Geometres et on luy en aura de l'obligation, parce qu'il rend ainsi temoignage de la verité. Mais il aura souvent besoin de beaucoup de paroles ».

${ }^{221}$ Leibniz à l'Hospital le 4/14 décembre 1696, dans LEIBNIZ, Sämtliche Schriften und Briefe, 2011, p. 216.

222 Varignon à J. I Bernoulli le 6 août 1697, dans BERNOULLI, Der Briefwechsel mit Pierre Varignon, 1988, p. 124. 
faut compter La Hire parmi ces mathématiciens ${ }^{223}$. La Hire est aussi décrit comme un juge n'osant se prononcer : selon Varignon l'abbé Jean Gallois, académicien, se montre favorable aux critiques de Rolle et «M. Cassini, M. de la Hire, et le P. Gouye Jesuite, n’ont osé se prononcer, de peur de condanner l'Abbé Galoys avec M. Rolle 224 »; l'Abbé Galloys est dit «ennemi déclaré $[\ldots]$ du calcul » et La Hire est « livré » à Galloys ${ }^{225}$. Concernant les débats opposants Rolle et Saurin et la nomination d'une commission Leibniz juge que «M. l'Abbé Gallois y est jamais entré assez [dans le calcul] pour en juger : mais M. de la Hire ne voit que trop la bonté de ce calcul, et il en est jaloux autant que je puis le conjecturer ${ }^{226} »$. Jacques Lelong écrit à Leibniz au sujet du jugement de cette commission que " $\mathrm{M}^{\mathrm{r}}$ de la Hire qui a honte de devenir disciple apres avoir eté maitre a agi secretement, n'ayant pas osé se declarer publiquement ${ }^{227} »$. Ainsi, La Hire qui critique le calcul dans ses fondements ferait partie des mathématiciens du «vieux stile». Bien que membre de commissions, il n'oserait pas se prononcer, et comprenant le calcul il s'en montrerait jaloux, ne voulant pas devenir « disciple » notamment d'académiciens plus jeunes.

C'est dans ce contexte qu'entre le $1^{\text {er }}$ et le 15 juin 1697 Varignon et La Hire lisent à l'Académie des mémoires concernant la chute libre sur la cycloïde en démontrant en particulier par la géométrie sa propriété d'isochronisme ${ }^{228}$. Le 4 janvier 1698 Varignon détermine avec le calcul différentiel des courbes isochrones pour tous types d'hypothèses d'accélération ${ }^{229}$. Le 5 juillet et le 6 septembre Varignon formule des « règles générales » de vitesses instantanées en s'appuyant sur l'algorithme leibnizien pour des trajectoires rectilignes et curvilignes, et les applique notamment à la détermination des expressions des temps de chute libre d'un mobile sur un cercle et une cycloïde ${ }^{230}$. Dans les séances académiques des 9 et 30 août, La Hire trouve aussi ces temps pour les mêmes courbes à l'aide, lui aussi, d'une « regle generale ${ }^{231}$. Nous examinons l'élaboration et l'application de cette "règle" et donnons quelques éléments de comparaison de ces travaux avec ceux de Varignon $^{232}$. Nous remarquons, en particulier, que les techniques mises en œuvre par La Hire, qui ressortissent à la géométrie infinitésimale, sont tributaires de la possibilité d'interpréter géométriquement des propositions de la cinématique galiléenne en fonction de la trajectoire étudiée ; ces recherches n'atteignent alors pas la généralité de celles de Varignon qui utilise des expressions analytiques quelles que soient les courbes et les accélérations.

Le début du mémoire de La Hire est purement géométrique. Entre les parallèles $B M$ et $D N$ (Fig. 17) il trace un demi-cercle $B O D$ de centre $C$ et de rayon $C B$. La corde $B O$ prolongée coupe $D N$ en $E$, et $F R$ passant par $O$ et parallèle à $D N$ est telle que par construction $F R=B E$. Avec $B F=y, B D=r$, les propriétés du cercle donnent $B O^{2}=B F . B D$ soit $B O=\sqrt{r y}$. Par ailleurs, $B O D$ et $B D E$ sont des triangles semblables, et donc $\frac{B F}{B O}=\frac{B D}{B E=F R}$ soit $F R=\frac{r^{2}}{\sqrt{r y}}$ ou $x=\frac{r^{2}}{\sqrt{r y}}$ avec $F R=x^{233}$.

\footnotetext{
${ }^{223}$ J. I Bernoulli à Leibniz le 14 août 1697, dans LEIBNIZ, Sämtliche Schriften und Briefe, 2011, p. 560-561.

${ }^{224}$ Varignon à Leibniz le 10 mai 1705, dansibid., p. 420 (à paraitre, en ligne voir https://leibnizedition.de/reihen/reiheiii/ consulté le 12/02/2019).

${ }^{225}$ Varignon à Leibniz le 9 octobre 1705, ibid., p. 473 (à paraittre, voir https://leibnizedition.de/reihen/reihe-iii/ consulté le 12/02/2019).

${ }^{226}$ Leibniz à Varignon le 6 novembre 1705, ibid., p. 502 (à paraittre, voir https://leibnizedition.de/reihen/reihe-iii/ consulté le 12/02/2019).

${ }^{227}$ Lelong à Leibniz le 8 mars 1706, dans ibid., 2017, p. 702-703.

228 Voir VARIGNON, « Nouvelle Demonstration des mouvemens Isochrones », 1697, Varignon, « Seconde et Troisiéme Demonstration des mouvemens Isochrones », 1697, VARIGNON, "Nouvelle Remarque sur les mouvemens Isochrones », 1697. LA HIRE, «Démonstration du temps qu'un corps employe a tomber », 1697.

${ }^{229}$ VARIGNON, « Manière generale de trouver les courbes Isochrones », 1698.

${ }^{230}$ VARIGNON, «Regle generale pour toutes sortes de mouvement », 1698 et VARIGNON, «Application de la Regle genrale », 1698.

${ }^{231}$ LA HIRE, « Regle generale », 1698.

232 Sur les règles de Varignon et leurs applications, voir BLAY, La naissance de la mécanique analytique, 1992, p. 153-179.

${ }^{233}$ LA HIRE, « Regle generale », 1698, f. 368r-368v. Les points $R$ forment une « une hyperbole du second genre ».
} 


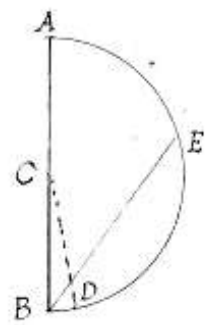

Fig. 16 : La Hire, « Remarque sur l’usage », 1697, f. 24v

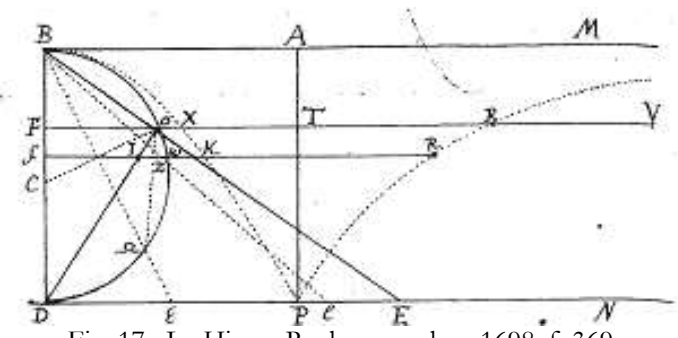

Fig. 17 : La Hire, « Regle generale », 1698, f. 369v

La suite est de nature cinématique. La Hire considère que «les ligne $F R$ ordonnées [...] representent les temps de la chutte d'un corps par un même espace indéfiniment petit comme $F f$ à la hauteur de ces ordonnées » (Fig. 17). La Hire divise ici $B D$ en grandeurs $F f$ égales, et tire à chaque $F$ les ordonnées $F R$. Il interprète ces $F f$ comme des hauteurs de chute libre et montre alors que «si les espaces sont verticaux comme $F f$ et qu'ils soient compris entre chaque parallele, les rectangles entre ces paralleles representent les temps de la chutte du corps par ces espaces ». En effet, d'après la cinématique galiléenne, « toutes les cordes comme $B O, B \omega, B \Omega, B D$, dans le demicercle representent les vitesses d'un corps descendu depuis le repos en $B A$ jusqu'aux hauteurs $O, \Omega, D »^{234}$. Puis si « un meme espace indefiniment petit [est] parcouru uniformement » avec ces vitesses, alors ces dernières sont en proportion inverse des temps. Or, La Hire montre que $F R \propto$ $\frac{1}{B O}{ }^{235}$, autrement dit qu'on a une relation du type BO.FR.Ff $=B \omega . f R . F f:$ avec $B O$ et $B \omega$ des vitesses uniformes lors des parcours de deux espaces égaux à $F f, F R . F f$ et $f R . F f$ représentent les durées des parcours. Par ailleurs, si le corps ne chute pas verticalement mais suivant un plan incliné comme $O I$ ou $O K$, «si l'on fait un rectangle $F R \times O I$ ou $O K$ et qu'on l'applique à $F f$, on aura la ligne $F V$ dont le rectangle $F V \times F f$ represente le temps de la chutte du corps par $O I$ ou $O K »$. En effet, si l'ordonnée $F V$ est telle que $F V \times F f$ mesure la durée de chute sur $O I$, puisque $F R \times F f$ mesure celle sur $F f$ et que d'après une proposition de Galilée les durées des chutes d'un corps sur des plans diversement inclinés de même hauteur sont comme la longueur de ces plans ${ }^{236}$, ainsi $\frac{F V \times F f}{F R \times F f}=\frac{O I}{F f}: F V \times F f=F R \times O I$ est la « regle generale », et $F V$ sont les temps pour une chute sur une courbe dont $O I$ est un élément infinitésimal ${ }^{237}$.

La Hire considère la chute sur un arc cercle $G Q D$ (Fig. 18) de rayon $B G=B D=B Q=r$. Le corps part de $B G M$, et si on trace la courbe $P R R$ par la méthode décrite ci-dessus, $F R \times F f$ représente la durée de chute sur l'espace $F f$, tandis que $F R \times F f \times Q q$ représente la durée de la chute le long de l'arc $Q q ; F R \times Q q$ donne l'ordonnée $F V$ qui permet de tracer la courbe des temps du parcours du corps sur le cercle. En effet, la cinématique galiléenne donne $t_{Q q}$ le temps de chute le long de l'arc $Q q$, avec $t_{Q q}=F V \times F f=\frac{Q q}{F f}$. $F R . F f^{238}$, et donc $F V \times F f=F R \times Q q$; «le rectangle $F V \times F f$ representera le [...] temps de la chutte du corps par l'arc $Q q$, comme le rectangle $F f \times F R$ represente le temps de la chutte du corps par $F f$ ». Il est à noter que manifestement La

\footnotetext{
${ }^{234}$ La Hire ne précise pas la raison de ceci. Les temps de descente le long des cordes sont égaux, voir GALILEI, Discorsi e dimostrazioni matematiche, 1898, p. 221-223. Par ailleurs, Galilée montre qu'un corps en chute libre parcourt pendant une certaine durée une distance deux fois plus petite que celle qu'il franchit pendant la même durée avec pour vitesse uniforme celle acquise à la fin de la chute libre (ibid., p. 208-209). Ainsi, avec $v$ et $v^{\prime}$ deux vitesses acquises au bout de $\Delta t, 2 B O=v \cdot \Delta t$ et $2 B \omega=v^{\prime} . \Delta t$, autrement dit, les cordes « representent » selon le mot de La Hire les vitesses.

${ }^{235} \mathrm{Car} B O \Omega$ et $B \varepsilon E$ (Fig. 17) sont semblables (car $\widehat{B D O}=\widehat{B O \Omega}$ et $\widehat{B D O}=\widehat{B E D}$ ) et ainsi $\frac{B E=B F}{B \varepsilon}=\frac{B \Omega}{B O}$.

236 GALILEI, Discorsi e dimostrazioni matematiche, 1898, p. 215-219.

${ }^{237}$ LA HiRe, «Regle generale », 1698, f. 369v-370v. La Hire n’écrit pas "infinitésimaux", OI comme Ff sont dits « indéfiniment petits ». Voir f. $374 \mathrm{v}$ pour l'appellation « regle generale ».

${ }^{238}$ Le rapport des temps de chute $t_{Q q}$ et $F R . F f$ est comme celui des longueurs $Q q$ et $F f$.
} 
Hire assimile ici l'arc $Q q$ à la corde $Q q$, et ce malgré ses réticences à ce sujet ${ }^{239}$. La Hire va alors chercher une expression de l'arc $Q q$ dont le produit par $F R$ et le quotient par $F f$ conduit à $F V$. Si on nomme $Z$ l'intersection de la normale tirée de $q$ sur $F R, q Z Q$ et $B Q F$ sont semblables soit $\frac{F Q}{B Q}=$ $\frac{Z q}{Q q}$. Avec $Z q=F f=i$, et puisque $F Q=\sqrt{B Q^{2}-B F^{2}}=\sqrt{r^{2}-y^{2}}$, il vient $Q q=\frac{r i}{\sqrt{r^{2}-y^{2}}}$. Or, la durée de chute le long de $Q q$ vaut $F R \times Q q=F V \times F f$, et comme $F R=\frac{r^{2}}{\sqrt{r y}}$ on obtient $F V \times$ $F f=\frac{r^{3} i}{\sqrt{r^{3} y-r y^{3}}}(1)$, soit $F V$ avec $F f=i$.

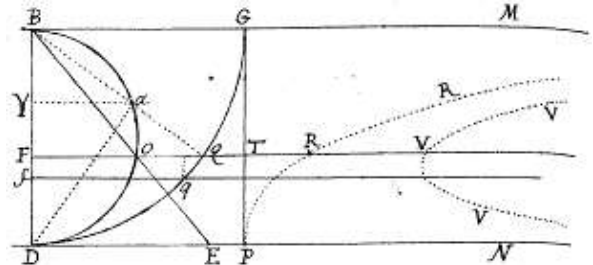

Fig. 18 : LA HIRE, « Regle generale», 1698, f. 372r

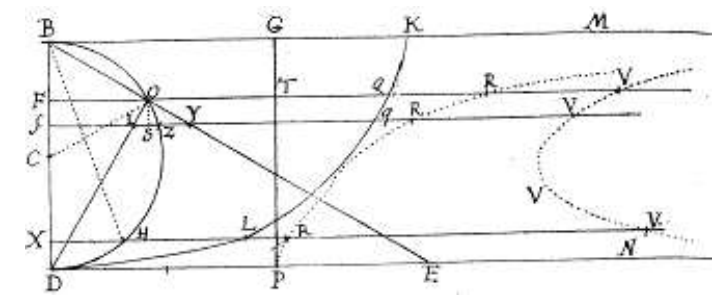

Fig. 19 : LA HiRE, « Regle generale », 1698, f. 374v

La même technique est à l'œuvre pour une cycloïde $K Q D$ (Fig. 19) de cercle générateur $B O D$. La Hire calcule $Q q=O I$ ce qui revient à assimiler l'élément de courbe à sa tangente ${ }^{240}$. Avec $S$ la projection orthogonale de $O$ sur $f r, \frac{F O}{B O}=\frac{F f=O}{O I=Q q}$ (car $B O F$ et $O S I$ semblables) et puisque $B O=$ $\sqrt{r y}$ et $B F=y$, il vient $F O=\sqrt{r y-y^{2}}$ ce qui donne $Q q$; avec $F f=i$ et $F R=\frac{r^{2}}{\sqrt{r y}}$, on aura par la « regle generale », suivant la terminologie de La Hire, $F V \times F f=F R \times Q q=\frac{r^{2} i}{\sqrt{r y-y^{2}}}(2)$ soit $F V$.

La relation (1) peut s'interpréter comme $d t=\frac{r^{3} d y}{\sqrt{r^{3} y-r y^{3}}}$ et (2) $d t=\frac{r^{2} d y}{\sqrt{r y-y^{2}}}$, avec $i=d y$, qui sont les expressions que Varignon obtient dans son mémoire du 6 septembre 1698 en appliquant au mouvement curviligne ce qu'il nomme une « Regle generale » qu'il énonce et applique le 5 juillet 1698 pour les mouvements rectilignes. Cette règle consiste en des expressions analytiques de la vitesse instantanée, des temps et des espaces. Ainsi, avec $A B=x$ (Fig. 20) l'espace parcouru lors d'une trajectoire rectiligne, avec $B C=y$ les vitesses à chaque point $B$ données par la courbe $C C$, et avec $B E=z$ sur la courbe $E E$ le temps employé pour aller de $A$ et $B$, on aura a $d z=\frac{d x}{y}$. Si le corps se meut sur la courbe $G G$, avec $B G=v$ à l'instant $B H=s$ donné par $H H$, on a $d s=$ $\frac{\sqrt{d x^{2}+d v^{2}}}{y}$ avec $y$ la vitesse instantanée de parcours de $\sqrt{d x^{2}+d v^{2}}$ pendant $d s$. L'obtention d'une relation du type $d z=\frac{d x}{y}$ repose sur le fait que pendant $d t$ la vitesse instantanée peut être considérée comme uniforme car $y \pm d y=y$ d'après la première "Demande » de l'Analyse des infiniment petits de L'Hospital ${ }^{241}$. Il suffit alors à Varignon de déterminer une expression de $d v$ à partir de la courbe donnée (un cercle ou une cycloïde) et, en connaissant la loi d'évolution des vitesses - par exemple, pour une chute libre, $y=\sqrt{a x}$ avec $a$ une constante - de remplacer $y$ et $d v$ par les expressions obtenues dans $d s=\frac{\sqrt{d x^{2}+d v^{2}}}{y}$ pour obtenir la courbe des temps ${ }^{242}$.

239 Voir LA HiRE, « Remarque sur l'usage », 1697 examiné ci-dessus.

${ }^{240} O I$ est normale à $B O$ en $O$ et, par les propriétés de la cycloïde, $O D$ est parallèle à la tangente en $Q$ de $K Q D$.

${ }^{241}$ L'Hospital, Analyse des infiniment petits, 1696, p. 2-3.

242 Pour tout ceci, VARIGNON, « Regle generale pour toutes sortes de mouvement », 1698 et VARIGNON, « Application de la Regle genrale », 1698. Pour une analyse détaillée voir BLAY, La naissance de la mécanique analytique, 1992, p. 153-171. 


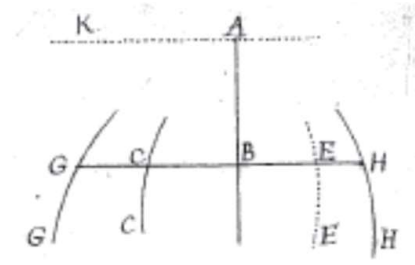

Fig. 20 : VARIGNON, « Application de la Regle », 1698, f. 387v

En traçant le cercle $B O D$ et les ordonnées $F R$ (Fig. 17), La Hire se donne d'abord des configurations géométriques qu'il interprète ensuite cinématiquement. Ainsi, la construction conduit à $F R \propto \frac{1}{B O}$ et puisque $B O$ est une corde, une proposition de Galilée montre qu'elle peut alors représenter une vitesse et, par conséquent, les règles (galiléennes) des mouvements uniformes conduisent à voir en $F R \times F f$ ou la surface $F f r R$ comme la durée de parcours de $F f$ avec une vitesse uniforme $B O$. Ceci acquis, c'est à nouveau une proposition de cinématique galiléenne sur la chute sur des plans inclinés qui permet dénoncer la "regle generale" $F V \times F f=F R \times Q q$ que La Hire peut appliquer systématiquement à différentes courbes. En suivant les notations de Varignon ci-dessus, $F V \times F f=F R \times Q q$ peut s'interpréter comme $d z=\frac{d x}{y}$ avec $F R$ l'inverse d'une vitesse instantanée ; les deux savants appliquent une règle semblable à différentes courbes. Malgré tout, ils se séparent sur les fondements d'une telle règle qui repose chez La Hire sur une géométrie infinitésimale et non sur une interprétation cinématique de l'algorithme leibnizien qui par la relation $y \pm d y=y$ permet de considérer $y$ comme uniforme et d'appliquer alors les règles galiléennes de ces mouvements. Sans doute La Hire est-il fidèle ici à ses critiques de février 1697 à l'encontre du calcul différentiel bien qu'il assimile ci-dessus des arcs élémentaires de cercle à des cordes. Quoi qu'il en soit, une fois ses relations géométriques trouvées, La Hire ne cherche pas à les transcrire analytiquement et à utiliser l'algorithme du calcul différentiel ${ }^{243}$, pas plus qu'il ne fonde des concepts - celui de la vitesse instantanée - analytiquement. Par ailleurs, sa technique reste tributaire de la chute libre et dans ce cadre aux propositions galiléennes concernant l'identification dans un cercle d'une vitesse instantanée à une corde ou concernant les plans inclinés. La Hire ne donne pas d'autres lois d'évolution des vitesses, lois qui nécessiteraient des constructions complexes au cas par cas pour les ordonnées $F R$, tandis qu'à partir de janvier 1700 Varignon énonce des règles analytiques quelles que soient les forces dites "accélératrices ${ }^{244}$. Bien que La Hire formule une "regle generale", celle-ci est subordonnée à la géométrie et à des figures adéquates à une interprétation cinématique - le cercle est tracé pour donner une corde qui représente une vitesse dans le seul cadre de la science galiléenne.

Dans ce manuscrit de 1698, La Hire propose aussi une solution géométrique pour la courbe brachystochrone ou, plus précisément, il montre que l'arc de la cycloïde vérifie la propriété du chemin le plus court possible. $Q q$ est « un arc indefiniment petit » de cyclö̈de de cercle générateur $B O D$ que le cercle de centre $q$ et de rayon $Q q$ coupe en 8 (Fig. 21 et 22). Tout d'abord, du fait des propriétés de la cycloïde, puisque $O I$ et $Z 8$ sont sur des cordes du cercle générateur (Fig. 22) lesquelles sont parallèles aux tangentes à la cyclö̈de en $Q$ et $q$, on a $Z 8=q 8$ et $O I=Q q$. Les triangles semblables $Q q p$ ( $q p$ normale à $F R$ en $p$ ) et $B D O$ (Fig. 22) donnent $\frac{O p}{O I}=\frac{B O}{B D}(1)$; les triangles semblables $Z 8 t^{245}$ et $B D Z$ donnent $\frac{Z 8}{Z t}=\frac{B D}{B Z}$ (2). En combinant (1) et (2), avec $Z 8=q 8=$

\footnotetext{
243 Telle est la pratique de travaux autour de 1690 portant sur la recherche de courbes aux propriétés cinématiques particulières ; le problème physique est ramené à un problème géométrique dont la résolution peut donner lieu au remplacement des grandeurs infinitésimales par l'algorithme du calcul différentiel et intégral. Voir BLAY, La naissance de la mécanique analytique, 1992.

244 Voir BLAY, La naissance de la mécanique analytique, 1992, p. 180-221.

${ }^{245} T 8$ est normale $f R$ en $T$ (Fig. 21) et correspond à $t 8$ sur la Fig. 22.
} 
$O I$ et $O p=Q p$, il vient $\frac{Q p}{Z t}=\frac{B O}{B Z}$. D'après les démonstrations précédentes, les cordes $B O$ et $B Z$ sont les vitesses en $O$ et $Z$, lesquelles sont « comme les raretés du milieu au dessous de $F R$ et de $f R »^{246}$, donc « un corps pesant qui ira du point $Q$ au point 8 en passant par le point $q$ ou $I$ et au travers des milieux qui sont dans la raison $\frac{I S}{I T}$ ou de $Q p$ à $Z t$ y passera dans le moins de temps $»^{247}$. Autrement dit, la courbe est telle qu'elle vérifie $\frac{\sin i}{v}=K$, avec $i$ l'angle entre la courbe décrite par le corps et une verticale, $v$ la vitesse du corps et $K$ une constante. La Hire recourt ici implicitement à une analogie avec l'optique et s'appuie sur le principe de Fermat qui stipule qu'un rayon passant d'un milieu plus rare à un moins rare est réfracté de telle sorte que le temps de parcours est le moindre possible, principe à partir duquel Fermat déduit la loi des sinus. Or, ici, le rapport $\frac{Q p}{Z t}$ représentant "la raison" des "milieux", autrement dit, étant lié à leur "rareté", est aussi celui des sinus d'incidence et de réfraction ; la vitesse du corps pesant sur la courbe est celle acquise lorsqu'il tombe en chute libre verticalement des hauteurs $B F, B f$ etc., et ce sera celle à chaque instant dans le milieu dont la « rareté » varie.

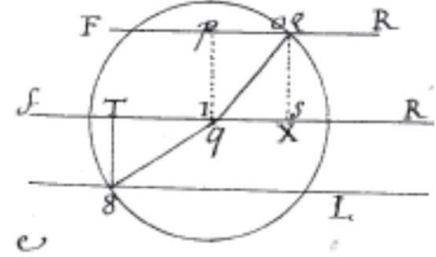

Fig. 21 : LA HIRE, « Regle generale », 1698, f. 379r

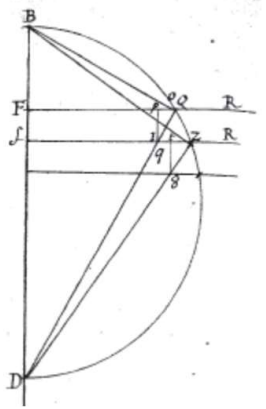

Fig. 22 : LA HIRE, « Regle generale », 1698, f. 79v

La Hire conclut que «ce Problême a esté proposé et resolu par M. ${ }^{\mathrm{r}}$ Bernoulli professeur a Groningue et par plusieurs autres Mathematiciens ${ }^{248} »$. De fait, La Hire, comme Jean Bernoulli, s'appuie sur le principe de Fermat, mais Bernoulli donne l'analyse du problème auquel il applique le calcul différentiel et intégral, tandis qu'ici La Hire vérifie que la cycloïde correspond bien à la condition de plus rapide descente ${ }^{249}$. En 1702, La Hire évoque à nouveau la brachystochrone dans ses recherches sur la trajectoire d'un rayon lumineux dans l'atmosphère. Il détermine la nature de la courbe qui répond à la condition de plus rapide descente en s'appuyant cette fois-ci explicitement sur le principe de Fermat, mais toujours sans recourir au calcul différentiel ${ }^{250}$. Cette publication et

246 Plus le milieu est dit «rare» plus la vitesse d'un corps qui s'y meut y est élevée, ces deux grandeurs étant proportionnelles. On retrouve ceci dans La Hire, «Examen de la ligne courbe », 1704, p. 57-58.

${ }^{247}$ LA HIRE, « Regle generale », 1698, f. 379r-379v.

${ }^{248}$ Ibid., f. 379v. Notamment Jacques et Jean Bernoulli, Leibniz et L'Hospital qui donnent des solutions du problème, voir BLAY, La naissance de la mécanique analytique, 1992, p. 77-98.

${ }^{249}$ Pour la solution de Bernoulli, voir BLAY, La naissance de la mécanique analytique, 1992, p. 87-90.

${ }^{250}$ Voir LA HiRE, «Sur la ligne Courbe », 1702, p. 57-59 publié dans les mémoires de l'académie, LA HiRE, « Examen de la ligne courbe », 1704. En prenant $D$ (Fig. 22) sur la surface de la Terre, La Hire considère que les « extensions des ressorts de l'air » de l'air en $F, f$ etc. sont représentées par leurs quantités proportionnelles à $D O, D Z$ etc. qui sont comme $\sqrt{D F}, \sqrt{D f}$ etc. : autrement dit, vers la surface l'atmosphère et ces « ressorts » sont davantage comprimés, l'air y est plus dense et s'oppose alors plus au passage du rayon lumineux. La Hire remarque que si les rayons lumineux ont des directions $Q p, q 8$ etc. (Fig. 21), soit encore $Q q$ et $Z 8$ (Fig. 22) alors « ces parties seront des Elemens de Cycloïde » $(Q q$ et $Z 8$ sont des éléments des tangentes à la courbe et sont le long de cordes du cercle générateur). Il montre alors dans ce cas, par de simples considérations de triangles semblable, que les sinus des angles d'incidences en $q$ et 8 sont proportionnels à $D O, D Z$ etc. soit à $\sqrt{D F}, \sqrt{D f}$ etc. Notons que $D O, D Z$ peuvent s'interpréter comme les vitesses d'un corps s'élevant verticalement de $D$ vers $B$ selon la loi de chute libre et, finalement, les techniques à l'œuvre dans ce mémoire ne diffèrent pas de celles du manuscrit de 1698. 
le manuscrit de 1698 sont ses derniers travaux sur de tels sujets et, finalement, tout se passe comme si sa participation aux débats de l'époque et aux défis que se lancent les promoteurs du calcul différentiel et intégral prenait la forme d'un pas de côté par rapport à la nouveauté.

\section{Conclusion}

Dans un premier temps, cet article présente le cadre philosophique dans lequel la physique de Philippe de La Hire s'inscrit, puis il propose une étude de la mécanique du savant en s'attachant, en particulier, à des questions liées à son axiomatique, à ses principes, à ses méthodes, aux développements techniques qu'elle propose, ou encore à ses rapports à certaines évolutions qui marqueront durablement le $18^{\mathrm{e}}$ siècle.

La faible quantité de travaux liés à la philosophie mécanique suggère que La Hire n'est pas un auteur à systèmes. Malgré tout, sa physique est corpusculaire et il puise des principes et des méthodes explicatives aussi bien chez Descartes, Gassendi et des chimistes de la fin du $17^{\mathrm{e}}$ siècle, une multiplicité de sources qui témoigne en retour de la diversité des formes prises par le mécanisme qui ne saurait être réduit à une doctrine. La Hire fait partie de ce que certains nomme les "Cartésiens" et son explication du froid et du gel subit le sort réservé aux "hypothèses" ou aux "systèmes" au cours du $18^{\mathrm{e}}$ siècle : dans l'Encyclopédie, De Ratte rejette ce genre d'explications jugées non fondées sur l'observation et l'expérimentation ${ }^{251}$.

Comme l'indique leurs sous-titres, le traité sur les épicycloïdes et celui de mécanique consacrent une part importante à «l'usage dans les méchaniques » de ces courbes et à expliquer «ce qui est necessaire dans la pratique des Arts ». La contribution de La Hire prend avant tout la forme d'une application de la géométrie aux arts, plutôt que l'élaboration et l'usage de lois expérimentales de frottements, que la conceptualisation du travail mécanique et que l'optimisation de l'effort d'une machine, sujets au cœur de travaux d'Amontons et de Parent et plus globalement de recherches tout au long du $18^{\mathrm{e}}$ siècle ${ }^{252}$. Par ailleurs, les éléments de statique qui constituent le fondement de l'étude des machines reçoivent dans leur exposition un soin particulier qui se manifeste par un attachement aux "Anciens" à travers l'axiomatique et les procédures de démonstrations mises en œuvre. La Hire critique alors des "Modernes" parmi lesquels il faut probablement inclure certains de ses confères académiciens comme Varignon.

La dynamique du Traité de mécanique, tant du point de vue conceptuel, en particulier concernant la force, que des procédures mathématiques qu'elle mobilise, fait écho à des travaux de la deuxième moitié du $17^{\mathrm{e}}$ siècle, notamment ceux de Mariotte, de Huygens ou encore ceux de Varignon du début des années 1690. En dynamique comme en cinématique, La Hire s'appuie sur la géométrie infinitésimale et ses écrits ressortissent à une période pré-analytique, antérieure à l'élaboration par Varignon des concepts de vitesse et d'accélération instantanées à l'aide de l'algorithme leibnizien du calcul différentiel et intégral. On écrit alors que La Hire privilégie la synthèse à l'analyse et on le qualifie de "vieux style". La Hire préconise de s'assurer des résultats obtenus par le calcul différentiel à l'aide de « la géométrie ordinaire » qui serait donc plus certaine ${ }^{253}$. Selon Fontenelle, « ce n'est pas qu'il [La Hire] ne sçût l'Analise moderne [...] mais il avoit pris de jeune l'autre pli ${ }^{254} »$. Un rapprochement mérite sans doute d'être fait entre La Hire et Huygens au sujet duquel l'historien H. J. M. Bos souligne la «manière géométrique de penser». Ainsi, concernant le problème de la courbe dite isochrone proposée par Leibniz, Huygens s'en tient à la géométrie infinitésimale et à des proportions pour ensuite transformer en toute fin le problème résolu en termes algébriques. Mais des manuscrits montrent sa résolution en utilisant le calcul leibnizien. Manifestement, ce dernier ne lui semble pas meilleur que sa solution géométrique et l'historien souligne alors que le

\footnotetext{
251 Voir en particulier RATTE, «Froid », Encyclopédie, 1757, t. VII, p. 313a. Sur la critique des systèmes et en particulier ceux sur le froid dans l'Encyclopédie, voir CHABOT, BRETEUIL, « La réception épistémologique », 2015.

${ }^{252}$ Sur le travail et l'optimisation au 18 siècle, voir FONTENEAU, Développements précoces du concept de travail, 2011.

${ }^{253}$ LA HIRE, « Remarque sur l'usage », 1697, f. 27r.

${ }^{254}$ FONTENELLE, «Eloge de M. de La Hire », 1719, p. 88.
} 
travail de Huygens est le "point culminant » de ce style et, en même temps « sa limite » du fait du manque de généralité et de la complexité de la méthode ${ }^{255}$. Des conclusions manifestement applicables aux travaux de La Hire.

255 BOS, «L'élaboration du calcul infinitésimal », 1982, p. 115-122. 


\section{Bibliographie citée}

Abréviations :

HARS 1733 : Histoire de l'Académie royale des sciences. Année M.DCCXXXIII. Avec les Mémoires de Mathématiques \& de Physique pour la même Année. Tirés des Registres de cette Académie, Paris, Imprimerie royale, 1735. Partie " Histoire ».

MARS 1733 : Histoire de l'Académie royale des sciences. Année M.DCCXXXIII. Avec les Mémoires de Mathématiques \& de Physique pour la même Année. Tirés des Registres de cette Académie, Paris, Imprimerie royale, 1735. Partie « Mémoires ».

AmOntOns G., «De la resistance causée dans les Machines tant par les frotemens des parties qui les composent que par la roideur des cordes qu'on y emploie et la manière de calculer l'un et l'autre ", Procès-Verbaux de l'Académie royale des sciences, t. XVIII, 12 novembre 1698-23 décembre 1699, séance du 19 décembre 1699, f. 573r-583v.

Amontons G., «De la resistance causée dans les machines, tant par les frottemens des parties qui les composent, que par la roideur des cordes qu'on y employe, \& la maniere de calculer l'un et l'autre ", MARS 1699, 1701, p. 206227.

ANSTEY P. R., «Le ressort de l'air selon Boyle et Mariotte », La philosophie naturelle de Robert Boyle, DENNEHY M., RAMOND C. (éds.), Paris : Vrin, 2009, p. 379-403.

ARChImede, De l'équilibre des plans et de leurs centres de gravité, dans CEuvres d'Archimède, PEYRARD F. (éd.), Paris: F. Buisson, 1807, p. 275-317.

BARTHEZ P. J., "Force des animaux », Encyclopédie ou Dictionnaire raisonné des Sciences, des Arts et des Métiers, par une Societé de Gens de Lettres, Diderot D., D’Alembert J. Le Rond (éds.), vol. VII, Paris : Briasson, David l'aîné, Le Breton, Durand, 1757, p. 120b-124b.

BEAUVAL H. B. de, Dictionnaire Universel contenant generalement tous les mots françois tant vieux que modernes, \& les termes de toutes les Sciences \& les Arts, 3 vol., La Haye : Rotterdam, A. et R Leers, 1701.

BEAUval H. B. de, Dictionnaire Universel contenant generalement tous les mots françois tant vieux que modernes, \& les termes de toutes les Sciences \& les Arts, 3 vol., Rotterdam R. Leers, 1708.

BECCHI A., «Idées manuscrites, théories imprimées : la mécanique architecturale de Philippe de La Hire », Philippe de La Hire 1640-1718. Entre architecture et sciences, BECCHI A., RousteAu-CHAMBON H., SAKAROVITCH J. (éds.), Paris : A. et J. Picard, 2013, p. 177-190.

Bernier F., Abregé de la philosophie de Gassendi, 8 t., Lyon Anisson et Possuel, 1678.

Bernoulli J. (I), Der Briefwechsel mit Pierre Varignon, Costabel P., Peiffer J. (éds.),Bâle : Birkhäuser Verlag, 1988.

Bertoloni Meli D., « Guidobaldo dal Monte and the Archimedean Revival », Nuncius, 7/1, 1992, p. 3-34.

BLAY M., «Deux moments de la critique du calcul infinitésimal : Michel Rolle et George Berkeley », Revue d'Histoire des Sciences, 39/3, 1986, p. 223-253.

BLAY M., La naissance de la mécanique analytique. La science du mouvement au tournant des XVII et XVIII siècles, Paris : PUF, 1992,

Bos H. J. M., « L'élaboration du calcul infinitésimal, Huygens entre Pascal et Leibniz », Huygens et la France, Paris : Vrin, 1982, p. 115-122.

BOYLE R., New Experiments Physico-Mechanical, touching the Spring of the Air, and its Effects, dans The works of Robert Boyle, vol. I, HunTer M., DAvis E. B. (éds.), London : Pickering \& Chatto, 1999-2000.

BOYLE R., Some Specimens of an Attempt to make Chymical Experiments useful to illustrate the notions of the Corpuscular Philosophy, dans The works of Robert Boyle, vol. II, HunTER M., DAvis E. B. (éds.), London : Pickering \& Chatto, 1999-2000.

Camus Ch. E. L., «Des Dents des Roues, et des Ailes des Pignons, pour rendre les Horloges plus parfaites ", MARS 1733, 1735, p. 117-140.

Снавот H., Breteil N., "La réception épistémologique de l'œuvre scientifique de Dortous de Mairan dans l'Encyclopédie », Revue d'Histoire des Sciences, 68/2, 2015, p. 375-404.

Clavelin M., La philosophie naturelle de Galilée, Paris : A. Michel, 1968.

Clericuzio A., "A redefinition of Boyle’s chemistry and corpuscular philosophy », Annals of Science, 47, 1990, p. 561 589.

Clericuzio A., Principles and Corpuscules. A Study of Atomism and Chemistry in the Seventeenth Century, Dordrecht : Kluwer Academic Publishers, 2000.

D’Alembert J. Le Rond, «Ether », Encyclopédie ou Dictionnaire raisonné des Sciences, des Arts et des Métiers, par une Societé de Gens de Lettres, VI, DiDerot D., D’Alembert J. Le Rond (éds.), Paris : Briasson, David, Le Breton, Durand, 1756, p. $51 \mathrm{a}-51 \mathrm{~b}$.

D’Alembert J. Le Rond, «Physique », Encyclopédie ou Dictionnaire raisonné des Sciences, des Arts et des Métiers, par une Societé de Gens de Lettres, XII, Diderot D., D’Alembert J. Le Rond (éds.), Neufchastel : Faulche et Compagnie, 1765, p. 539a-540a.

DE GANDT F., «L'analyse de la percussion chez Galilée et Torricelli », L'cuvre de Torricelli : science galiléenne et nouvelle géométrie, DE GANDT F. (éd.), Nice : Faculté des Lettres et Sciences Humaines, 1989, p. 57-77.

De GandT F., Force and Geometry in Newton's Physics, Princeton : Princeton University Press, 1995.

Desaguliers J. T., A Course of Experimental Philosophy, vol. I, seconde édition, London : Innys \& al., 1745.

Desaguliers J. T., Cours de physique expérimentale, t. I, Paris : J. Rollin, C.-A. Jombert, 1751. 
Descartes R., Les météores, dans Euvres de Descartes, VI, AdAM C., TANNERY P. (éds.), Paris : Vrin, 1996.

Descartes R., Principes de la philosophie, dans CEuvres de Descartes, IX, ADAM C., TANnery P. (éds.), Paris : Vrin, 1996.

Descartes R., Euvres de Descartes, 11 vol., AdAM C., TANnery P. (éds.), Paris : Vrin, 1996.

Dictionnaire Universel François et Latin, 1721, 3 vol., Trévoux : Ganeau.

Dictionnaire Universel François et Latin, 1721, 5 vol., Trévoux : Delaulme.

Diderot D., D’Alembert J. Le Rond (éds.), Encyclopédie ou Dictionnaire raisonné des Sciences, des Arts et des Métiers, par une Societé de Gens de Lettres, 1751-1772, Paris: Briasson, David, Le Breton, Durand, puis Neufchastel : Faulche et Compagnie, $28 \mathrm{t}$.

FONTENELLE B. Le Bovier de, «Sur le frottemens des machines », HARS 1699, 1701, p. 104-109.

FonTENELLE B. Le Bovier de, «Sur une nouvelle proprieté de l'air, \& une nouvelle construction de Thermométre », HARS 1702, 1704, p. 1-8.

Fontenelle B. Le Bovier de, «Sur les effets du ressort de l'Air dans la Poudre à Canon, \& dans le Tonnerre », HARS 1702, 1704, p. 9-14.

FONTENELLE B. Le Bovier de, «Eloge de M. Guglielmini », HARS 1710, 1712, p. 152-166.

FontenelLe B. Le Bovier de, «Eloge de M. Poli », HARS 1714, 1716, p. 129-134.

FontenelLe B. Le Bovier de, «Eloge de M. de La Hire », HARS 1718, 1719, p. 76-89.

FONTENEAU Y., Développements précoces du concept de travail mécanique (fin 17 e s.-début $18^{e}$ s.) : quantification, optimisation et profit de l'effet des agents producteurs, Thèse de doctorat, Université Claude Bernard Lyon 1, 2011.

Formey J. H. S., "Corpusculaire », Encyclopédie ou Dictionnaire raisonné des Sciences, des Arts et des Métiers, par une Societé de Gens de Lettres, Diderot D., D’AlemberT J. Le Rond (éds.), Paris : Briasson, David, Le Breton, Durand, IV, 1754, p. 269b-270b.

FURETIERE A., Dictionnaire universel, contenant generalement tous les mots francois, tant vieux que modernes, \& les termes de toutes les Sciences \& les Arts, 3 vol., La Haye, Rotterdam : A. et R. Leers, 1690.

GABBEY A., "Newton's Mathematical Principles of Natural Philosophy : a Treatise on 'Mechanics'? », The Investigation of Difficult Things. Essays on Newton and the History of Exact Sciences, HARMAN P. M., SHAPIRO A. E. (éds.), Cambridge : Cambridge University Press, 1992, p. 305-322.

GABBEY A., "Between Ars and Philosophia Naturalis : Reflections on the Historiography of Early Modern Mechanics ", Renaissance and Revolution: Humanists, Scholars, Craftsmen and natural Philosophers in Early Modern Europe, FIELD J. V., JAMES F. A. J. L. (éds.), Cambridge, Cambridge University Press, 1993, p. 133-146.

GABBEY A., «Newton, active powers, and the mechanical philosophy », The Cambridge Companion to Newton, COHEN I. B., Sмith G. E. (éds.), Cambridge : Cambridge University Press, 2002, p. 329-357.

Galilei G., Le Mecaniche, dans Le Opere de Galileo Galilei, FAvaro A. (éd.), vol. II, Firenze : G. Barbèra, 1891.

GALILEI G., Dialogo sopra i due massimi sistemi del mondo, dans Le Opere de Galileo Galilei, FAVARO A. (éd.), vol. VII, Firenze : G. Barbèra, 1897.

GALILEI G., Discorsi e dimostrazioni matematiche intorno à due nuove scienze, dans Le Opere de Galileo Galilei, FAVARO A. (éd.), vol. VIII, Firenze : G. Barbèra, 1898.

GALILEI G., Discours concernant deux sciences nowvelles, Clavelin M. (trad.), Paris : PUF, 1995.

Galluzzi P., Momento. Studi galileiani, Rome : Edizioni dell'Ateneo \& Bizzarri, 1979.

Garber D., "Remarks on the Pre-history of the Mechanical Philosophy ", The Mechanization of natural Philosophy, GARBER D., Roux S. (éds), Dordrecht : Springer, 2012, p. 3-26.

GASSENDI P., Opera omnia, 6 vol., Lyon : L. Anisson \& I. B. Devenet, 1658.

HARRIS J., Lexicon Technicum ; Or, An Universal English Dictionary of Arts and Sciences : Explaining not only the terms of Art, but the Arts Themselves, Londres, D. Brown \& al., 1704.

HuYGENs C., Horologium oscillatorium, sive, De motv pendvlorvm ad horologia aptato demonstrationes geometrica, Paris : F. Muguet, 1673.

Huygens C., "Démonstration de l'équilibre de la balance », Divers ouvrages de Mathematiques et de Physique par Messieurs de l'Academie Royale des Sciences, Paris : Imprimerie royale, 1693, p. 313-316.

HuYgens C., Correspondance 1685-1690, dans Euvres complètes de Christiaan Huygens, t. IX, La Haye : M. Nijhoff, 1901.

HuYGENS Ch., Correspondance 1691-1695, dans CEuvres complètes de Christiaan Huygens, t. X, La Haye : M. Nijhoff, 1905.

HuYGEns Ch., Mécanique théorique et physique de 1666 à 1695, dans CEuvres complètes de Christiaan Huygens, t. XIX, La Haye : M. Nijhoff, 1937.

JANIAK A., Newton as Philosopher, Cambridge : Cambridge University Press, 2008.

JOLY B., "Chimie et mécanisme dans la nouvelle Académie royale des sciences : les débats entre Louis Lémery et Etienne-François Geoffroy », Methodos, 8, 2008.

JOLY B., Descartes et la chimie, Paris : Vrin, 2011.

L'Hospital G. E. F. marquis de, Analyse des infiniment petits, pour l'intelligence des lignes courbes, Paris, Imprimerie royale, 1696.

LA Hire Ph. de, «Défense du système de m. Descartes sur le flux et le reflux de la mer », Archives de l'académie des sciences, Pochette «Philippe de La Hire», 1692, 12 feuillets.

LA Hire Ph. de, «Traité des Epicycloïdes, et de leur usage dans les Méchaniques », Mémoires de mathématique et de physique, Paris : Imprimerie royale, 1694 , p. 1-96. 
LA Hire Ph. de, «Explication des principaux effets de la Glace, et du Froid», Mémoires de mathématique et de physique, Paris : Imprimerie royale, 1694, p. 97-213 (erreur de pagination, lire p. 97-113).

LA Hire Ph. de, "Explication des differences des sons de la Corde tenduë sur la Trompette marine », Mémoires de mathématique et de physique, Paris : Imprimerie royale, 1694, p. 213-232 (erreur de pagination, lire p. 113-132).

LA Hire Ph. de, "Dissertation des differens accidens de la Vuë», Mémoires de mathématique et de physique, Paris : Imprimerie royale, 1694, p. 233-302 (erreur de pagination, lire p. 133-202).

LA HiRE Ph. de, Mémoires de mathématique et de physique, contenant un Traité des Epicylö̈des, \& de leurs usages dans les Mechaniques. L'explication des principaux Effets de la Glace \&o du Froid. Une dissertation des Différences des sons de la Corde de la Trompette marine. Un Traité des differens Accidens de la V ûe, divisé en deux Parties, Paris : Imprimerie royale, 1694.

LA HIRE Ph. de, Traité de mécanique, ou l'on explique tout ce qui est nécessaire dans la pratique des Arts, \&o les propriétés des corps pesants lesquelles ont un plus grand usage dans la Physique, Paris : Imprimerie royale, 1695.

LA HIRE Ph. de, «Remarque sur l'usage qu'on doit faire de quelques suppositions dans la methode des infiniment petits ", Procès-Verbaux de l'Académie royale des sciences, t. XVI, séance du 23 février 1697, f. 23r-27r.

LA HIRE Ph. de, «Démonstration du temps qu'un corps employe a tomber dans une Cyclö̈de et dans ses portions, avec quelques proprietez particulieres des Cycloïdes ", Procès-Verbaux de l'Académie royale des sciences, t. XVI, séance du 8 juin 1697, f. 157v-162r.

LA HIRE Ph. de, «Regle generale pour determiner le temps que employe un corps pesant a descendre par quelque courbe que ce soit », Procès-Verbaux de l'Académie royale des sciences, t. XVII, séance du 30 août 1698, f. 368r-379v.

LA Hire Ph. de, "Experiences et Observations sur les corps qui frottent l'un contre l'autre ", Procès-Verbaux de l'Académie royale des sciences, t. XVIII, séance du 19 décembre 1699, f. 570r-573r.

LA HIRE Ph. de, "Sur la ligne Courbe que décrit un Rayon en traversant l'Atmosphére », Procès-verbaux de l'Académie royale des sciences, t. XXI, séance du 25 février 1702, f. 87r-92r

LA HiRE Ph. de, «Explication des Effets du Ressort de l'air dans les feux d'artifices et de quelques proprietez de la poudre à canon, par rapport au mouvement de la flamme du tonerre ", Procès-verbaux de l'Académie royale des sciences, t. XXI, 26 avril 1702, f. 160v-171v.

LA HiRE Ph. de, «Examen de la force de l'homme, pour mouvoir des fardeaux, tout en enlevant qu'en portant \& en tirant, laquelle est considerée absolument \& par comparaison à celle des animaux qui portent \& qui tirent, comme les chevaux », MARS 1699, 1702, p. 153-162.

LA HiRE Ph. de, «Examen de la ligne courbe, formée par un rayon de lumiere qui traverse l'Atmosphere », MARS 1702, 1704, p. 52-60.

LA HiRE Ph. de, «Suite de l'examen de la ligne courbe que décrivent les rayons de lumiere en traversant l'Atmosphere », MARS 1702, 1704, p. 182-187.

LA HIRE Ph. de, «Examen de la force necessaire pour faire mouvoir les Bateaux tant dans l'eau dormante que courante, soit avec une corde qui y est attachée \& que l'on tire, soit avec des rames, ou par le moyen de quelques machines ", MARS 1702, 1704, p. 254-280.

LA HIRE Ph. de, «Observations de l'Eclipse de Lune qui est arrivée le 11. Décembre 1704. au matin à l'Observatoire », MARS 1704, 1706, p. 352-355.

LA HIRE Ph. de, «Observations de la quantité d'eau de pluie qui est tombée à l'Observatoire pendant l'année derniere 1705, \& de la hauteur du Thermometre \& du Barometre », MARS 1706, 1707, p. 1-6.

LA HIRE Ph. de, «Sur la construction des voûtes dans les édifices », MARS 1712, 1714, p. 70-78.

LA HIRE Ph. de, «Remarques sur la cheute des corps dans l'air », MARS 1714, 1716, p. 333-344.

LA HIRE Ph. de, «Sur la hauteur de l'atmosphere », MARS 1713, 1717, p. 54-66.

LA HIRE Ph. de, «Explication de l'anneau lumineux qui paroît autour du disque de la Lune dans les Eclipses de Soleil qui sont totales ", MARS 1715, 1718, p. 161-165.

LAGRANGE J.-L., Méchanique analitique, Paris : Veuve Desaint, 1788.

Le Dictionnaire de l'Académie Françoise, 2 t., Paris : Veuve J. B. Coignard et J. B. Coignard, 1694.

LE RoY J.-B., «Dent », Encyclopédie ou Dictionnaire raisonné des Sciences, des Arts et des Métiers, par une Societé de Gens de Lettres, Diderot D., D’Alembert J. Le Rond (éds.), vol. IV, Paris : Briasson, David l'aîné, Le Breton, Durand, 1754, p. 840b$843 \mathrm{~b}$.

LEIBNIZ G. W., Sämtliche Schriften und Briefe, Reihe III, Band 6 (Jan. 1694-Juni 1696), Berlin : Akademie-Verlag, 2004. LEIBNIZ G. W., Sämtliche Schriften und Briefe, Reihe III, Band 7 (Juli 1696-Dez. 1698), Berlin : Akademie-Verlag, 2011. LEIBNIZ G. W., Sämtliche Schriften und Briefe, Reihe I, Band 24 (Aug. 1705-Apr. 1706), Berlin : Akademie-Verlag, 2017. LEIBNIZ G. W., Sämtliche Schriften und Briefe, Reihe III, Band 9 (Jan. 1702-Sept. 1705), Berlin : Akademie-Verlag (https://leibnizedition.de/reihen/reihe-iii/ consulté le 12/02/2019), (à paraitre).

LEMERY N., «Explication Physique \& Chymique des Feux souterrains, des Tremblemens de Terre, des Ouragans, des Eclairs \& du Tonnerre », MARS 1700,1703, p. 101-110.

MAIRAN J. J. D. de, Dissertation sur la Glace ou explication physique de la formation de la Glace, \& de ses divers Phénomenes, qui a remporté le prix à l'Academie Royale des belles Lettres, Sciences \& Arts de Bordeaux, pour 1716, Bordeaux, R. Brun, 1716 (2e éd. en 1717 ; 4 éd. en 1749).

MAncuso P., "The Metaphysics of the Calculus : A Foundational Debate in the Paris Academy of Sciences, 1700$1706 »$, Historia Mathematica, vol. 16, 1989, p. 224-248.

MARIOTTE E., Traitté de la percussion ou chocq des corps, Paris : E. Michallet, 1673. 
Mariotte E., Essays de physique. Second Essay. De la nature de l'Air, Paris : E. Michallet, 1679.

Mariotte E., Essays de physique. Troisieme Essay. Du chaud et du froid, Paris : E. Michallet, 1679.

MARIOTTE, Expériences touchant les couleurs et la congélation de l'eau, dans CEuvres de Mr. Mariotte, t. 2, Leyde : P. Vander, 1717.

MCGUIRE J. E., « Boyle's Conception of Nature », Journal for the History of Ideas, 33/4, 1972, p. 523-542.

METZGER H., Les doctrines chimiques en France du début du XVII à la fin du XVIII siècle, Paris : PUF, 1923.

Molieres J. P. de, Leçons de Phisique, 4 t. Paris : Veuve Brocas, Musier, Billot, 1734-1739.

Newton I., Philosophice Naturalis Principia Mathematica, Londres : Streater, 1687.

NOLLET J. A., Leçons de physique expérimentale, t. I, Paris : Frères Guérin, 1743.

Nouveau Dictionnaire de l'Académie Françoise, 2 t., Paris : J. B. Coignard, 1718.

PALMIERI P., "Breaking the circle : the emergence of Archimedean mechanics in the late Renaissance ", Archives for History of Exact Sciences, 62/3, 2008, p. 301-346.

PARENT A., «Sur la plus grande perfection possible des Machines », MARS 1704, 1706, p. 232-338.

PARENT A., « Nouvelle Statique avec frotemens \& sans frotemens, ou Regles pour calculer les frotemens des Machines dans l'état d'équilibre. Premier Mémoire, qui contient tout ce qui se fait sur des plans inclinés ", MARS 1704, 1706, p. 173-186.

PARENT A., «Second Mémoire. Trouver la force avec laquelle il faut pousser un coin, pour séparer un corps ou directement, ou sur un point fixe, ou sur les deux », MARS 1704, 1706, p. 186-197.

PARENT A., «Troisieme Mémoire. Des Poulies \& de leurs Tourillons », MARS 1704, 1706, p. 206-209.

PARENT A., «Quatiéme Memoire de la nouvelle Statique avec Frottemens \& sans Frottemens : Suite des Memoires de 1704. Calcul des Puissances necessaires pour vaincre les Frottemens des Essieux dans les Machines, \& des angles que leurs directions doivent faire, afin que ces Frottemens soient les moindres qu'il se puisse », MARS 1712, 1714, p. 96107.

Pestre J., D’Alembert J. Le Rond, «Cartésianisme », Encyclopédie ou Dictionnaire raisonné des Sciences, des Arts et des Métiers, par une Societé de Gens de Lettres, Diderot D., D’AlEmbert J. Le Rond (éds.), Paris : Briasson, Le Breton, Durand, t. II, 1752, p. 716a-726a.

"Pignon », Encyclopédie ou Dictionnaire raisonné des Sciences, des Arts et des Métiers, par une Société de Gens de Lettres, mis en ordre et publié par M ${ }^{\mathrm{r} .} * * *$, vol. XII, Neufchastel : S. Faulche \& Compagnie, 1765, p. 615b-616b.

Picolet G., «Annexe IV. La Hire entre sciences et architecture : une chronologie », Philippe de La Hire 1640-1718. Entre architecture et sciences, Becchi A., Rousteau-Chambon H., SAKArovitch J. (éds.), Paris : A. et J. Picard, 2013, p. 261-296.

Poisson N., Traité de la méchanique, composé par Monsieur Descartes, Paris : C. Angot, 1668.

Procès-verbaux de l'Académie royale des sciences, t. III, 1668.

Procès-verbaux de l'Académie royale des sciences, t. XX, 1701.

Procès-verbaux de l'Académie royale des sciences, t. XXIV, 1705.

Radelet-De-Grave P., "La mécanique », Philippe de La Hire 1640-1718. Entre architecture et sciences, BECCHI A., Rousteau-Chambon H., Sakarovitch J. (éds.), Paris : A. et J. Picard, 2013, p. 159-175.

RATTE E. H. de, «Froid», Encyclopédie ou Dictionnaire raisonné des Sciences, des Arts et des Métiers, par une Societé de Gens de Lettres, Diderot D., D’Alembert J. Le Rond (éds.), Paris : Briasson, David, Le Breton, Durand, t. VII, 1757, p. 312a319b.

RatTe E. H. de, "Glace », Encyclopédie on Dictionnaire raisonné des Sciences, des Arts et des Métiers, par une Societé de Gens de Lettres, Diderot D., D’Alembert J. Le Rond (éds.), Paris : Briasson, David, Le Breton, Durand, t. VII, 1757, p. 677a684b.

REgIS P.-S., Systême de Philosophie, contenant la Logique, Metaphysique \& Morale, 7 t. Lyon : Anisson, Posuel \& Rigaud, 1691. Rohault J., Traité de physique, Paris : Veuve de Charles Savreux, 1671.

Roux S., La philosophie mécanique (1630-1690), Thèse de Doctorat, EHESS Paris, 1996.

Roux S., «Le scepticisme et les hypothèses de la physique », Revue de Synthèse, 2-3, 1998, p. 211-255.

Roux S., "La philosophie mécanique de Boyle», L'atomisme aux XVIIe et XVIII siècles, SALEM J. (éd.), Paris : Publications de la Sorbonne, 2000, p. 119-133.

Roux S., "Cartesian Mechanics », The Reception of the Galilean Science of Motion in Seventeenth-Century Europe, PALMERINO C. R., THijssen J. M. M. H. (éds.), Dordrecht : Springer, 2004.

Roux S., "La philosophie naturelle à l'époque de Le Nôtre. Remarques sur la philosophie mécanique et sur le cartésianisme », Fragments d'un paysage culturel, André Le Nôtre, Institutions, arts, sciences et techniques, FAHRAT G. (éd.), Sceaux : Musée de l'Ile de France, 2006, p. 98-116.

SCHмIт Chr., "Rapports entre équilibre et dynamique au tournant des $17^{\mathrm{e}}$ et $18^{\mathrm{e}}$ siècles », Early Science and Medecine, 19/4, 2014, p. 505-548.

SCHMIT Chr., « Méchanique, statique, dynamique. Répartition du savoir et définitions dans l'Encyclopédie », 1ère partie, Recherches sur Diderot et sur l'Encyclopédie, 49, 2014, p. 225-256 ; 2 ${ }^{\mathrm{e}}$ partie, ibid., 50, 2015, p. 274-299.

SCHMIT Chr., «Les quatre prix académiques remportés par Lozeran du Fesc », Autour de Descartes et Newton. Le paysage scientifique lyonnais dans le premier XVIII siècle, CREPEL P., SCHMIT Chr. (éds.), Paris : Hermann, 2017, p. 193-224. 
SCHMIT Chr., "Les dissertations sur la calcination, le magnétisme et l'électricité du père Béraud (1747, 1748, 1755)», Autour de Descartes et Newton. Le paysage scientifique lyonnais dans le premier XVIII siècle, CREPEL P., SCHMIT Chr. (éds.), Paris : Hermann, 2017, p. 273-322.

SCHмIT Chr., La philosophie naturelle de Malebranche au XVIII siècle. Inertie, causalité, petits tourbillons, Paris : Classiques Garnier, 2020.

SERIS J.-P., Machines et communication, du théâtre des machines à la mécanique industrielle, Paris : Vrin, 1987.

VARIGNON P., Projet d'une nouvelle mechanique avec un Examen de l'opinion de M. Borelli, sur les propriétez. des Poids suspendus par des Cordes, Paris : Veuve d'Edme Martin, J. Boudot, E. Martin, 1687.

VARIGNON P., « Nouvelle Demonstration des mouvemens Isochrones dans la Cyclö̈de renversée », Procès-verbaux de l'Académie royale des sciences, t. XVI, séance du $1^{\text {er }}$ juin, 1697, f. 151v-153v.

VARIGNON P., «Seconde et Troisiéme Demonstration des mouvemens Isochrones dans la Cycloïde renversée », Procèsverbaux de l'Académie royale des sciences, t. XVI, séance du 8 juin, 1697, f. 162r-165r.

VARIGNON P., «Nouvelle Remarque sur les mouvemens Isochrones dans la Cycloïde renversée », Procès-verbaux de l'Académie royale des sciences, t. XVI, séance du 15 juin, 1697, f. 171r-172v.

VARIGNON P., «Manière generale de trouver les courbes Isochrones pour toutes les hipotheses imaginables d'acceleration dans les corps qui tombent », Procès-verbaux de l'Académie royale des sciences, t. XVII, séance 4 janvier, 1698, f. $64 \mathrm{v}-68 \mathrm{r}$.

VARIGNON P., « Regle generale pour toutes sortes de mouvement de vitesses quelconques variées a discretion », Procèsverbaux de l'Académie royale des sciences, t. XVII, séance du 5 juillet, 1698, f. 297v-305r.

VARIGNON P., «Application de la Regle generale des vitesses variées, comme on voudra aux mouvemens pour toutes sortes de courbes, tant mecaniques que geometrique. D'où l'on deduit encore une nouvelle manière de démontrer les chuttes isochrones dans la Cycloïde renversée », Procès-verbaux de l'Académie royale des sciences, t. XVII, séance du 6 septembre, 1698, f. 387r-391v.

VILAIN Chr., «La question du centre d'oscillation de 1660 à $1690 »$, Physis, 37, 2000, p. 21-51.

VILAIN Chr., «La question du centre d'oscillation de 1703 à $1743 »$, Physis, 37, 2000, p. 439-466.

WEBSTER C., "The discovery of Boyle's law and the concept of the elasticity of air in the seventeenth century ", Archive for History of Exact Sciences, 2, 1965, p. 441-502.

WestFall R. S., Force in Newton's physics. The Science of Dynamics in the Seventeenth Century, Londres, New York: MacDonald, Elsevier, 1971. 